\title{
The U.S. Geological Survey Coal Quality (COALOUAL) Database Version 3.0
}

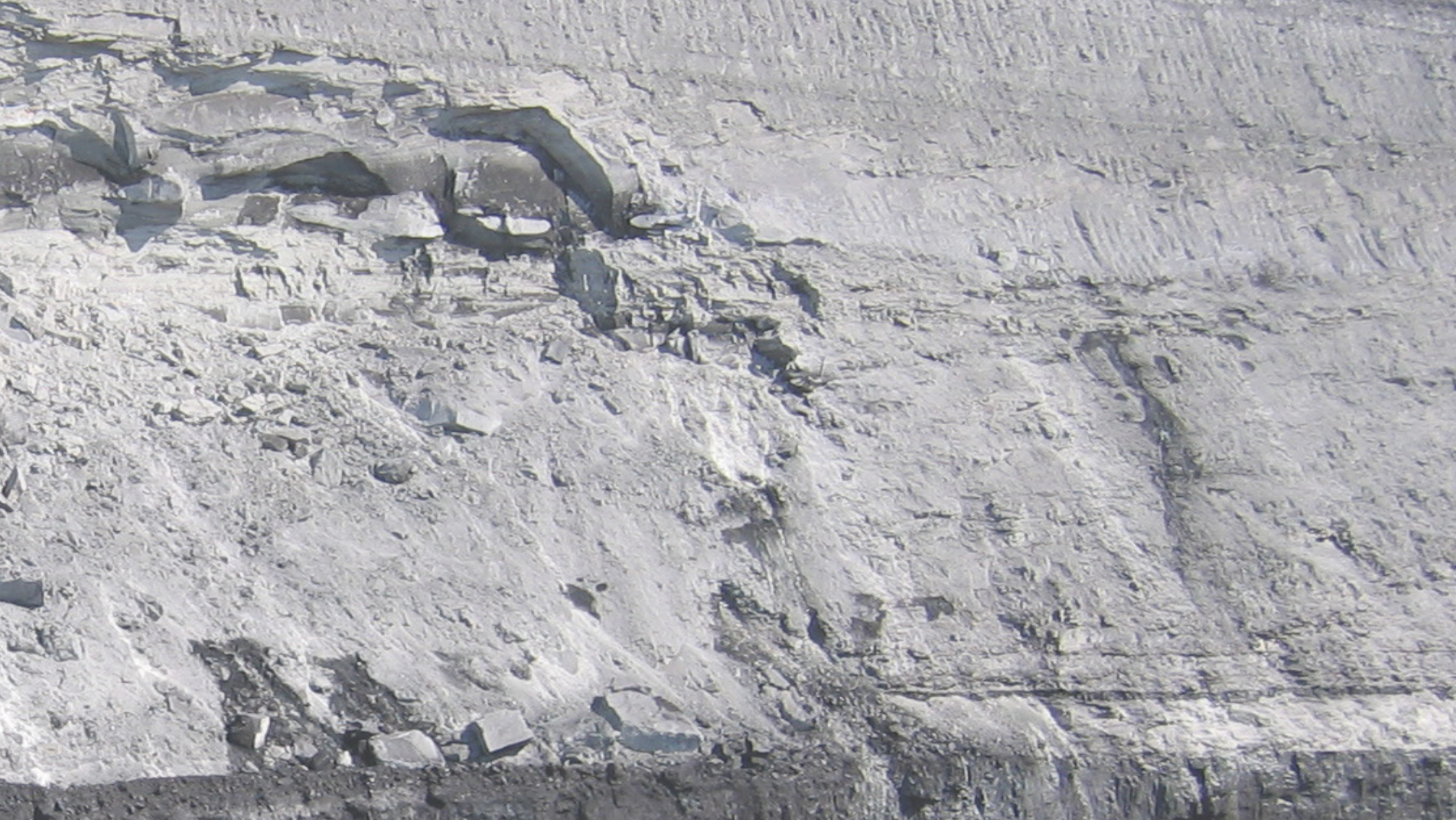




\section{The U.S. Geological Survey Coal Quality (COALOUAL) Database Version 3.0}

By Curtis A. Palmer, Charles L. Oman, Andy J. Park, and James A. Luppens

Data Series 975

U.S. Department of the Interior

U.S. Geological Survey 


\section{U.S. Department of the Interior SALLY JEWELL, Secretary}

\section{U.S. Geological Survey Suzette M. Kimball, Director}

\section{U.S. Geological Survey, Reston, Virginia: 2015}

For more information on the USGS - the Federal source for science about the Earth, its natural and living resources, natural hazards, and the environment—visit http://www.usgs.gov or call 1-888-ASK-USGS.

For an overview of USGS information products, including maps, imagery, and publications, visit http://www.usgs.gov/pubprod/.

Any use of trade, firm, or product names is for descriptive purposes only and does not imply endorsement by the U.S. Government.

Although this information product, for the most part, is in the public domain, it also may contain copyrighted materials as noted in the text. Permission to reproduce copyrighted items must be secured from the copyright owner.

Suggested citation:

Palmer, C.A., Oman, C.L., Park, A.J., and Luppens, J.A., 2015, The U.S. Geological Survey coal quality (COALOUAL) database version 3.0: U.S. Geological Survey Data Series 975, 43 p. with appendixes, http://dx.doi.org/10.3133/ ds975.

ISSN 2327-638X (online)

Cover: Photo from the Wyoming Powder River Basin. (Photo by David Scott, U.S. Geological Survey, Denver, Colo.) 


\section{Contents}

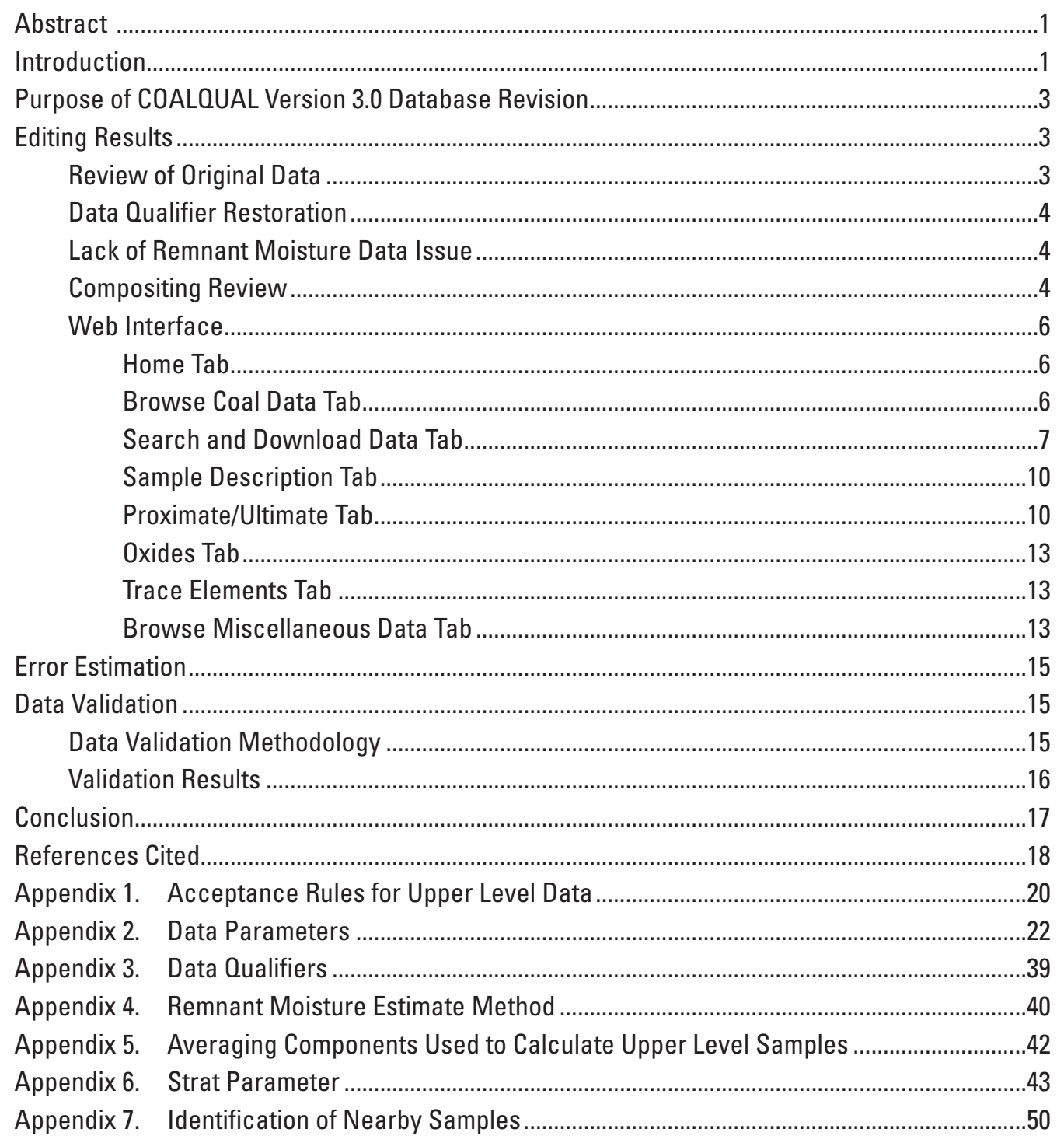




\section{Figures}

1. A screen image of the COALOUAL Version 3.0 web interface banner and tabs................6

2. A screen image of the COALOUAL Version 3.0 Home tab.................................................

3. An example of the COALQUAL Version 3.0 Browse Coal Data tab and search screen ...7

4. An example of coal samples that are from either the same location, or located at the same mine or power plant (within one mile distance)...............................................8

5. An example of the relationship of coal subsamples to an Upper Level sample ................8

6. An example of the COALOUAL Version 3.0 coal Sample Detail page ..............................9

7. A screen image of the COALOUAL Version 3.0 Search and Download Data tab .............9

8. The pull down menu for report types on the Search and Download Data tab ................9

9. The Report Format pull down menu on the Search and Download Data tab ...................9

10. Examples of two CSV files showing Upper Level samples and related subsamples ......10

11. An example of search results filtered by search criteria .............................................11

12. A screen image of the radio buttons for options to search coal or miscellaneous samples....

13. A screen image of the pull down menu for the $L$ and $G$ data qualifiers ........................11

14. A screen image of the check box and button for search criteria .................................11

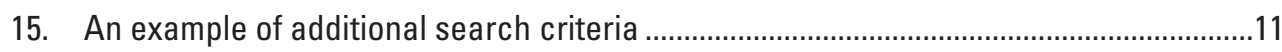

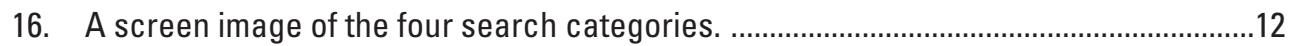

17. The search criteria in the Sample Description tab ......................................................12

18. The search criteria in the Proximate/Ultimate tab.......................................................12

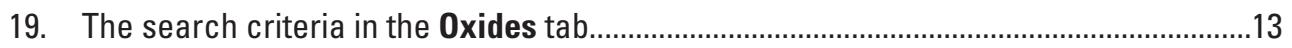

20. The search criteria in the Trace element tab ……......................................................13

21. A screen image of the Browse Miscellaneous Data tab .................................................14

22. A detailed example of miscellaneous data .......................................................................14

\section{Tables}

1. Validation summary for the degree of agreement between the predicted higher heating value and the ultimate and measured HHV analyses

2. Validation summary for the degree of agreement between the proximate analyses and the carbon and hydrogen values from the ultimate analyses .................................17

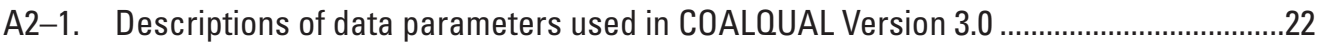

A3-1. Descriptions of data qualifiers used in COALOUAL Version. 3.0 ...................................39

A4-1. Input and criteria for estimating remnant moisture...................................................40

A6-1. A simple example of a stratigraphic sequence with two coal benches, and an Upper Level sample mathematically composited from the two coal bench samples.....43

A6-2. A list of additional letter designations used for non-coal material associated with coal bed and coal materials.

A6-3. An example of a location with a coal sequence with roof, parting, and floor samples

A6-4. An example of a location with a miscellaneous rock sample and a coal sequence in two different stratigraphic sequences. 
A6-5. An example of three stratigraphic sequences that are at the same location, or so similar in location there is no apparent difference in latitude and longitude...................46

A6-6. An example of a location with a stratigraphic sequence that contains both a duplicate and a floor sample. . .46

A6-7. An example of a location with ASTM composite data and three bench samples without ASTM data.

A6-8. An example of a stratigraphic sequence at a location with U.S. Geological Survey (USGS) composite data and two bench samples without USGS data

A6-9. An example of a location with an Upper Level physically composited sample using both ASTM and U.S. Geological Survey data, a roof, three coal benches, and a floor

A6-10. An example of a location with two single Upper Level samples with splits treated with alkaline solution ..... . .48

A6-11. An example of a location with an informational sample that contains a parting ............48

A6-12. An example of a location with a stratigraphic sequence with a parting removed and analyzed separately 


\title{
The U.S. Geological Survey Coal Quality (COALOUAL) Database Version 3.0
}

\author{
By Curtis A. Palmer', Charles L. Oman', Andy J. Park', and James A. Luppens ${ }^{2}$
}

\section{Abstract}

Coal has often been described as one of the most difficult materials to sample because of its inherent heterogeneity. Therefore, fundamental to any coal assessment is an understanding of the impacts of the geological heterogeneity on coal quality variability for a given area.

Since the mid-1970s, the U.S. Geological Survey (USGS) has maintained a coal quality database of national scope named USCHEM, which currently contains data for over 13,000 samples. A subset of the USCHEM database called COALQUAL Version 1.3 was initially published in 1994 . Version 3.0 of the COALQUAL database represents a major editing effort to resolve some of the DOS software limitations used by earlier versions of the database.

Because of database size limits during the development of COALQUAL Version 1.3, many analyses of individual bench samples were merged into whole coal bed averages. The methodology for making these composite intervals was not consistent. Size limits also restricted the amount of georeferencing information and forced removal of qualifier notations such as "less than detection limit" $(<)$ information, which can cause problems in using the data. A review of the original data sheets revealed that COALQUAL Version 2.0 was missing information, which was needed for a complete understanding of a coal section. Another important database issue to resolve was the USGS "remnant moisture" problem. Prior to 1998, tests for remnant moisture (as-determined moisture in the sample at the time of analysis) were not performed on any USGS major, minor, or trace-element coal analyses. Without the remnant moisture, it is impossible to convert the analyses to a usable basis (as-received, dry, etc.). Based on remnant moisture analyses of hundreds of samples of different ranks and known residual moisture reported (moisture reported at the time of the ultimate and proximate analysis) after 1998, it was possible to develop a method to provide reasonable estimates of remnant moisture for older data to make it more useful in COALQUAL Version 3.0. In addition, the COALQUAL Version 3.0 database is improved by (1) adding qualifiers, including statistical programming to deal with the qualifiers; (2) clarifying the sample compositing problems; and (3) adding associated samples (discussed in more detail in report). Version 3.0 of COALQUAL also represents the first attempt to incorporate data verification by mathematically crosschecking certain analytical parameters. Finally, a new database system was designed and implemented to replace the outdated DOS program used in earlier versions of the database. The COALQUAL Version 3.0 database is located at http://ncrdspublic.er.usgs.gov/coalqual/.

\section{Introduction}

Coal has often been described as one of the most difficult materials to sample because of its inherent heterogeneity (Luppens and others, 1992). Fundamental to any coal assessment is an understanding of the impacts of the geological heterogeneity on coal quality variability for a given area. Consequently, a coal bed may often be subsampled in several intervals (benches) rather than a single sample of the entire bed to document vertical quality variations at a sampling location.

Since the mid-1970s, the U.S. Geological Survey (USGS) has maintained a coal quality database of national scope named USCHEM, which currently contains data for over 13,000 samples. A subset of the USGS coal quality database USCHEM called COALQUAL Version 1.3 was first released in 1994 (Bragg and others, 1994). COALQUAL Version 2.0 (Bragg and others, 1997) was released in 1997 without changes to the data, to accommodate the Microsoft XP operating system. The first two releases of COALQUAL provided public data from almost 7,500 coal and coal-related samples or composites analyzed from the early 1970s until 1990.

${ }^{1}$ U.S. Geological Survey, Reston, VA

${ }^{2}$ U.S. Geological Survey, Denver, CO 
The U.S. Geological Survey Coal Quality (COALOUAL) Database Version 3.0

The USCHEM database was not published in its original format for several reasons, including the following:

- USCHEM data were stored on an as-determined basis.

- Some elements and (or) oxides were analyzed on a whole-coal basis, while others were analyzed on the coal ash and maintained in multiple basis types as originally reported. If all data were not converted to the same basis, the data could be misinterpreted or misused by those unfamiliar with coal chemical analyses. In COALQUAL Version 3.0, all elemental data for samples are converted to a dry, whole-coal basis.

- The most important analyses reported in COALQUAL Version 3.0 are from samples that represent the entire coal bed at the point of sampling. Many of the coal samples were originally collected and analyzed as a series of individual benches, particularly for the thick coal beds from the western United States. The whole- or full-bed value for each parameter was determined from the weighted mathematical composite of the bench analyses based on sample interval thicknesses. For example, for a 9-foot (ft) sample with two benches, one 3-feet thick and one 6-feet thick, with concentrations of iron ( $\mathrm{Fe})$ of 1.0 milligram per kilogram $(\mathrm{mg} / \mathrm{Kg})$ and $1.5 \mathrm{mg} / \mathrm{Kg}$ respectively, the concentration of Fe for the full-bed is 1.33 as follows:

$$
(3 \mathrm{ft} / 9 \mathrm{ft} \times 1.0 \mathrm{mg} / \mathrm{Kg})+(6 \mathrm{ft} / 9 \mathrm{ft} \times 1.5 \mathrm{mg} / \mathrm{Kg})=1.33 \mathrm{mg} / \mathrm{Kg}
$$

- In a few cases, samples were physically composited by combining properly prepared samples of each bench in the weight ratio of their thicknesses. While this method is only approximate, as differences in the densities of the benches would lead to small differences between mathematical and physical composites, we found that for samples where both types of composites were available the data were similar and we treated them as duplicates. The original USCHEM database also lacked mathematical composite data, as well as data on coal weathering and identification of duplicates. Those conditions made it difficult, and in some cases, impossible to determine chemical data for non-weathered full-bed samples, which may have originated from multiple samples and (or) samples that may have experienced various degrees of weathering. It should be emphasized that only non-weathered, full-bed (Upper Level) coal samples should be used for most coal resource studies. A total of 7,657 Upper Level samples are included in COALQUAL Version 3.0.

In addition to non-weathered, full-bed coal samples (Upper Level samples), USCHEM contained data for individual coal benches, which were combined to form Upper Level samples and data for partings, roof, floor, and miscellaneous coal and coalrelated samples. These miscellaneous samples included coal byproducts, fly ash, bottom ash, chemically treated coal samples, rock samples related to the coal stratigraphy, tonsteins, or non-representative samples from partial-bed or coal mining efforts. Appendix 1 provides a complete list these miscellaneous samples.

Prior to 1998, as-determined moisture analyses (moisture in the sample at the time of analysis or remnant moisture) were not performed routinely on USGS coal samples analyzed for major, minor, or trace elements. Originally, it was felt that differences in moisture content of the dried samples were less significant than errors in trace element determinations. Therefore, all analytical values were reported on a remnant moisture basis. However, because the remnant moisture values of the samples were not measured, conversions to any other analytical basis (as-received, dry, or dry ash-free) were impossible. This is a significant limitation to the use of the data in previous versions of COALQUAL as many users often needed analytical results reported on either an as-received or a dry basis. Additionally, qualifying (censored) data such as (1) values below the detection limit, (2) upper limits caused by non-linear standards, (3) values not determined because of spectral interferences, and (4) values not determined for other reasons, were stored in the USCHEM database. If a parameter was not analyzed, a "zero" with a "B" qualifier notation was used.

When the original COALQUAL Version 1.3 database was being compiled, potential users were faced with limited computer memory, storage capacity, and media availability, as well as minimal broadband capacity. In 1994, the USCHEM database including appropriate metadata could not fit on a single CD-ROM disk. These limitations required a number of data storage compromises while compiling the COALQUAL Version 1.3 data, when database functionality relied on the DOS-based operating system. The COALQUAL Version 2.0 database revision utilized the Microsoft Windows XP operating system, but the database remained unchanged. COALQUAL Version 3.0 has been updated to include all supportive metadata, as well as revised data values as warranted during the extensive editing process.

In earlier versions of COALQUAL, analyses of individual bench samples were merged into full-bed averages for each location, even though the composited bench samples did not represent the entire bed or the mined coal in some cases. Additionally, size limitations in the Microsoft Excel spreadsheets (mainly the number of columns available in earlier COALQUAL versions) restricted the amount of georeferencing information and forced removal of qualifier notations such as "less than detection limit" $(<)$ information. Furthermore, most partings were excluded as were bench-sample data, which prevented compilation into a full-bed sample. Although impure coal is defined as having dry ash content between 25 to 50 percent by American Society for Testing and Materials International (ASTM) Standard D121-09 (ASTM, 2014), all coal samples in USCHEM whose USGS and ASTM ash 
values on an as-received basis were greater than 33 percent, were not included in COALQUAL Version 1.3 and Version 2.0 (Wood and others, 1983). These samples are incorporated into the COALQUAL Version 3.0 database.

\section{Purpose of COALQUAL Version 3.0 Database Revision}

An extensive revision of the COALQUAL database was initiated to take advantage of advances in computer technology and software design since the original versions of COALQUAL were developed. This growth in technology resolved the original compromises due to limited memory and storage capacity and also provided improved database design and an improved webbased delivery platform. The primary objectives for this revision were as follows:

1. Complete a thorough audit of the COALQUAL analytical data versus original hard copy analytical sheets (and other USGS databases) and make appropriate corrections.

2. Resolve the forced removal of data qualifier notations such as "less than detection limit" (L) by referring back to the original hardcopy analytical sheets, and resolve the problems associated with the use of "zeros" for parameters that were not analyzed.

3. Investigate and remedy the problem of missing USGS remnant moisture values.

4. Review the methodology and the consistency for compositing bench samples and add associated samples such as roof, floor, and parting analyses.

5. Revise the COALQUAL georeferencing information based on original hard copy analytical sheets and make necessary corrections and enhancements.

6. Investigate the feasibility of estimating errors associated with combining bench samples into full-bed data and estimating moisture values.

7. Investigate the viability of developing data validation tools.

8. Develop an improved web-based interface for database access.

\section{Editing Results}

The comprehensive editing effort of the COALQUAL Version 3.0 database resulted in a significant improvement, not only in the accuracy of the data, but also resulted in an improved user interface for COALQUAL Version 3.0. Additionally, several significant limitations for use of the data were also addressed.

\section{Review of Original Data}

In order to ensure that COALQUAL Version 3.0 was accurate and complete, the review started with the original data from USCHEM. The analytical data in USCHEM were extensively checked before previous versions of COALQUAL were released. For this new version, all of the stratigraphic, geologic, and geospatial data were checked with original paper copies of the analytical reports and sample description sheets. Additional columns of data were added to include all pertinent data available for each sample.

Table A2 -1 of appendix 2 provides a list and brief description of all data parameters. Those parameters added to COALQUAL Version 3.0 are highlighted with asterisks and bold lettering in table A2-1. The new parameters include (1) estimated remnant moisture; (2) USGS ash on a dry basis; (3) several columns related to major coal-forming elements; (4) data verification classifications for ultimate and proximate analysis; (5) carbonate carbon, organic carbon, and total carbon; and (6) columns related to rank of coal (ASTM apparent rank, British thermal units per pound (Btu/lb), volatile matter, and fixed carbon; all on a moist, mineralmatter-free basis). Additional columns document stratigraphic relationships between samples and major ash-related elements that were originally reported only as oxides (are now also reported as elements). 
The U.S. Geological Survey Coal Quality (COALOUAL) Database Version 3.0

\section{Data Qualifier Restoration}

Qualifier characters for censored data are defined in appendix 3. In older versions of COALQUAL, no qualifiers were reported. Data values below the detection limit were presented as 0.7 times the reported detection limit if the "L" (less than) data qualifier was used, or as " 0 " if the " $N$ " (not detected) or " $\mathrm{B}$ " (no data) qualifier was used (appendix 3). When a "G" (greater than) qualifier was present, it was ignored and the data value was retained. The total number of qualified data values was reported as a percent of the total number of samples in the technical information metadata for COALQUAL Versions 1.3 and 2.0, but it was not possible to determine what individual values were qualified. In COALQUAL Version 3.0, qualifiers from USCHEM were retained and new qualifiers were added based on hardcopies of lab results and USGS and State Geological Survey publications.

The use of "zeros" was also problematic in older versions of COALQUAL. If a parameter was not analyzed, a " 0 " with a "B" qualifier was used. Unfortunately, some parameters like the free-swelling index, loss on ignition (LOI), and residual moisture have valid zero values making it impossible to distinguish between real zero values and "not analyzed" values or those with "N" qualifiers. The problems associated with the use of zeros were resolved by creating the more extensive list of data qualifiers in appendix 3.

\section{Lack of Remnant Moisture Data Issue}

The initial steps in the analysis of a coal sample is pulverizing the as-received samples to minus 8 mesh and air-drying the samples per ASTM D3302 (ASTM, 2014). The purpose of the air-drying is to allow the samples to reach equilibrium with laboratory ambient temperature and humidity. This equilibrium condition minimizes sample weight gains or losses during the subsequent handling, weighing, and analyses of the coal. The moisture content of the air-dried samples prepared for ultimate and proximate analysis is termed "residual moisture." The moisture content in analytical samples can change when the samples are exposed to an atmosphere in which the relative humidity differs from that prevailing during sample preparation. Consequently, it is advisable to measure moisture at the same time an analytical property is determined (ASTM, 2014; see ASTM D3302). Without a moisture value, the calculation of a coal analysis from an as-determined to different bases (as-received, dry, and so forth) cannot be accurately accomplished (ASTM, 2014; see ASTM D3180).

Unfortunately, although residual moistures were determined at the time of ultimate and proximate analysis, remnant moisture was not determined for all samples in the USCHEM database at the time of the major, minor, and trace element analyses. COALQUAL Version 3.0 addresses missing values for the remnant moisture content. Ultimate and proximate analyses generally included residual moisture values. The residual moisture values were usually determined in contract laboratories generally 6 to 20 months before elemental data were generated at the USGS laboratories located near Washington, D.C. (in Reston, Va.) or near Denver, Colorado (in Lakewood). The altitude and humidity of most contract lab locations is similar to Washington, D.C., but is significantly different than that in Denver, Colorado. The altitude and the drier climate in Denver promoted the possibility of further sample drying even if sealed in polyethylene containers. Samples that were not tightly sealed may have experienced accelerated drying. Higher-rank coal samples inherently have much lower residual moisture contents, and therefore are typically less susceptible to significant moisture loss during storage. Thus, the rates of moisture loss and (or) gain is different in Denver versus Washington D.C, especially for low-rank, high-moisture coals.

After 1998, remnant moisture was determined at the time of analysis on a large number of samples with various ranks and compared to the respective residual moisture contents determined on air-dried samples at the time of proximate and ultimate coal analyses. From these comparisons, a methodology was developed to derive estimates for the remnant moisture content in the USGS laboratory samples using moisture data from samples with known residual moisture and (or) air dried-loss values and coal rank.

Major and trace element data on a dry, whole-coal basis are estimated using procedures that are described in appendix 4. Samples with estimated remnant moisture are qualified with the letter " $r$ " (appendix 3). Procedures for estimating the remnant moisture content (for samples without air-dried loss, or residual moisture information that are based on the apparent rank only) are also described in appendix 4 and qualified with the letter "e" (appendix 3). The estimates for moisture for samples qualified with the letter "e" assume all samples of a given rank have the same moisture value, and therefore are not generally as accurate as those samples qualified with an " $r$ " (appendix 3). Estimated moisture contents, as well as the as-determined data values, are listed in the COALQUAL Version 3.0 database. Appendix 4 describes the method developed, which was designed to minimize the errors resulting from using these estimated moisture values to calculate elemental concentrations to a dry basis.

\section{Compositing Review}

Coal data for a given location are typically most useful if the sample(s) are reported on a whole- or full-bed basis. Partial-bed samples provide some information, but are not representative of the full-bed at the point of sampling. Therefore, every attempt 
to provide as much data in COALQUAL Version 3.0 on a full-bed basis (Upper Level samples) was made. Often, coal beds are sampled in two or more benches or layers to better characterize vertical variations in coal quality. The individual benches are then composited, either mathematically or physically, to provide weighted average values for coal quality parameters on a full-bed basis. COALQUAL Version 3.0 has correctly composited data to completely represent a coal bed (referred to as a "full" coal bed) like previous versions, which includes full-channel samples, core samples, and composited samples.

Most of the data for full coal beds are identical to the data in COALQUAL Version 1.3 and Version 2.0, but calculated to a dry basis for elemental data. All elements reported on a remnant moisture, whole-coal basis in the previous versions of COALQUAL are reported on a dry, whole-coal basis in COALQUAL Version 3.0. The ultimate and proximate data are on an as-received basis in all versions of COALQUAL. However, in COALQUAL Version 1.3 and Version 2.0, hydrogen $(\mathrm{H})$ and oxygen $(\mathrm{O})$ values included $\mathrm{H}$ and $\mathrm{O}$ concentrations in residual moisture, which is not consistent with coal industry practices. In some cases, full-beds were constructed the same way for all versions of COALQUAL. Each version provides information on how the full-beds were constructed. A set of rules for Version 3.0 is included in appendix 1, Acceptance Rules for Upper Level Data. A few samples in the original COALQUAL Version 1.3 were not included in this version because they did not meet the standards required, and a few new samples were added. COALQUAL Version 3.0 provides criteria for rejecting samples not included in the set of full-bed samples.

The 15,121 individual samples in this database are divided into several groups based on categories of full-bed samples and sample type. The acceptance rules for compositing bench samples into full-bed samples are provided in appendix 1. Samples in the database are organized on several classifications or levels as follows:

1. Upper Level-Samples represent unweathered, full coal bed intervals at a given location. These full-bed samples are the basis of COALQUAL Version 3.0, which is consistent with previous versions of COALQUAL. The new version of COALQUAL Version 3.0 has 7,657 full-bed samples. These samples are either single full-bed samples, or may be represented by the mathematical average of a full-bed interval sampled in multiple benches. Second Level data for multiple benches (duplicate and (or) composited samples), are composited to represent a single Upper Level sample using guidelines found in appendix 5 .

2. Second Level —-Individual coal samples that are composited to represent a single Upper Level sample including:
a. Bench samples (either drill core or face-channel samples).
b. Duplicates (provided as a measure of reliability).
c. Physical composites of partial-beds (or full-beds, if used in combination with a. and (or) b. above to determine an Upper Level sample).

3. Third Level—All samples collected at the location of the Upper Level sample.

b. Non-coal samples such as roof, floor, and partings, and other miscellaneous samples (see appendix 1).

4. Fourth Level-Samples that are similar to Third Level samples except they are within a limited geographic area of the Upper Level sample, but not at the same location. It should be noted that Upper Level, Second Level, and Third Level samples may be Fourth Level samples at nearby locations. Fourth Level samples are from:
a. The same mine.
b. Within a mile of the location of Upper Level samples.

5. Fifth Level-Miscellaneous samples not related to full-bed samples.

The first three levels contain samples from a single latitude and longitude location. These samples are (1) full-bed samples, (2) benches that make up full- or partial-bed samples, and (3) non-coal samples such as roof, floor, and partings at different vertical locations. Most samples do not have exact vertical locations (depth from surface), but nearly all samples have known relative vertical locations. For the few samples with unknown relative locations, those samples have been identified by using slightly different longitude or latitude coordinates a few feet from the original location. The relative vertical stratigraphic positions are given by the Strat parameter, which is defined in appendix 2 and shown as *Strat* in table A2-1. Appendix 6 also provides detailed information about the Strat parameter. 


\section{Web Interface}

In addition to including new data in Version 3.0, a new web interface was created because the additional data and revised format required these changes, and technological advances enabled more efficient presentation of the data. This new interface makes it possible to present full-bed sample data (Upper Level samples) as in previous versions of COALQUAL (Version 1.3 and Version 2.0), as well as all data used to calculate the full-bed data including duplicate samples and benches. The data from USCHEM that were not available in the earlier versions of COALQUAL, such as roof, floor, and parting samples can also be accessed from the Upper Level samples in the database. Georeferencing efforts to identify other samples located within one mile of a sample's location, is computed by the database program using an equation based on the law of spherical cosines (appendix 7). This facilitates comparisons to nearby samples in the database. However, nearby samples may represent one or more different beds so the user needs to confirm stratigraphic equivalency.

The COALQUAL web interface consists of four horizontal tabs (fig. 1), the Home tab, the Browse Coal Data tab, the Search and Download Data tab, and the Browse Miscellaneous Data tab.

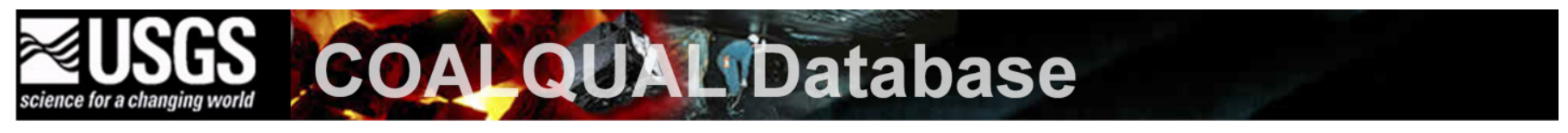

\begin{tabular}{|c|c|c|c|}
\hline Home & Browse Coal Data & Search and Download Data & Browse Miscellaneous Data \\
\hline
\end{tabular}

Figure 1. A screen image of the COALQUAL Version 3.0 web interface banner and tabs.

\section{Home Tab}

The Home tab (fig. 2) contains contact information and a link to generate a report in comma separated values (CSV) format, which shows the total number of samples that currently exist in the COALQUAL database. The index of samples is grouped by State, county, and sample type.

\section{Browse Coal Data Tab}

The Browse Coal Data tab provides a quick way to search data using the following search criteria related to samples: apparent rank, State, county, and coal province. When a State is selected from the State pull down menu, the County pull down menu is populated with the county names that belong to the selected State. When the Show button is clicked after selecting one or more of the search values, search results will be returned in table format in the left area of the tab as shown below in figure 3 .

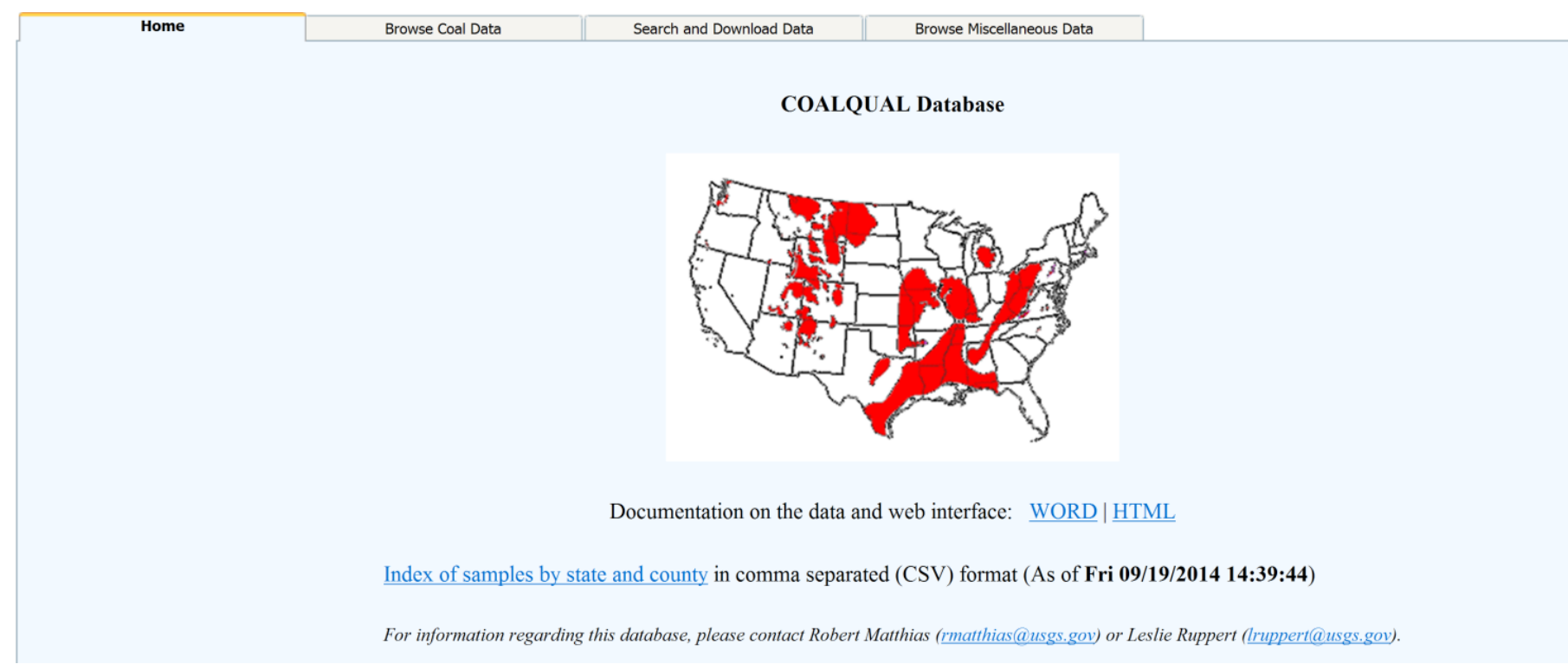

Figure 2. A screen image of the COALQUAL Version 3.0 Home tab. 
To see additional information on each sample, the user can select a sample record by clicking on a row in the table to see other samples related to the selected sample. Other samples located at the same location (as the selected sample) will be returned in a table on the top right area of the Browse Coal Data tab (fig. 3). Note that samples associated directly with the selected sample are highlighted in green. Samples located within one mile radius (or that originated from the same mine or power plant) as the selected sample will be returned in a table in the bottom right area of the tab (fig. 4).

Click on the plus (+) icon in front of each sample record to see subsamples related to the selected sample (fig. 5). For example, subsamples can be bench samples composited together to make up an Upper Level sample (highlighted green in fig. 5). The plus icon appears in front of each sample row only if a sample has related subsamples. When the selected row is expanded, the plus icon is replaced by a minus (-) icon to indicate that the expanded row can be collapsed to hide the related subsamples (highlighted in light blue) as shown in figure 5.

Double click on a sample record in any table within the Browse Coal Data tab to see the selected sample's information in detail. A new browser window will open to show the information related to the selected sample (fig. 6).

\section{Search and Download Data Tab}

The Search and Download Data tab (fig. 7) is equipped with extensive search criteria to provide users the ability to select advanced filtering options giving users the power to find samples that match very specific criteria. Initially, the Search and Download Data tab is preset with several default options. The pull down menu for the type of report (fig. 8) is set to Sample Descriptions which shows mainly the geologic location of samples. Note that the Oxides report shows the analysis values related to major elements.

The Report Format pull down menu (fig. 9) is set to the Display report in browser option to display search results in table format within a new browser window. The other two options can be used to download results in CSV format. The last download option in the menu produces two CSV files (fig. 10) compressed into a single ZIP folder:

a. The first CSV file contains only Upper Level samples.

b. The other file shows subsamples that make up the Upper Level samples shown in the first file mentioned in the previous step a. The first column called "Related To" contains the sample numbers of the Upper Level samples to show the relationship between subsamples and Upper Level samples. Note that combining both the Upper Level sample data and the subsample data requires extreme care to obtain accurate statistical results. Subsample data may represent multiple duplicate samples and partial-bed samples.

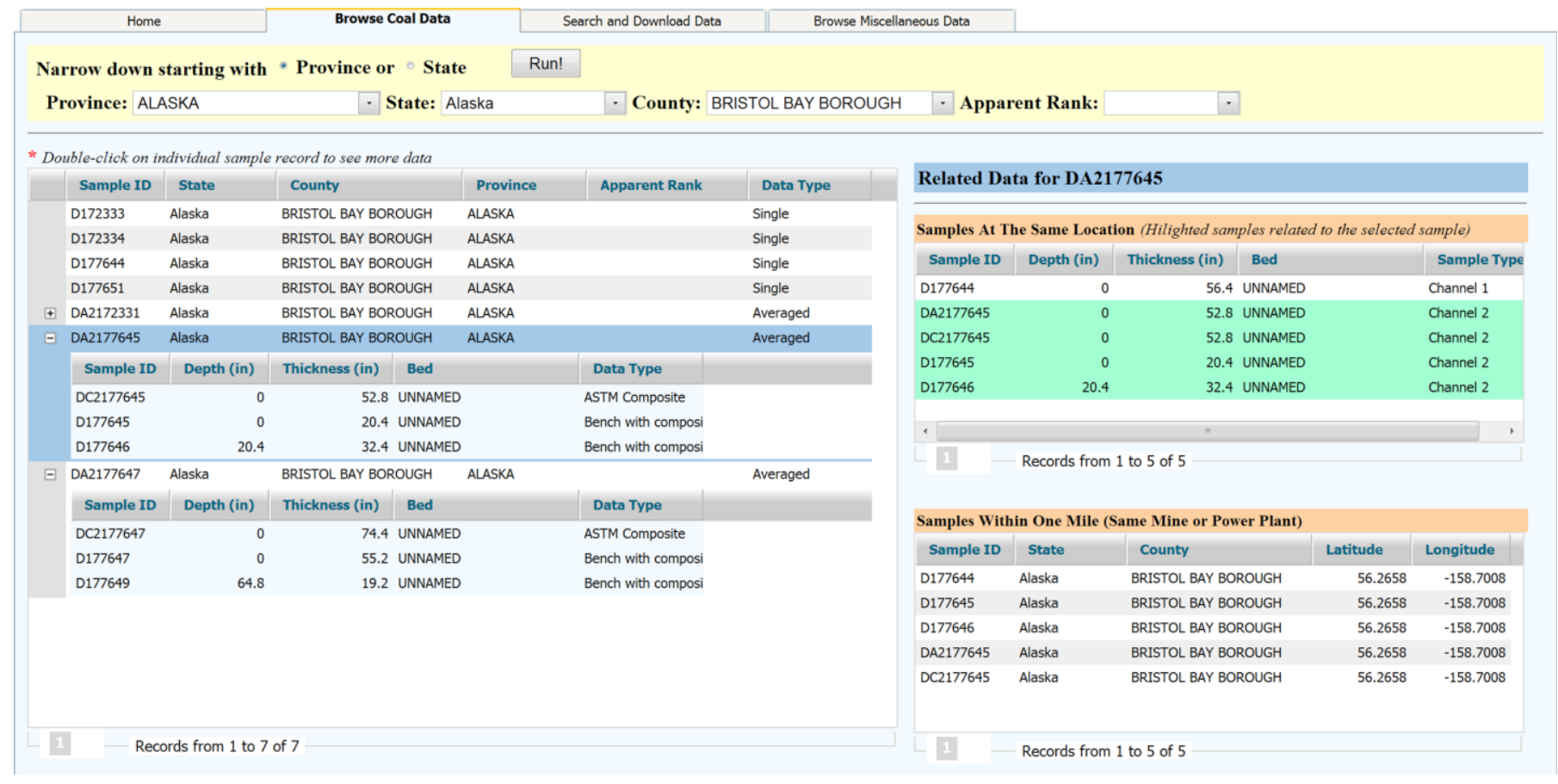

Figure 3. An example of the COALOUAL Version 3.0 Browse Coal Data tab and search screen. 
Figure 4. An example of coal samples that are from either the same location, or located at the same mine or power plant (within one mile distance).
Samples At The Same Location (Hilighted samples related to the selected sample)

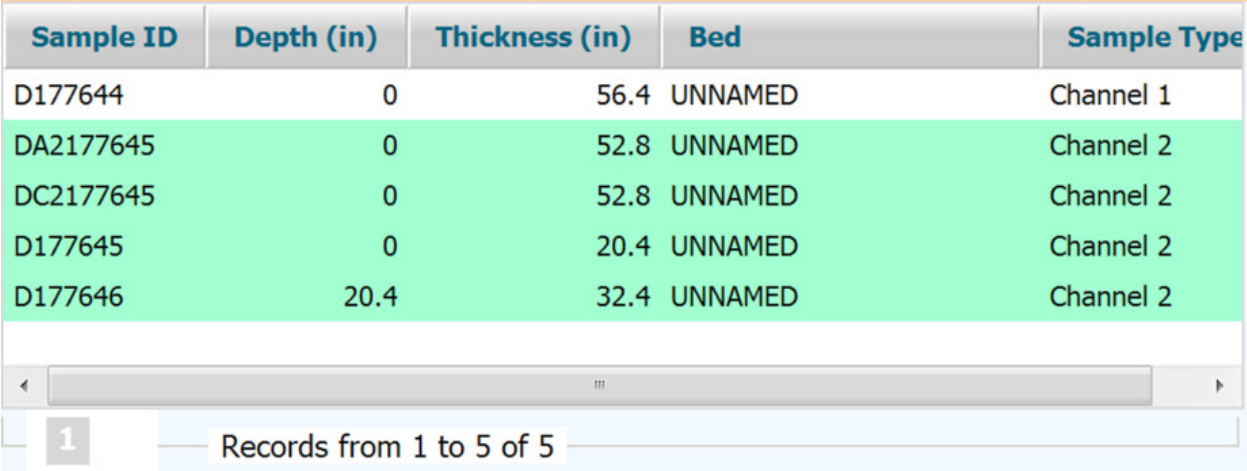

\begin{tabular}{|l|l|l|r|r|}
\hline \multicolumn{5}{|l|}{ Samples Within One Mile (Same Mine or Power Plant) } \\
\hline \multicolumn{1}{|c|}{ Sample ID } & State & County & \multicolumn{1}{l|}{ Latitude } & \multicolumn{1}{c|}{ Longitude } \\
\hline D177644 & Alaska & BRISTOL BAY BOROUGH & 56.2658 & -158.7008 \\
\hline D177645 & Alaska & BRISTOL BAY BOROUGH & 56.2658 & -158.7008 \\
\hline D177646 & Alaska & BRISTOL BAY BOROUGH & 56.2658 & -158.7008 \\
\hline DA2177645 & Alaska & BRISTOL BAY BOROUGH & 56.2658 & -158.7008 \\
\hline DC2177645 & Alaska & BRISTOL BAY BOROUGH & 56.2658 & -158.7008 \\
& & & & \\
\hline & & & \\
\hline
\end{tabular}

Figure 5. An example of the relationship of coal subsamples to an Upper Level sample.

\begin{tabular}{|c|c|c|c|c|c|c|c|}
\hline & Sample ID & State & \multicolumn{2}{|l|}{ County } & Province & Apparent Rank & Data Type \\
\hline & D172333 & Alaska & \multicolumn{2}{|c|}{ BRISTOL BAY BOROUGH } & \multicolumn{2}{|l|}{ ALASKA } & Single \\
\hline & D172334 & Alaska & \multicolumn{2}{|c|}{ BRISTOL BAY BOROUGH } & \multicolumn{2}{|l|}{ ALASKA } & Single \\
\hline & D177644 & Alaska & \multicolumn{2}{|c|}{ BRISTOL BAY BOROUGH } & \multicolumn{2}{|l|}{ ALASKA } & Single \\
\hline & D177651 & Alaska & \multicolumn{2}{|c|}{ BRISTOL BAY BOROUGH } & \multicolumn{2}{|l|}{ ALASKA } & Single \\
\hline+ & DA2172331 & Alaska & \multicolumn{2}{|c|}{ BRISTOL BAY BOROUGH } & \multicolumn{2}{|l|}{ ALASKA } & Averaged \\
\hline \multirow[t]{5}{*}{$\Xi$} & DA2177645 & Alaska & \multicolumn{2}{|c|}{ BRISTOL BAY BOROUGH } & \multicolumn{2}{|l|}{ ALASKA } & Averaged \\
\hline & Sample ID & Depth (in) & Thickness (in) & Bed & & Data Type & \\
\hline & DC2177645 & 0 & \multicolumn{3}{|c|}{52.8 UNNAMED } & ASTM Composite & \\
\hline & D177645 & 0 & \multicolumn{3}{|c|}{20.4 UNNAMED } & \multicolumn{2}{|l|}{ Bench with composi } \\
\hline & D177646 & 20.4 & \multicolumn{3}{|c|}{32.4 UNNAMED } & Bench with composi & \\
\hline \multirow[t]{5}{*}{$\square$} & DA2177647 & Alaska & \multicolumn{2}{|c|}{ BRISTOL BAY BOROUGH } & \multirow[t]{2}{*}{ ALASKA } & & Averaged \\
\hline & Sample ID & Depth (in) & Thickness (in) & Bed & & Data Type & \\
\hline & DC2177647 & 0 & \multicolumn{3}{|c|}{74.4 UNNAMED } & ASTM Composite & \\
\hline & D177647 & 0 & \multicolumn{3}{|c|}{55.2 UNNAMED } & Bench with composi & \\
\hline & D177649 & 64.8 & \multicolumn{3}{|c|}{19.2 UNNAMED } & Bench with composi & \\
\hline
\end{tabular}




\section{モUSGS COALQULD Database}

Sample Detail for W212981

\begin{tabular}{|c|c|c|c|c|c|c|c|}
\hline & & & & & View Definit & ions of Qualifier: & dParameter \\
\hline & mple Description & Proximate & Ultimate & Oxide & & Trace & ment \\
\hline Sample ID & W212981 & Sample ID & W212981 & Sample ID & W212981 & Sample ID & W212981 \\
\hline State & Alabama & Moisture & & Remnant Moisture & 4 & GS Ash Dry & 25.4 \\
\hline County & TUSCALOOSA & Moisture Q & $\underline{B}$ & Remnant Moisture Q & $\underline{\mathrm{e}}$ & GS Ash Dry Q & \\
\hline Latitude & 33.5272 & Volatile Matter & & GSAsh & 24.4 & $\mathrm{Si}$ & 44500 \\
\hline Longitude & -87.5100 & Volatile Matter Q & $\underline{B}$ & GSAsh Q & & $\mathrm{SiQ}$ & \\
\hline Province & EASTERN & Fixed Carbon & & $\mathrm{SiO}_{2}$ & 37.5 & $\mathrm{Al}$ & 33300 \\
\hline Region & SOUTHERN APPALACHIAN & Fixed Carbon Q & & $\mathrm{SiO}_{2} \mathrm{Q}$ & $\underline{\mathrm{o}}$ & $\mathrm{AlQ}$ & \\
\hline Field & WARRIOR & Standard Ash & & $\mathrm{Al}_{2} \mathrm{O}_{3}$ & 24.8 & $\mathrm{Ca}$ & 11400 \\
\hline District & & Standard Ash Q & $\underline{B}$ & $\mathrm{Al}_{2} \mathrm{O}_{3} \mathrm{Q}$ & $\underline{\mathrm{o}}$ & $\mathrm{CaQ}$ & \\
\hline Formation & POTTSVILLE & Proximate Validation & Incomplete Data & $\mathrm{CaO}$ & 6.27 & $\mathrm{Mg}$ & 1190 \\
\hline Group & PRATT & Hydrogen & & $\mathrm{CaOQ}$ & $\underline{\mathrm{o}}$ & $\mathrm{Mg} Q$ & \\
\hline Bed & AMERICAN & Hydrogen Q & $\underline{B}$ & $\mathrm{MgO}$ & 0.78 & $\mathrm{Na}$ & 763 \\
\hline Member & & Carbon & & $\mathrm{MgOQ}$ & $\underline{\mathrm{o}}$ & $\mathrm{Na} Q$ & \\
\hline Coal Zone & & Carbon Q & $\underline{B}$ & $\mathrm{MnO}$ & 0.023 & $\mathrm{~K}$ & 2490 \\
\hline Depth (in) & 10621.2 & Nitrogen & & $\mathrm{MnOQ}$ & & K Q & \\
\hline Thickness (in) & 10 & Nitrogen Q & $\underline{B}$ & $\mathrm{Na}_{2} \mathrm{O}$ & 0.405 & $\mathrm{Fe}$ & 32800 \\
\hline System & Pennsylvanian & Oxygen & & $\mathrm{Na}_{2} \mathrm{OQ}$ & 0 & $\mathrm{Fe} \mathrm{Q}$ & \\
\hline Series/Epoch & Lower & Oxygen Q & $\underline{B}$ & $\mathrm{~K}_{2} \mathrm{O}$ & 1.18 & $\mathrm{Ti}$ & 1550 \\
\hline Literature & & Sulfur & & $\mathrm{K}_{2} \mathrm{OQ}$ & 0 & TiQ & \\
\hline Comments & & Sulfur Q & $\underline{B}$ & $\mathrm{Fe}_{2} \mathrm{O}_{3}$ & 18.4 & s & 1.53 \\
\hline Map & BERRY SE (7.5') & Ultimate Validation & Incomplete Data & $\mathrm{Fe}_{2} \mathrm{O}_{3} \mathrm{Q}$ & o & S Q & $\underline{s, d}$ \\
\hline Collector & ALGS-WARD R & Btu & & $\mathrm{TiO}_{2}$ & 1.02 & $\mathrm{Ag}$ & 0.104 \\
\hline Mine/Power Plant & NO DATA ENTERED & Btu Q & $\underline{B}$ & $\mathrm{TiO}_{2} \mathrm{Q}$ & $\begin{array}{l}1.02 \\
0\end{array}$ & $\mathrm{Ag} \mathrm{Q}$ & \\
\hline Drill Core No & 266166-KII-40.H & Sulfate Sulfur & & $\mathrm{P}_{2} \mathrm{O}_{5}$ & $\underline{\underline{y}}$ & As & 111 \\
\hline Point Id & 266166-KII-40.H & Sulfate Sulfur Q & $\underline{B}$ & $\begin{array}{l}\mathrm{P}_{2} \mathrm{O}_{5} \\
\mathrm{P}, \mathrm{O}_{5} \mathrm{O}\end{array}$ & & As $Q$ & \\
\hline Submit Date & $03 / 30 / 1981$ & Pyritic Sulfur & & $\begin{array}{l}\mathrm{P}_{2} \mathrm{O}_{5} \mathrm{Q} \\
\mathrm{SO}_{3}\end{array}$ & 3.68 & $\mathrm{Au}$ & 2.6 \\
\hline Sample Description & BITUMINOUS COAL & Pyritic Sulfur Q & $\underline{B}$ & $\begin{array}{ll}\mathrm{SO}_{3} \\
\mathrm{SO}_{3} \mathrm{O}\end{array}$ & 3.08 & $\mathrm{Au} \mathrm{Q}$ & $\underline{\mathrm{L}}$ \\
\hline Estimated Rank & BITUMINOUS & Organic Sulfur & & $\mathrm{SO}_{3} \mathrm{Q}$ & $\underline{s}$ & B & 55.9 \\
\hline Apparent Rank & & Organic Sulfur Q & $\underline{B}$ & LOI & & BQ & \\
\hline Analytical Labs & USBM and USGS & Ash Deformation & & & & $\mathrm{Ba}$ & 661 \\
\hline Sample Type & Drill Core & Ash Deformation Q & $\underline{B}$ & & & $\mathrm{Ba} \mathrm{Q}$ & \\
\hline Analysis Type & As Received & Ash Softening & & & & $\mathrm{Be}$ & 3.05 \\
\hline Values Represent & Single sample & Ash Softening Q & $\underline{B}$ & & & $\mathrm{Be} \mathrm{Q}$ & \\
\hline Township & & Ash Fluid & & & & $\mathrm{Bi}$ & 2.6 \\
\hline Range & & Ash Fluid Q & $\underline{B}$ & & & $\mathrm{BiQ}$ & $\underline{\mathrm{L}}$ \\
\hline Section & & Free Swelling & & & & $\mathrm{Br}$ & 5.42 \\
\hline Quarters & & Free Swelling Q & $\underline{B}$ & & & $\mathrm{BrQ}$ & \\
\hline Tract & & HGI & & & & $\mathrm{Cd}$ & 0.153 \\
\hline Location Detail & & HGI Q & & & & $\mathrm{Cd} \mathrm{Q}$ & \\
\hline
\end{tabular}

Figure 6. An example of the COALOUAL Version 3.0 coal Sample Detail page.

\begin{tabular}{|l||c|c|}
\hline \multicolumn{1}{|c|}{ Home } & Browse Coal Data & Bearch and Download Data \\
\hline Report: Sample Descriptions & - Report Format: & Display report in browser \\
\hline Search Coal Samples & $\circ$ Search Miscellaneous Samples \\
Qualifier L (less than) and G (greater than) : Exclude values with L and G qualifiers & Search Criteria Defined \\
\hline
\end{tabular}

Figure 7. A screen image of the COALQUAL Version 3.0 Search and Download Data tab.

Sample Descriptions Sample Descriptions

\section{Oxides}

Proximate \& Ultimate Analysis

Trace Elements

Figure 8. The pull down menu for report types on the Search and Download Data tab.
Display report in browser

\section{Display report in browser}

Download report in comma separated (CSV) format Download report in comma separated (CSV) format with related data

Figure 9. The Report Format pull down menu on the Search and Download Data tab. 
Figure 10. Examples of two CSV files showing Upper Level samples and related subsamples.

\begin{tabular}{|c|c|c|c|c|c|c|c|}
\hline \multicolumn{8}{|c|}{ CQ2012321104720.CSV [Read-Only] } \\
\hline$\triangle$ & A & B & \multicolumn{2}{|l|}{ C } & D & $\mathrm{E}$ & $\mathrm{F}$ \\
\hline 1 & Sample ID & State & \multicolumn{2}{|l|}{ County } & Latitude L & Longitude & Province \\
\hline 2 & W233974 & Alabama & \multicolumn{2}{|c|}{ TUSCALOOSA } & 37.3278 & -87.2092 & EASTERN \\
\hline 3 & W233978 & Alabama & \multicolumn{2}{|c|}{ TUSCALOOSA } & 33.2703 & -87.3739 & EASTERN \\
\hline 4 & WA2204687 & Alabama & \multicolumn{2}{|c|}{ TUSCALOOSA } & 33.4856 & -87.5414 & EASTERN \\
\hline 5 & WA2211667 & Alabama & \multicolumn{2}{|c|}{ TUSCALOOSA } & 33.5717 & -87.47 & EASTERN \\
\hline 6 & WA2213006 & Alabama & \multicolumn{2}{|c|}{ TUSCALOOSA } & 33.59 & -87.4911 & EASTERN \\
\hline 7 & & & & & & & \\
\hline \multicolumn{7}{|c|}{ 빨] CQ2012321104720S.CSV [Read-Only] } & 口 回 \\
\hline$\Delta$ & $\mathrm{A}$ & $B$ & C & & D & $E$ & $\mathrm{~F}$ \\
\hline 1 & Related To & Sample ID & State & Cou & unty & Latitude & Longitude \\
\hline 2 & WA2204687 & W204686 & Alabama & TUS & SCALOOSA & 33.4856 & -87.541 \\
\hline 3 & WA2204687 & W204687 & Alabama & TUS & SCALOOSA & 33.4856 & -87.541 \\
\hline 4 & WA2211667 & W211667 & Alabama & TUS & SCALOOSA & 33.5717 & -87.4 \\
\hline 5 & WA2211667 & W211669 & Alabama & TUS & SCALOOSA & 33.5717 & -87.4 \\
\hline 6 & WA2213006 & W213006 & Alabama & TUS & SCALOOSA & 33.59 & -87.491 \\
\hline 7 & WA2213006 & W213007 & Alabama & TUS & SCALOOSA & 33.59 & -87.491 \\
\hline 8 & & & & & & & \\
\hline
\end{tabular}

Also note that the Report Format menu works in conjunction with the Report menu. For example, if the Proximate \& Ultimate Analysis report is selected, search results will only show attributes associated with the proximate and ultimate analysis.

The search results window displays the search criteria defined by the user (and a table) as shown in figure 11. Radio buttons allow the user to search either coal samples or miscellaneous samples, but not both (fig. 12). By default, the Search Coal Samples option is selected to limit the search to coal samples.

The pull down menu (fig. 13) for data qualifiers (L for less than and G for greater than) is set to the Exclude values with $\mathbf{L}$ and $\mathbf{G}$ qualifiers option to filter out samples that have analysis values with $\mathrm{L}$ or $\mathrm{G}$ qualifiers. This pull down menu is only applicable when the user selects one or more attributes related to analysis values. The other options can be used to limit the search on samples that have analysis that include (1) values with L qualifier, (2) values with G qualifier, or (3) values with $\mathrm{L}$ and G qualifiers.

The Search Criteria Defined checkbox (fig. 14) will show a check mark when one or more search options are defined by the user. At any time, the current active search criteria can be displayed by clicking Show Criteria. When the Show Criteria button is clicked after defining a search criteria, a new window will open showing selected criteria for an additional search (fig. 15). Search criteria are divided into four categories (fig. 16), which are closely associated with the four types of reports and represented by the following four tabs: Sample Description, Proximate/Ultimate, Oxides, and Trace element.

\section{Sample Description Tab}

The Sample Description tab (fig. 17) consists of search criteria mainly associated with the geologic location of samples.

\section{Proximate/Ultimate Tab}

The Proximate/Ultimate tab (fig. 18) allows the user to search samples by defining ranges of values associated with proximate and (or) ultimate analysis. 


\section{ZUSGS COALQULD Database}

Proximate and ultimate analysis, heat of combustion, forms of sulfur, free-swelling index, and ash-fusion temperature determination for selected coal samples.

[All analyses in percent except free-swelling index, ash fusion temperatures, Btu/lb, and Btu related analyses. For each sample number, the analyses are reported as determined. Analyses are by a commercial testing laboratory following ASTM standards.]

Your search criteria were: (Btu between 12000 and 15000)) and (Exclude Btu with L or G qualifiers) and (STDASH between 10 and 15 )) and (Exclude STDASH with L or G qualifiers) View Definitions of Qualifiers and Parameters

\begin{tabular}{|c|c|c|c|c|c|c|c|c|c|c|c|c|c|c|c|c|c|}
\hline & \multirow[b]{2}{*}{ Sample ID } & \multicolumn{9}{|c|}{ Proximate Analysis } & \multicolumn{7}{|c|}{ Ultimate Analysis } \\
\hline & & Moisture & $\begin{array}{l}\text { Moisture } \\
\text { Q }\end{array}$ & $\begin{array}{l}\text { Volatile } \\
\text { Matter }\end{array}$ & $\begin{array}{l}\text { Volatile } \\
\text { Matter Q }\end{array}$ & $\begin{array}{l}\text { Fixed } \\
\text { Carbon }\end{array}$ & $\begin{array}{l}\text { Fixed } \\
\text { Carbon Q }\end{array}$ & $\begin{array}{l}\text { Standard } \\
\text { Ash }\end{array}$ & $\begin{array}{l}\text { Standard } \\
\text { Ash Q }\end{array}$ & $\begin{array}{l}\text { Proximate } \\
\text { Validation }\end{array}$ & Hydrogen & $\begin{array}{l}\text { Hydrogen } \\
\mathbf{Q}\end{array}$ & Carbon & $\begin{array}{l}\text { Carbon } \\
\mathbf{Q}\end{array}$ & Nitrogen & $\begin{array}{l}\text { Nitrogen } \\
\mathbf{Q}\end{array}$ & Oxygen \\
\hline \multirow[t]{4}{*}{$\boxminus$} & WA2190637 & 2.94 & a & 29.62 & a & 56.97 & & 10.47 & a & Acceptable & 4.61 & a & 74.4 & a & 1.5 & a & 3.13 \\
\hline & Sample ID & Depth (in) & Thickness (i & (in) & Bed & & Data Type & & & & & & & & & & \\
\hline & W190637 & & 0 & $26.75 \mathrm{E}$ & AGLE & & Bench & & & & & & & & & & \\
\hline & W190638 & 26. & & $15.5 \mathrm{E}$ & AGLE & & Bench & & & & & & & & & & \\
\hline+ & WA2191112 & 2.78 & a & 26.99 & a & 57.42 & & 12.81 & a & Acceptable & 4.56 & a & 73.31 & a & 1.25 & a & 3.6 \\
\hline$\oplus$ & WA2191122 & 2.79 & a & 30.66 & a & 55.54 & & 11.02 & a & Acceptable & 4.74 & a & 73.76 & a & 1.05 & a & 4.15 \\
\hline 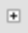 & WA2191324 & 2.08 & a & 32.75 & a & 52.48 & & 12.7 & a & Acceptable & 4.59 & a & 72.58 & a & 1.27 & a & 3.83 \\
\hline$\oplus$ & WA2191327 & 2.52 & a & 36.14 & a & 49.57 & & 11.77 & a & Acceptable & 4.75 & a & 71.98 & a & 1.42 & a & 5.57 \\
\hline
\end{tabular}

Figure 11. An example of search results filtered by search criteria.

\section{- Search Coal Samples • Search Miscellaneous Samples}

Figure 12. A screen image of the radio buttons for options to search coal or miscellaneous samples.

\section{Exclude values with $L$ and $G$ qualifiers . Exclude values with $L$ and $G$ qualifiers Values with $L$ qualifier Values with $G$ qualifier Include values with $L$ and $G$ qualifiers}

Figure 13. A screen image of the pull down menu for the $L$ and $G$ data qualifiers.

\section{Search Criteria Defined}

\section{Show Criteria}

Figure 14. A screen image of the check box and button for search criteria.

\section{Proximate/Ultimate}

(Btu between 12000 and 15000)

Exclude Btu with $\mathrm{L}$ or $\mathrm{G}$ qualifiers

(STDASH between 10 and 15)

Exclude STDASH with L or G qualifiers

Figure 15. An example of additional search criteria. 
Sample Description

Proximate/Ultimate

\section{Oxides}

Trace element

Figure 16. A screen image of the four search categories.

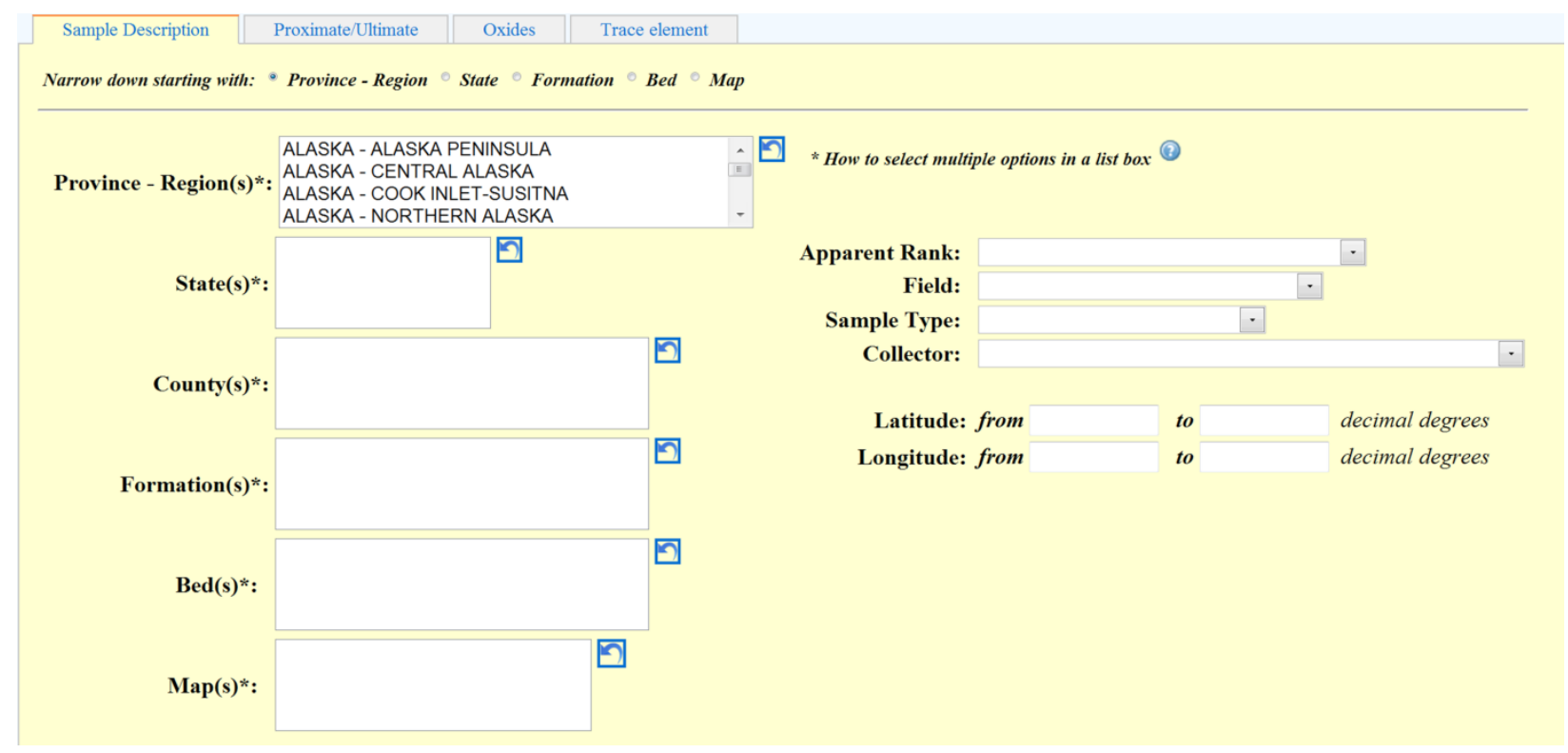

Figure 17. The search criteria in the Sample Description tab.

\begin{tabular}{|c|c|c|c|}
\hline Sample Description & \multicolumn{2}{|c|}{ Oxides } & Trace element \\
\hline \multicolumn{2}{|l|}{ Category } & from & to \\
\hline Btu (Btu/lb) & $\cdot$ & 12000 & 15000 \\
\hline Standard Ash (percent) & - & 10 & 15 \\
\hline -- None selected -- & $\cdot$ & & \\
\hline -- None selected -- & - & & \\
\hline -- None selected -- & $\cdot$ & & \\
\hline -- None selected -- & $\cdot$ & & \\
\hline -- None selected -- & - & & \\
\hline -- None selected -- & $\cdot$ & & \\
\hline -- None selected -- & $\cdot$ & & \\
\hline -- None selected -- & $\cdot$ & & \\
\hline
\end{tabular}

Figure 18. The search criteria in the Proximate/Ultimate tab. 


\section{Oxides Tab}

The Oxides tab (fig. 19) allows the user to select samples by adding ranges of values associated with the analysis of oxides.

\section{Trace Elements Tab}

The Trace element tab (fig. 20) lets the user query the database with ranges of values related to the analysis of major, minor, and trace elements.

\section{Browse Miscellaneous Data Tab}

The Browse Miscellaneous Data tab is implemented to show non-coal, miscellaneous samples based on four search criteria related to samples, as follows: State and County (location), Sample Type, and Rejected (reason for rejection as an unacceptable coal sample). Just like the State and County pull down menus in the Browse Coal Data tab, when a State is selected from the State pull down menu, the County pull down menu gets populated with the county names that belong to the selected State. After selecting values from the search criteria, clicking on the Show button will return miscellaneous samples in a table as displayed in figure 21 .

To see additional information on each sample, double click on a sample record. A new internet browser window will open with sample data related to the selected sample (fig. 22).

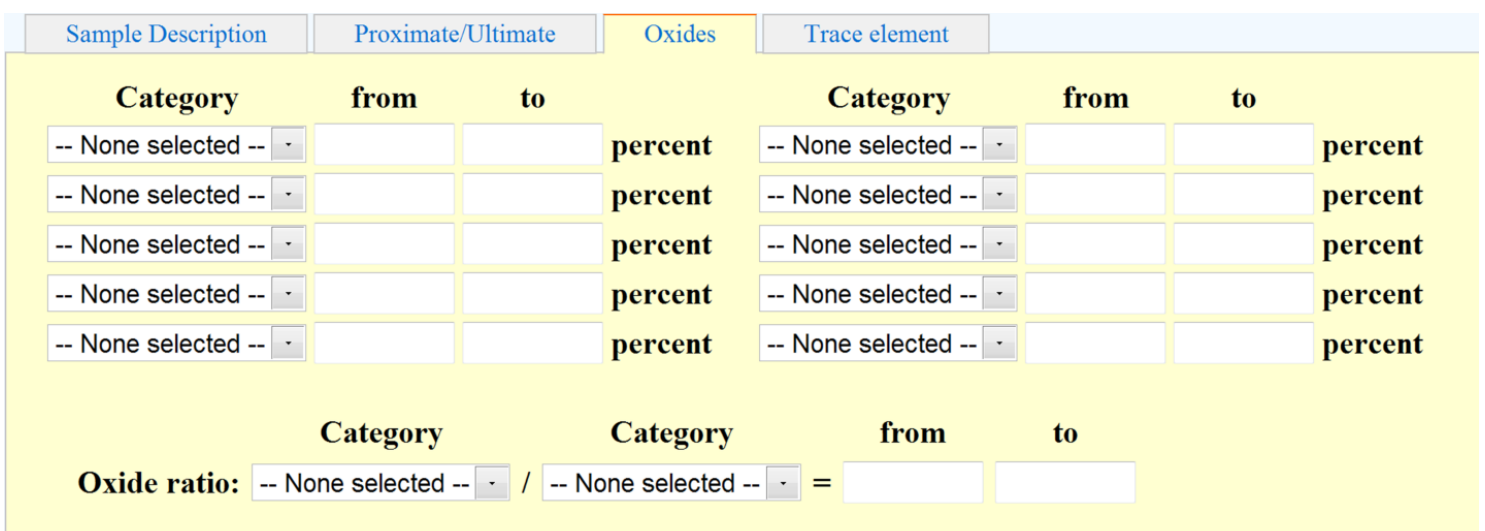

Figure 19. The search criteria in the 0xides tab.

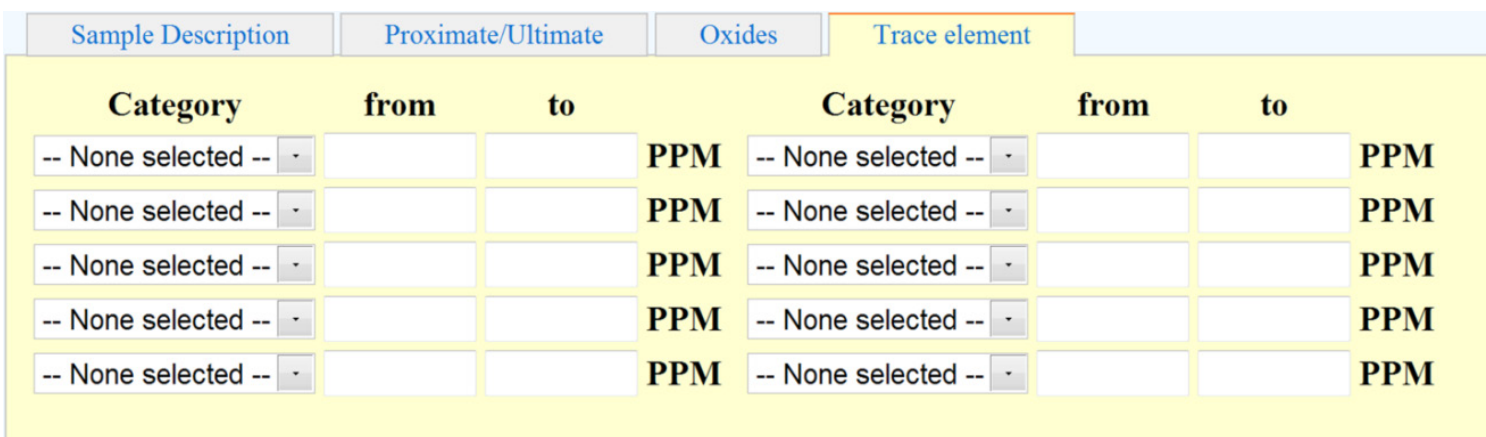

Figure 20. The search criteria in the Trace element tab. [PPM, parts per million] 


\begin{tabular}{|c|c|c|c|c|c|c|c|c|c|}
\hline \multicolumn{2}{|r|}{ Home } & \multicolumn{2}{|c|}{ Browse Coal Data } & \multicolumn{2}{|c|}{ Search and Download Data } & Browse Miscellaneous Data & \multirow[b]{2}{*}{ Rejected: } & \multirow[b]{2}{*}{$\cdot$} & \multirow[b]{2}{*}{ Show } \\
\hline State: & Wyoming & - County: & CAMPBELL & $\cdot$ & Sample Type: & $\cdot$ & & & \\
\hline \multicolumn{10}{|c|}{ * Double-click on individual Sample record to see more data } \\
\hline Sample ID & State & County & Province & Apparent Rank & Rejected & Sample Type & Values Represent & & \\
\hline D160981 & Wyoming & CAMPBELL & NORTHERN GREAT & & ROCK & Channel & Single sample & & \\
\hline D160986 & Wyoming & CAMPBELLL & NORTHERN GREAT & & RUN OF MINE & Run of Mine & Physical or mathemtical composite (by thickness) & & \\
\hline D160987 & Wyoming & CAMPBELL & NORTHERN GREAT & & TIPPLE & Tipple & Physical or mathemtical composite (by thickness) & & \\
\hline D160988 & Wyoming & CAMPBELL & NORTHERN GREAT & & TIPPLE & Tipple & Physical or mathemtical composite (by thickness) & & \\
\hline D161800 & Wyoming & CAMPBELL. & NORTHERN GREAT & & ROAD CUT BURNED & Naturally Burned Roa & High ash yield samples & & \\
\hline D161801 & Wyoming & CAMPBELL & NORTHERN GREAT & & ROAD CUT BURNED & Naturally Burned Roa & High ash yield samples & & \\
\hline D163196 & Wyoming & CAMPBELL & NORTHERN GREAT & & NATURALLY BURNED & Naturally Burned & High ash yield samples & & \\
\hline D163516 & Wyoming & CAMPBELL & NORTHERN GREAT & & WEATHERED & Channel & Bench & & \\
\hline D163517 & Wyoming & CAMPBELL & NORTHERN GREAT & & WEATHERED & Channel & Bench & & \\
\hline D163518 & Wyoming & CAMPBELL & NORTHERN GREAT & & WEATHERED & Channel & Bench & & \\
\hline D163519 & Wyoming & CAMPBELL & NORTHERN GREAT & & WEATHERED & Channel & Bench & & \\
\hline D163520 & Wyoming & CAMPBELLL & NORTHERN GREAT & & WEATHERED & Channel & Bench & & \\
\hline D163521 & Wyoming & CAMPBELL & NORTHERN GREAT & & WEATHERED & Channel & Bench & & \\
\hline D163522 & Wyoming & CAMPBELL & NORTHERN GREAT & & TIPPLE & Tipple & Physical or mathemtical composite (by thickness) & & \\
\hline D164323 & Wyoming & CAMPBELLL & NORTHERN GREAT & & DRILL ADDITIVE & Drill Fluid additive & Single sample & & \\
\hline D164324 & Wyoming & CAMPBELL & NORTHERN GREAT & & DRILL ADDITIVE & Drill Fluid additive & Single sample & & \\
\hline D164325 & Wyoming & CAMPBELL & NORTHERN GREAT & & DRILL ADDITIVE & Drill Fluid additive & Single sample & & \\
\hline D164326 & Wyoming & CAMPBELL & NORTHERN GREAT & & DRILL ADDITIVE & Drill Fluid additive & Single sample & & \\
\hline D164327 & Wyoming & CAMPBELL & NORTHERN GREAT & & DRILL ADDITIVE & Drill Fluid additive & Single sample & & \\
\hline D164604 & Wyoming & CAMPBELL & NORTHERN GREAT & & LOW RECOVERY & Drill Core & Core with low recovery $(<70 \%)$ & & \\
\hline
\end{tabular}

Figure 21. A screen image of the Browse Miscellaneous Data tab.

\section{ZUSGS COALQUALDatabase}

Sample Detail for D176537

\begin{tabular}{|c|c|c|c|c|c|c|c|}
\hline \multirow{2}{*}{\multicolumn{2}{|c|}{ Sample Description }} & & & \multicolumn{4}{|c|}{ View Definitions of Oualifiers and Parameters } \\
\hline & & \multicolumn{2}{|c|}{ Proximate \& Ultimate } & \multicolumn{2}{|c|}{ Oxide } & \multicolumn{2}{|c|}{ Trace Element } \\
\hline Sample ID & D176537 & Sample ID & D176537 & Sample ID & D176537 & Sample ID & D176537 \\
\hline State & Wyoming & Moisture & & Remnant Moisture & 0 & GS Ash Dry & 78.6 \\
\hline County & CAMPBELL & Moisture Q & & Remnant Moisture Q & e & GS Ash Dry Q & \\
\hline Latitude & 44.8117 & Volatile Matter & & GSAsh & 78.6 & $\mathrm{Si}$ & 110000 \\
\hline Longitude & -105.5908 & Volatile Matter Q & & GSAsh Q & & $\mathrm{SiQ}$ & d \\
\hline Province & NORTHERN GREAT PLAINS & Fixed Carbon & & $\mathrm{SiO}_{2}$ & 30 & $\mathrm{Al}$ & 37000 \\
\hline Region & POWDER RIVER & Fixed Carbon Q & & $\mathrm{SiO}_{2} \mathrm{Q}$ & & $\mathrm{AlQ}$ & d \\
\hline Field & LITTLE POWDER RIVER & Standard Ash & & $\mathrm{Al}_{2} \mathrm{O}_{3}$ & 8.9 & $\mathrm{Ca}$ & 26900 \\
\hline District & & Standard Ash Q & & $\mathrm{Al}_{2} \mathrm{O}_{3} \mathrm{O}$ & & $\mathrm{Ca} \mathrm{Q}$ & d \\
\hline Formation & FORT UNION & \begin{tabular}{|l|} 
Proximate Validation \\
\end{tabular} & & $\begin{array}{ll}\mathrm{H}_{2} \mathrm{O}_{3} \mathrm{~V} \\
\mathrm{CaO}\end{array}$ & 48 & $\mathrm{Mg}$ & 17000 \\
\hline Group & & Hydrogen & & \begin{tabular}{|l|l|l}
$\mathrm{CaO}$ \\
$\mathrm{CaOO}$
\end{tabular} & 4.8 & $\mathrm{Mg} \mathrm{Q}$ & d \\
\hline Bed & & Hydrogen Q & & $\mathrm{MaO}$ & 3.58 & $\mathrm{Na}$ & 990 \\
\hline Member & TONGUE RIVER & Carbon & & $\mathrm{MgO}$ & 5.58 & $\mathrm{Na} Q$ & d \\
\hline Coal Zone & TONGUE RIVER & Carbon Q & & \begin{tabular}{|l}
$\mathrm{MgO} \mathrm{Q}$ \\
$\mathrm{MnO}$
\end{tabular} & 0.206 & $\mathrm{~K}$ & 7190 \\
\hline Depth (in) & 1612.8 & \begin{tabular}{|l} 
Nitrogen \\
\end{tabular} & & \begin{tabular}{|l|}
$\mathrm{MnO}$ \\
$\mathrm{MnO} O$ \\
\end{tabular} & 0.206 & K Q & d \\
\hline Thickness (in) & 8.4 & Nitrogen Q & & $\mathrm{MnO} Q$ & & $\mathrm{Fe}$ & 264000 \\
\hline System & Tertiary & Oxygen & & $\mathrm{Na}_{2} \mathrm{O}$ & 0.17 & $\mathrm{Fe} Q$ & d \\
\hline Series/Epoch & Paleocene & Oxygen Q & & $\mathrm{Na}_{2} \mathrm{O} Q$ & & $\mathrm{Ti}$ & 1740 \\
\hline Literature & & Sulfur & & $\mathrm{K}_{2} \mathrm{O}$ & 1.1 & TiQ & d \\
\hline Comments & & Sulfur Q & & $\mathrm{K}_{2} \mathrm{O} \mathrm{Q}$ & & S & 0.1 \\
\hline Map & WHITE TAIL BUTTE $\left(7.5^{\prime}\right)$ & Ultimate Validation & & $\mathrm{Fe}_{2} \mathrm{O}_{3}$ & 48 & S Q & $\mathrm{L}$ \\
\hline Collector & USGS-HOBBS R G & Btu & & $\mathrm{Fe}_{2} \mathrm{O}_{3} \mathrm{Q}$ & & $\mathrm{Ag}$ & \\
\hline Mine/Power Plant & NO DATA ENTERED & Btu Q & & $\mathrm{TiO}_{2}$ & 0.37 & $\mathrm{Ag} \mathrm{Q}$ & \\
\hline Drill Core No & HOLE NO RM-3 & \begin{tabular}{|l|} 
Sulfate Sulfur \\
\end{tabular} & & $\mathrm{TiO}_{2} \mathrm{Q}$ & & As & 8 \\
\hline Point Id & RM-3-RS-6 & Sulfate Sulfur Q & & $\mathrm{P}_{2} \mathrm{O}_{5}$ & & As Q & \\
\hline Submit Date & $07 / 21 / 1975$ & Pyritic Sulfur & & $\mathrm{P}_{2} \mathrm{O}_{5} \mathrm{Q}$ & & $\mathrm{Au}$ & \\
\hline Sample Description & ROCK & Pyritic Sulfur Q & & & & $\mathrm{AuQ}$ & \\
\hline Apparent Rank & & \begin{tabular}{|l|} 
Organic Sulfur \\
\end{tabular} & & $\mathrm{SO}_{3}$ & 0.1 & $\mathrm{~B}$ & 40 \\
\hline Analytical Labs & U.S. Geological Survey (USGS) & Organic Sulfur Q & & $\mathrm{SO}_{3} \mathrm{Q}$ & s.L & $\mathrm{B} Q$ & $\underline{L}$ \\
\hline Sample Type & Rock Core sample & Ash Deformation & & LOI & & $\mathrm{Ba}$ & 550 \\
\hline
\end{tabular}

Figure 22. A detailed example of miscellaneous data. 


\section{Error Estimation}

Several errors associated with using these data were also estimated. Rules associated with error estimation, when combining bench samples to create a Upper Level sample, are given in appendix 5. Errors associated with remnant moisture effect on all elemental data, as well as the determination of whether a sample is a rock or coal, are discussed in appendix 4.

In some cases, only the type of error is identified. For example, qualifiers are used when data are incomplete or approximated using various procedures (appendix 3). This resulted in two levels of qualifiers. Those with a capital letter provided some additional information, but often not enough to allow the parameter to be used for statistical purposes. Small letter qualifiers were used for alternate procedures, which generally had smaller errors associated with them, and did not require censor from statistical procedures. In some instances, these qualifiers identified cases where data were not analyzed in complete compliance with ASTM standards, but errors were determined to be insignificant in determining the final value.

\section{Data Validation}

The degree of consistency and reliability of analytical results for any database is always a concern. Version 3.0 of COALQUAL represents the first attempt to develop verified data by crosschecking certain analytical parameters. The verification process is based on the fact that data interrelationships are often axiomatic in coal analyses. As the analytical result for one parameter changes, the results for related parameters also change. The pair of test results may vary inversely or be in direct proportion to each other. Correlations based on these data interrelationships can be used to estimate missing data (Hoeft and others, 1983). Data verification was performed on two suites of analyses, the ultimate and proximate data.

\section{Data Validation Methodology}

One of P.L. Dulong's contributions to coal chemistry is the idea that the heat of combustion of coal can be estimated by summing the weighted heats of combustion of their constituent elements (Francis and Lloyd, 1983). While it is widely circulated, P.L. Dulong never formally published his equation. By applying this principle to coal, predictive formulas have been developed to estimate higher heating values (HHV). A good predictive correlation is useful as an additional quality control tool, and it provides an independent measure of consistency.

The data validation in COALQUAL Version 3.0 is based on a proprietary algorithm developed by Quality Associates International Ltd., which is a refinement of the Dulong approach. This algorithm uses carbon, hydrogen, sulfur, and nitrogen values from the ultimate analyses to derive a predicted HHV. For each sample, the predicted HHV value is then compared to the measured HHV. The following relative rating scale was developed to rate the degree of agreement between the predicted and measured HHV results:

- EXCELLENT: HHV results are within 1 percent relative.

- GOOD: HHV results are within 2 percent relative.

- ACCEPTABLE: HHV results are within 3 percent relative.

- INVESTIGATE: HHV results exceed 3 percent relative.

Those samples that exceed 3 percent should be further investigated to determine a potential cause. For the Upper Level coal analyses in COALQUAL Version 3.0, 961 samples had either insufficient data or no HHV data.

The verification of the proximate analyses is based on the data interrelationships between parameters from the proximate and ultimate analyses. The proximate analysis values of residual moisture (moisture in the analytical sample), ash yield, and volatile matter are used to calculate fixed carbon by difference. Subtracting the fixed carbon from carbon from the ultimate analysis yields volatile carbon (dividing this result by 12 yields the number of moles of volatile carbon). Dividing the moles of volatile carbon by the number of moles of hydrogen from the ultimate analysis yields the number of moles of volatile coal, which should be between 0.0 and 0.5 moles. This validation serves to confirm the reliability of the proximate results, as well as the reliability of the carbon and hydrogen from the ultimate analyses. Results that fall in the 0.0 to 0.5 range are designated as ACCEPTABLE. Results that do not fall in the 0.0 to 0.5 range are designated as INVESTIGATE. An "incomplete" data designation indicates that a sample is missing critical test results required to carry out the validation process. 


\section{Validation Results}

COALQUAL Version 2.0 (Bragg and others, 1997) was submitted to Quality Associates International Ltd. for validation. A validation algorithm was applied to the ultimate analysis values and HHV. Although about 98 percent of all ultimate and HHV results passed at least the ACCEPTABLE quality level (as specified by the validation algorithm) the validation revealed the following problems:

1. The hydrogen and oxygen data recorded in the 1997 version included the hydrogen and oxygen associated with the moisture in the coal. Conventional coal industry practice does not include the hydrogen and oxygen in water when reporting these parameters in coal. The USGS corrected the hydrogen and oxygen numbers to exclude the hydrogen and oxygen from water in COALQUAL Version 3.0.

2. Approximately 3 percent of the samples failed to meet the ACCEPTABLE quality level set by the validation algorithm. The USGS attempted to verify whether any of these failures were a result of data entry errors, and determined that about 600 samples lacked all of the data required to carry out an ultimate and HHV validation. The USGS attempted to determine reasons for missing data.

During the investigation of these two validation problems it became evident that some composites consisted of combinations of bench samples from different lateral locations in the same bed, especially in the western United States, where unusually thick coal beds are encountered. If the results from both bench samples are ACCEPTABLE, then any properly calculated mathematical combination would also be ACCEPTABLE. However, such a combination would not be representative of the bed at a specific vertical sampling location. This revelation required a complete re-evaluation of all samples in the 1997 database to ensure bench samples had been properly composited. This resulted in the removal of a number of composite samples from the database, as well as recombination of properly selected benches to yield new composites. This process resulted in the Version 3.0 database, which contains 7,658 samples.

The edited COALQUAL Version 3.0 database was sent to Quality Associates International Ltd. for validation using the same algorithm (for ultimate and HHV) as used for the 1997 version, as well as the additional algorithm for validation of proximate data. The validation proceeded as a series of verification steps. It was confirmed for all 5,517 samples (that were also in the 1997 database) that the hydrogen and oxygen results did not originate from the water in coal in Version 3.0. Furthermore, the validation confirmed the ultimate and HHV ratings had not changed for these 5,517 coal samples. These two steps suggest there were no errors introduced in the correction of the hydrogen and oxygen data or in the transfer of entire datasets from the 1997 Version 2.0 database. We conclude the same level of scrutiny was employed when entering the 2,141 additional samples into the Version 3.0 database. This is confirmed in part by the validation of ultimate and HHV results of the COALQUAL Version 3.0 database, which resulted in 6,545 of the 6,683 complete datasets of ultimate and HHV results passing an ACCEPTABLE or better rating (97.94 percent of the complete sets passed), an improvement over the 1997 database Version 2.0 where 97.5 percent of the complete datasets passed. The summary of results for algorithm verification of the ultimate and $\mathrm{HHV}$ is shown in table 1.

Table 1. Validation summary for the degree of agreement between the predicted higher heating value (HHV) and the ultimate and measured HHV analyses. [Validated samples $(6,683)$ equals the total number of samples $(7,644)$ minus the number of incomplete samples (961), and refers to the number of complete datasets. Percent $(\%)$ is of 6,683 .]

\begin{tabular}{|c|c|c|}
\hline \multicolumn{3}{|c|}{ Ultimate and HHV Validation } \\
\hline \multirow{3}{*}{ Number of samples } & Total & 7,644 \\
\hline & Incomplete & 961 \\
\hline & Validated & 6,683 \\
\hline \multirow{4}{*}{ Rating scale } & EXCELLENT & $71.55 \%$ \\
\hline & GOOD & $21.44 \%$ \\
\hline & ACCEPTABLE & $4.95 \%$ \\
\hline & INVESTIGATE & $2.05 \%$ \\
\hline
\end{tabular}


There is a difference in the quality rating scales applied to ultimate and HHV validation versus that for proximate value verification. The ultimate and HHV algorithm is based on thermo-chemical calculations derived from heats of combustions of pure substances. As such, it is possible to calculate uncertainties that are not based on an empirical correlation derived from ultimate and HHV results extracted from the data in the USGS coal quality database.

Conversely, the proximate validation algorithm is based on an empirical correlation, albeit developed from the results of several independent coal quality databases (Swartzman, 1953; Swartzman and Tibbetts, 1955; Tibbetts and Montgomery, 1960; Tibbetts and others, 1978; Faurschou and others, 1982; Bonnell and others, 1983; Joint Coal Board and Queensland Coal Board, 1979; and CANSPEX, a proprietary database by Quality Associates International Ltd.). For this reason, the validation rating categories for proximate are set only as either ACCEPTABLE or SUSPECT. The summary for the proximate validation algorithm is shown in table 2. The proximate validation resulted in 6,659 of the 6,692 complete proximate datasets (99.51\%) passing with a rating of ACCEPTABLE. In a case where the proximate rating is SUSPECT and the ultimate and HHV rating is not INVESTIGATE, the most likely cause is a questionable volatile matter result. This is because moisture, ash yield, and volatile matter are used in the proximate validation. Moisture and ash yield are also used in the ultimate and HHV validation, but volatile matter is not used.

Two parameter columns labeled as Ultimate Validation and Proximate Validation (*Ultimate Validation* and *Proximate Validation* in table A2-1) were added to COALQUAL Version 3.0 (see appendix 2) to present the results of the validation process for all samples with sufficient data required by the validation algorithms. The validation results provide the user of the data an additional tool that can be used to help decide which data would be most appropriate for a particular use. For example, a sample with an INVESTIGATE rating for the HHV and ultimate validation may not be chosen for inclusion in the determination of apparent rank for a particular area, but the use of the trace element analyses for that same sample may be acceptable.

Table 2. Validation summary for the degree of agreement between the proximate analyses and the carbon and hydrogen values from the ultimate analyses. [Validated samples $(6,692)$ equals the total number of samples $(7,644)$ minus the number of incomplete samples (952), and refers to number of complete datasets. Percent $(\%)$ is of 6,692 .]

\begin{tabular}{clc}
\hline \multicolumn{2}{l}{ Proximate Validation } \\
\hline \multirow{2}{*}{ Number of samples } & Total & 7,644 \\
\cline { 2 - 3 } & Incomplete & 952 \\
\cline { 2 - 3 } & Validated & 6,692 \\
\hline \multirow{2}{*}{ Rating scale } & ACCEPTABLE & $99.51 \%$ \\
\cline { 2 - 3 } & SUSPECT & $0.49 \%$ \\
\hline
\end{tabular}

\section{Conclusion}

COALQUAL Version 3.0 represents the latest revision of the U.S. Geological Survey COALQUAL database. Unlike previous versions more than 95 percent of the data in the USCHEM database are included, while still maintaining the emphasis of full-bed data. All bench data used to calculate the full-bed data are included, as well as complete stratigraphic sections that include roof, floor, and parting samples. Improved georeferencing data makes it possible to easily determine which samples are related to a full-bed sample. The data qualifiers from USCHEM are included, as well as additional qualifiers, to more clearly explain how the data were collected and analyzed. Limitations on data usage are also more fully documented. 


\section{References Cited}

Annual Book of ASTM Standards, 2014, Section 5; Petroleum products, lubricants, and fossil fuels; v. 05.06; Gaseous fuels; coal and coke; Bioenergy and industrial chemicals from Biomass: West Conshohocken, Pa., ASTM International, 941 p.

Barnes, F.F., 1961, Coal fields of the United States; Alaska, sheet 2: U.S. Geological Survey map, scale 1:5,000,000.

Bonnell, G.W., Janke, L.C, and Romaniuk, A.S., 1983, Analysis directory of Canadian commercial coals; supplement no. 5: Canada Centre for Mineral and Energy Technology [CANMET] Report No. 84-01, 229 p.

Bragg, L.J., Oman, J.K., Tewalt, S.J., Oman, C.J., Rega, N.H., Washington, P.M., and Finkelman, R.B., 1994, U.S. Geological Survey coal quality (COALQUAL) database; Version 1.3: U.S. Geological Survey Open-File Report 94-206.

Bragg, L.J., Oman, J.K., Tewalt, S.J., Oman, C.L., Rega, N.H., Washington, P.M., and Finkelman, R.B., 1997, U.S. Geological Survey coal quality (COALQUAL) database; Version 2.0: U.S. Geological Survey Open-File Report 97-134, CD-ROM. [Also available at http://pubs.usgs.gov/of/1997/of97-134/.]

Faurschou, D.K., Bonnell, G.W., and Janke, L.C, 1982, Analysis directory of Canadian commercial coals; supplement no. 4: Canada Centre for Mineral and Energy Technology [CANMET] Report No. 82-13, 192 p.

Francis, H.E., and Lloyd, W.G., 1983, Predicting heating value from elemental composition: Journal of Coal Quality, v. 2, no. 2, p. $21-25$.

Hoeft, A.P., Luppens, J.A., Fuller, R.I, and Tucker, C.R., 1983, Coal analysis data interrelationships and crossplots: 3rd International Coal Testing Conference Proceedings, Lexington, Ky., 1983, Oct. 24-26, p. 95-100.

Joint Coal Board, and Queensland Coal Board, 1979, Australian black coals; abstract: Joint Coal Board (Australia and New South Wales) [and] Queensland Coal Board, Sydney, Australia, 48 p.

Luppens, J.A., Wilson, S.E., and Stanton, R.W., 1992, Manual on drilling, sampling, and analysis of coal: American Society for Testing and Materials (ASTM) International Manual Series MNL 11, Philadelphia, Penn., ASTM International, 61 p.

Swartzman, E., 1953, Analysis directory of Canadian coals: Canadian Department of Mines and Technical Surveys, Mines Branch Monograph 836, 204 p.

Swartzman, E., and Tibbetts, T.E., 1955, Analysis directory of Canadian commercial coals; supplement no. 1: Canadian Department of Mines and Technical Surveys, Mines Branch Monograph 850, 81 p.

Tibbetts, T.E., and Montgomery, W.J., 1960, Analysis directory of Canadian commercial coals; supplement no. 2: Canadian Department of Mines and Technical Surveys, Mines Branch Monograph 868, 56 p.

Tibbetts, T.E., Montgomery, W.J., and Faurschou, D.K., 1978, Analysis directory of Canadian commercial coals; supplement no. 3: Canada Centre for Mineral and Energy Technology [CANMET] Report No. 79-07, 81 p.

Trumbull, J.V.A., 1960, Coal fields of the United States, sheet 1: U.S. Geological Survey map, scale 1:5,000,000.

U.S. Geological Survey, 2011, National geologic map database; U.S. geologic names lexicon "Geolex": U.S. Department of the Interior, U.S. Geological Survey Web page, accessed June 1, 2012, at http://ngmdb.usgs.gov/Geolex/search.

Veness, Chris, 2010, Movable type scripts; Calculate distance, bearing and more between latitude/longitude points: Web page, accessed May 05, 2015, at http://www.movable-type.co.uk/scripts/latlong.html.

Wood, G.H., Jr., Kehn, T.M., Carter, M.D., and Culbertson, W.C., 1983, Coal resource classification system of the U.S. Geological Survey: U.S. Geological Survey Circular 891, 65 p. [Also available at http://pubs.er.usgs.gov/publication/cir891.] 


\section{Appendixes 1-7}

Appendix 1. Acceptance Rules for Upper Level Data

Appendix 2. Data Parameters

Appendix 3. Data Qualifiers

Appendix 4. Remnant Moisture Estimate Method

Appendix 5. Averaging Components Used to Calculate Upper Level Samples

Appendix 6. Strat Parameter

Appendix 7. Identification of Nearby Samples 


\section{Appendix 1. Acceptance Rules for Upper Level Data}

1. An Upper Level analysis in COALQUAL Version 3.0 is a sample or multiple samples that represents a full coal bed (full-bed samples) (from bottom to top), or meets the following criteria:

a. Channel samples must be composed of 100 percent of the coal bed thickness less any partings of material greater than 50 percent ash on a dry basis.

b. Core samples which are incomplete due to poor recovery are assumed to be representative if the percent core loss is less than 30 percent.

c. If any single parting is 12 inches or more, the coal bed is separated into two beds. If there are multiple partings 12 inches or more, 3 or more separate coal beds will be defined. Note that full-bed samples are only one criteria that defines Upper Level samples, but all Upper Level samples are full-bed samples.

d. Coal must be representative of a full coal bed, and not be oxidized or processed.

2. Samples may be rejected as representative of Upper Level samples for a variety of reasons. Rejected samples are included in miscellaneous data and can be searched by reason for rejection. The reasons for rejected samples include the following:

a. Grab samples: These are not considered representative of a full-bed or the mine from which they originated because of the typical high vertical inhomogeneity within a coal bed.

b. Tipple samples: These mined samples are collected at the coal processing site and are not representative of a single bed at a single location.

c. Run of mine samples: These mine samples are collected at the coal processing site and are not representative of a single bed at a single location (see tipple samples).

d. Pit samples: See grab samples.

e. Blend pile: These samples are taken from a pile of coal often from a specific bed, part of bed, or group of beds, but not collected in a manner to ensure a representative full-bed sample.

f. Stockpile: These samples are generally in a storage pile either at the mine, load-out facility, or power plant. In many cases, the exact origin of the coal is not known and they are not representative of a single bed. g. Feed coal: Coal being fed into a power plant, sometimes blended from several sources or taken from a stockpile.

h. Limestone sludge: Reacted limestone after it is used in the desulfurization process at a power plant.

i. Shot pile: Samples taken from coal that result from blasting at the coal face, which is commonly needed to break-up thick coal beds of the western United States before loading, and may not be representative of a single bed at a single location.

j. Dump: A pile of poor quality coal and (or) rock, often weathered, and not representative of mined coal or representative of a single bed at a single location.

k. Partial bed: An incomplete bed sample that is not representative of the full-bed primarily because of typical vertical inhomogeneity differences.

1. Partings greater than one foot: In some cases, a single sample is a physically composited sample of two coal intervals separated by a parting that is 1 foot or more thick. These samples are rejected because we define the 1-foot parting as two beds, and therefore the sample is not properly composited.

m. Rock: Samples with a dry ash content of greater than 50 percent are not coal samples (ASTM, 2014; see ASTM D121-09). They may represent partings, if less than 1 foot thick, or other related rock samples such as roof, floor, concretions, lenses, or other highash samples (also see tonsteins).

n. Drill cuttings, chip samples from a coal bed, an auger, or a churn drill: These coal samples from drilling operations are contaminated by non-coal rock fragments and are not considered representative of a full coal bed.

o. Less than 70 percent recovery: Drill core recovery less than 70 percent is not considered representative of a full coal bed.

p. Naturally burned or road cut burned: Burned samples that do not represent the original coal.

q. Materials that are not coal: These include materials such as drill additives, drill mud, drill fluid mix, fly ash, bottom ash, and slag.

r. Tonsteins: Thin layers of altered volcanic ash that can be used to determine the age of coal beds and sometimes used to help determine coal bed names. 
s. Weathered: We define a weathered sample as a sample that has a calorific value on a moistureand ash-free basis of $11,500 \mathrm{Btu} / \mathrm{lb}$ or less. Single full-bed samples that are weathered are rejected as non-representative samples. Weathered bench samples are considered as representative only if they are composited with other bench samples in the full-bed. The composited samples are not considered weathered.

t. Washed: Coal that has been cleaned typically by a gravity separation process where the denser material (rocks, high-ash coal particles, and minerals such as pyrite) are separated and removed from the coal.

u. Alkaline extracts: Low-rank coal samples that have been leached in a weak alkaline solution, which is believed to represent coal before contact with ground water. Because these samples are treated they are not equivalent to unleached samples throughout the database.

v. Density: A sample divided into a specific gravity separate, which is not representative of in-situ coal.

w. No location: If there is no location known, then it is automatically rejected as an Upper Level sample. 


\section{Appendix 2. Data Parameters}

Appendix 2 provides a description of all data parameters (table A2-1) used in COALQUAL Version 3.0. Parameter names surrounded by asterisks and in bold such as *Sample ID*, are new parameters to COALQUAL Version 3.0. Other parameters were also in COALQUAL Version 1.3 and Version 2.0, but may have been referred to differently than in Version 3.0, which is explained in the individual descriptions provided in table A2-1.

Table A2-1. Descriptions of data parameters used in COALOUAL Version 3.0.

[Abbreviations: ASTM, American Society for Testing and Materials; Btu, British thermal units; ID, identification number; INAA, Instrumental Neutron Activation Analysis. ASTM (2014) is the current reference for ASTM Annual Book of Standards. Database samples were analyzed over a period of years beginning in 1973, and the ASTM methods used were from the current ASTM Book of Standards at the time of sample analysis.]

\begin{tabular}{ll}
\hline \multicolumn{1}{c}{ Parameter name } & Description \\
\hline *Sample ID* & Unique sample identification number generated by combining the Lab ID (W for samples analyzed \\
in Washington D.C. or Reston, Virginia, or D for samples analyzed in Denver, Colorado) and the \\
sample number from COALQUAL Version 1.3 and Version 2.0. For full-bed samples that are \\
determined by mathematically compositing coal bench samples (according to rules in appendix \\
5) with duplicates and composites (if applicable), an A (for averaged) or a C (for composites) \\
is used. The number of samples used for the composited value is placed after the Lab ID and \\
before the sample number of the upper most bench coal sample. For example, DA2161315 is \\
a mathematically averaged sample of D161315 and D161316. For composited samples using \\
U.S. Geological Survey data only, the Sample ID may contain a decimal point and a number as \\
part of the sample number, for example W217379.1 (appendix 6; table A6-8). \\
Related to Sample ID of an Upper Level sample. Only used for lower level samples such a \\
bench samples, partings, or miscellaneous samples. Only Upper Level samples were shown in \\
COALQUAL Version 1.3 and Version 2.0. \\
Name of State where sample was collected. This parameter was STATE in COALQUAL Version 1.3 \\
and Version 2.0. \\
Name of county in State (or borough name in Alaska) where sample was collected. This parameter \\
was COUNTY in COALQUAL Version 1.3 and Version 2.0. \\
Latitude coordinate for point source location of coal sample (decimal degrees). This parameter was \\
LATITUDE in COALQUAL Version 1.3 and Version 2.0. The N/S (north/south) parameter was \\
eliminated because a positive number for latitude is understood to be north of the equator, and \\
therefore all values in this database are positive.
\end{tabular}

Longitude

Longitude coordinate for point source location of coal sample (decimal degrees). This parameter was LONGITUD in COALQUAL Version 1.3 and Version 2.0. The E/W (east/west) parameter was eliminated by converting all longitude data to a negative number because a negative number for longitude is understood to be west of the Greenwich meridian. All values in this database are negative, therefore all locations in the database are in the Western Hemisphere. The values in COALQUAL Version 1.3 and Version 2.0 were positive with a parameter "W" telling the user of the database that the numbers are in the Western Hemisphere.

Province

Coal province name from Wood and others (1983). This parameter was CPROVINC in COALQUAL Version 1.3 and Version 2.0.

Region

Coal region name from Wood and others (1983). This parameter was called CREGION in COALQUAL Version 1.3 and Version 2.0.

Field

Coal field name from Barnes (1961). Also see Trumbull (1960). This parameter was called CFIELD in COALQUAL Version 1.3 and Version 2.0.

District

District name. Depending on the state, this field may be mining districts, political divisions (such as townships or boroughs), or other subdivisions of the state. This parameter was called DISTRICT in COALQUAL Version 1.3 and Version 2.0.

Formation

Formation name. Stratigraphic formation name specified by the collector of the sample, usually a state geologist; or if collected by U.S. Geological Survey personnel see GEOLEX database (U.S. Geological Survey, 2011). This parameter was called CFORMATN in COALQUAL Version 1.3 and Version 2.0. 


\section{Table A2-1. Descriptions of data parameters used in COALQUAL Version 3.0.-Continued}

[Abbreviations: ASTM, American Society for Testing and Materials; Btu, British thermal units; ID, identification number; INAA, Instrumental Neutron Activation Analysis. ASTM (2014) is the current reference for ASTM Annual Book of Standards. Database samples were analyzed over a period of years beginning in 1973, and the ASTM methods used were from the current ASTM Book of Standards at the time of sample analysis.]

\begin{tabular}{|c|c|}
\hline Parameter name & Description \\
\hline Group & $\begin{array}{l}\text { Group name. Stratigraphic group name specified by the collector of the sample, usually a state } \\
\text { geologist; or if collected by U.S. Geological Survey see GEOLEX database (U.S. Geological } \\
\text { Survey, 2011). This parameter was called CGROUP in COALQUAL Version } 1.3 \text { and Version 2.0. }\end{array}$ \\
\hline Bed & $\begin{array}{l}\text { Bed name. Stratigraphic bed name specified by the collector of the sample, usually a state geologist; } \\
\text { or if collected by U.S. Geological Survey see GEOLEX database (U.S. Geological Survey, 2011). } \\
\text { This parameter was called CBED in COALQUAL Version } 1.3 \text { and Version 2.0. }\end{array}$ \\
\hline Coal Zone & $\begin{array}{l}\text { Relationship between non-coal material and the coal bed (parting, roof, or floor). This parameter } \\
\text { was CZONE in COALQUAL Version } 1.3 \text { and Version 2.0. }\end{array}$ \\
\hline Depth (in) & $\begin{array}{l}\text { Depth from the surface of the earth to the top of the sample, if the sample is part of a drill core. If } \\
\text { samples are not from a drill core, but the sample is a bench sample, then the depth is a measure } \\
\text { from the top of the uppermost bench to the top of the next sample in the benched series (depth is } \\
\text { measured in inches). This parameter was DEPTH in COALQUAL Version } 1.3 \text { and Version 2.0. }\end{array}$ \\
\hline System & $\begin{array}{l}\text { Designates a fundamental chronostratigraphic unit of the samples geologic age (U.S. Geological } \\
\text { Survey, 2011). This parameter was SYSTEM in COALQUAL Version } 1.3 \text { and Version 2.0. }\end{array}$ \\
\hline Series/Epoch & $\begin{array}{l}\text { Designates a fundamental chronostratigraphic/geochronologic unit of the samples geologic age; } \\
\text { the database provides either series or epoch (U.S. Geological Survey, 2011). This parameter was } \\
\text { SER_EPOC in COALQUAL Version } 1.3 \text { and Version 2.0. }\end{array}$ \\
\hline *Literature* & References to published data about a sample. \\
\hline Comments & $\begin{array}{l}\text { Miscellaneous comments. This parameter was COMMENTS in COALQUAL Version } 1.3 \text { and } \\
\text { Version 2.0. }\end{array}$ \\
\hline Map & $\begin{array}{l}\text { Usually the topographic quadrangle map name and series. Occasionally another type of map name } \\
\text { may be used along with the scale of the map. This parameter was MAP in COALQUAL Version } 1.3 \\
\text { and Version 2.0. }\end{array}$ \\
\hline Point Id & $\begin{array}{l}\text { Field number assigned by the collector or submitter of the sample. This parameter was POINTID in } \\
\text { COALQUAL Version } 1.3 \text { and Version 2.0. }\end{array}$ \\
\hline Submit Date & $\begin{array}{l}\text { The date the sample was confirmed by the analytical labs as having been submitted for analysis } \\
\text { (MM/DD/YYYY). This parameter was SUBDATE in COALQUAL Version } 1.3 \text { and Version 2.0. }\end{array}$ \\
\hline Sample Description & Complete sample description. \\
\hline Estimated Rank & $\begin{array}{l}\text { Rank identified by the collector in the field. Because the ash content was not known in the field } \\
\text { some rocks were identified as coals and some coals identified as rocks (see Sample Description } \\
\text { for complete description of sample). This parameter was ESTRANK in COALQUAL Version } 1.3 \\
\text { and Version 2.0. }\end{array}$ \\
\hline *Apparent Rank* & $\begin{array}{l}\text { Standard classification of coal by rank; ASTM D388 method determined based on ASTM (2014). } \\
\text { Calculations used ASTM data of samples to produce: (1) moist, mineral-matter-free Btu/lb, } \\
\text { (2) dry, mineral-matter-free fixed carbon, and (3) volatile matter values. }\end{array}$ \\
\hline Analytical Labs & $\begin{array}{l}\text { Laboratory where sample was analyzed. This parameter was LABCODE in COALQUAL } \\
\text { Version } 1.3 \text { and Version 2.0. }\end{array}$ \\
\hline
\end{tabular}




\section{Table A2-1. Descriptions of data parameters used in COALQUAL Version 3.0.-Continued}

[Abbreviations: ASTM, American Society for Testing and Materials; Btu, British thermal units; ID, identification number; INAA, Instrumental Neutron Activation Analysis. ASTM (2014) is the current reference for ASTM Annual Book of Standards. Database samples were analyzed over a period of years beginning in 1973, and the ASTM methods used were from the current ASTM Book of Standards at the time of sample analysis.]

\begin{tabular}{|c|c|}
\hline Parameter name & Description \\
\hline Sample Type & $\begin{array}{l}\text { Description of sampling and condition of sample. This parameter was SAMPTYPE in COALQUAL } \\
\text { Version } 1.3 \text { and Version 2.0. }\end{array}$ \\
\hline Values Represent & $\begin{array}{l}\text { Description of sampling and condition of sample. This parameter was VALREP in COALQUAL } \\
\text { Version } 1.3 \text { and Version 2.0. }\end{array}$ \\
\hline *Section* & Section number in township and range. \\
\hline *Quarters* & Quarters in section. \\
\hline *Tract* & Subdivision of location information. \\
\hline *Location Detail* & Details of location information. \\
\hline Moisture & $\begin{array}{l}\text { Moisture content (as-received basis) in percent by weight as determined by ASTM method D3173 } \\
\text { of ASTM (2014). This parameter is MOISTR in COALQUAL Version } 1.3 \text { and Version 2.0. }\end{array}$ \\
\hline *Moisture Q* & Qualifier (Q) for Moisture. \\
\hline Volatile Matter & $\begin{array}{l}\text { Volatile matter content in percent as determined by ASTM method D3175 of ASTM (2014). This } \\
\text { parameter was VOLMAT in COALQUAL Version } 1.3 \text { and Version 2.0. }\end{array}$ \\
\hline *Volatile Matter Q* & Qualifier for Volatile Matter. \\
\hline Fixed Carbon & $\begin{array}{l}\text { Fixed carbon content in percent as determined by ASTM method D3172 of ASTM (2014). This } \\
\text { parameter was FIXEDC in COALQUAL Version } 1.3 \text { and Version 2.0. }\end{array}$ \\
\hline *Proximate Validation* & $\begin{array}{l}\text { Validation rating for the proximate analysis. The proximate values of residual moisture, ash, and } \\
\text { volatile matter are used to calculate fixed carbon by the difference. Subtracting the fixed carbon } \\
\text { from the total carbon yields volatile carbon. Dividing this by } 12 \text { (atomic weight of carbon) gives } \\
\text { moles of volatile carbon. Dividing the volatile carbon by the moles of hydrogen gives moles of } \\
\text { volatile coal. This result should be between } 0.0 \text { and } 0.5 \text {. This validation serves to confirm the } \\
\text { reliability of the proximate results as well as the ultimate carbon and hydrogen. } \\
\text { 1. ACCEPTABLE: designation of results that fall in the range of } 0.0-0.5 \text {. } \\
\text { 2. SUSPECT: designation of results that do not fall in the range of } 0.0-0.5 \text {. } \\
\text { Incomplete data indicates that a sample is missing critical test results required to carry out a proper } \\
\text { validation. }\end{array}$ \\
\hline Hydrogen & $\begin{array}{l}\text { Hydrogen content in percent as determined by ASTM method D3178 of ASTM (2014). This } \\
\text { parameter was HYDRGN in COALQUAL Version } 1.3 \text { and Version 2.0; hydrogen in Version } 3.0 \\
\text { does not include hydrogen from moisture. }\end{array}$ \\
\hline *Hydrogen Q* & Qualifier for Hydrogen. \\
\hline Carbon & $\begin{array}{l}\text { Carbon content in percent as determined by ASTM method D3178 of ASTM (2014) in } \\
\text { U.S. Geological Survey labs or other labs. This parameter was CARBON in COALQUAL } \\
\text { Version } 1.3 \text { and Version 2.0. }\end{array}$ \\
\hline
\end{tabular}


Table A2-1. Descriptions of data parameters used in COALQUAL Version 3.0.-Continued

[Abbreviations: ASTM, American Society for Testing and Materials; Btu, British thermal units; ID, identification number; INAA, Instrumental Neutron Activation Analysis. ASTM (2014) is the current reference for ASTM Annual Book of Standards. Database samples were analyzed over a period of years beginning in 1973, and the ASTM methods used were from the current ASTM Book of Standards at the time of sample analysis.]

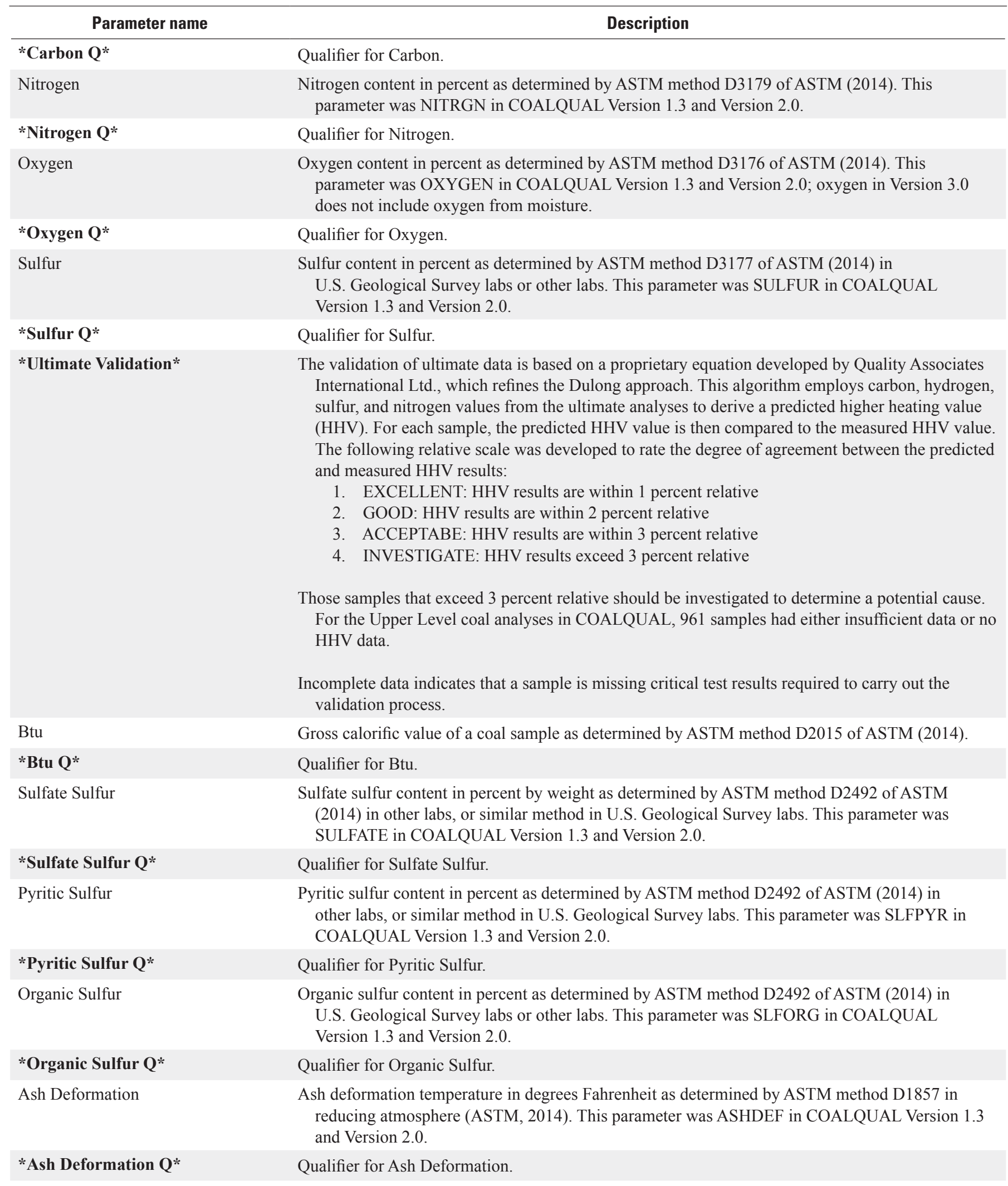


Table A2-1. Descriptions of data parameters used in COALQUAL Version 3.0.-Continued

[Abbreviations: ASTM, American Society for Testing and Materials; Btu, British thermal units; ID, identification number; INAA, Instrumental Neutron Activation Analysis. ASTM (2014) is the current reference for ASTM Annual Book of Standards. Database samples were analyzed over a period of years beginning in 1973, and the ASTM methods used were from the current ASTM Book of Standards at the time of sample analysis.]

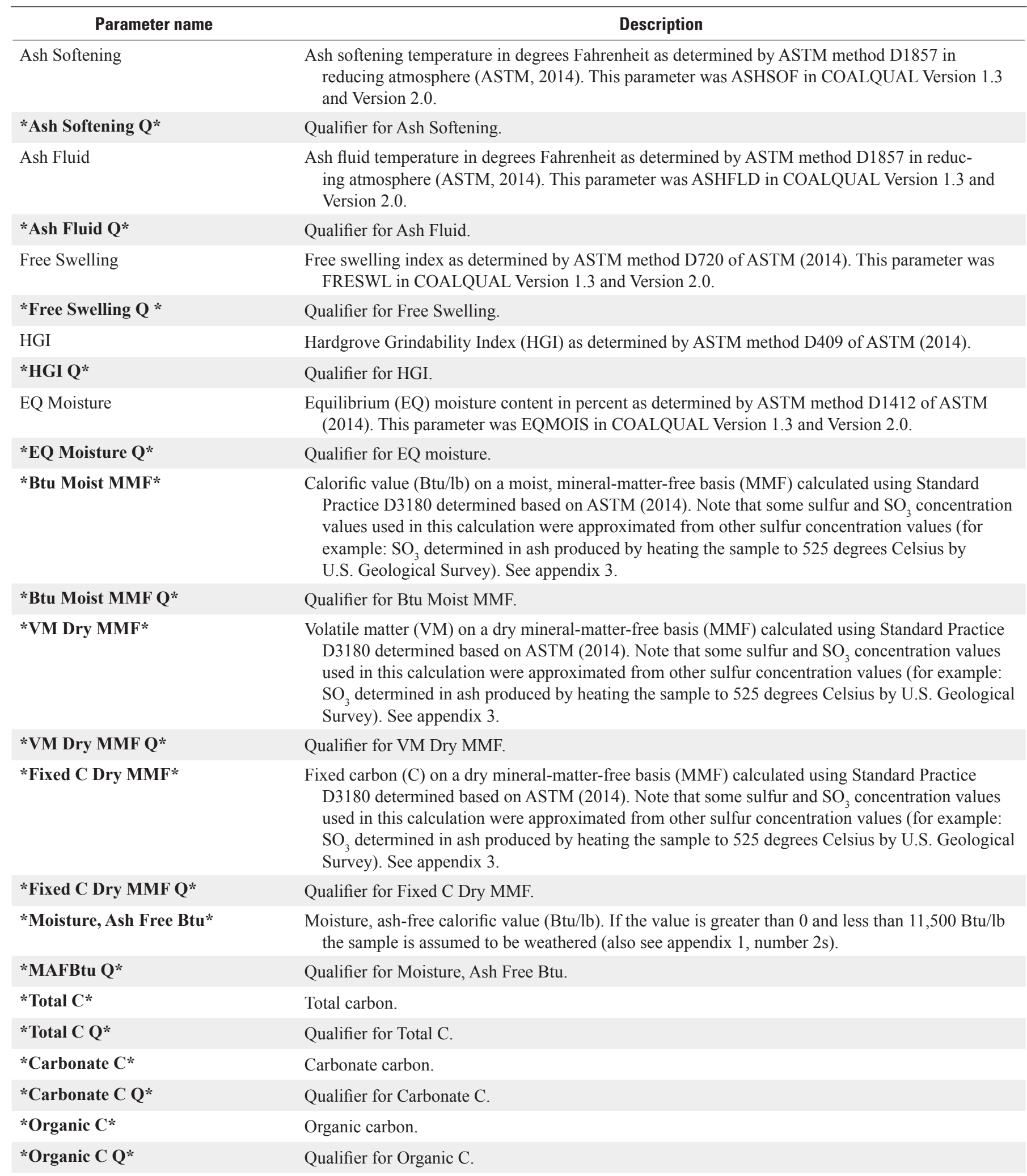




\section{Table A2-1. Descriptions of data parameters used in COALQUAL Version 3.0.-Continued}

[Abbreviations: ASTM, American Society for Testing and Materials; Btu, British thermal units; ID, identification number; INAA, Instrumental Neutron Activation Analysis. ASTM (2014) is the current reference for ASTM Annual Book of Standards. Database samples were analyzed over a period of years beginning in 1973, and the ASTM methods used were from the current ASTM Book of Standards at the time of sample analysis.]

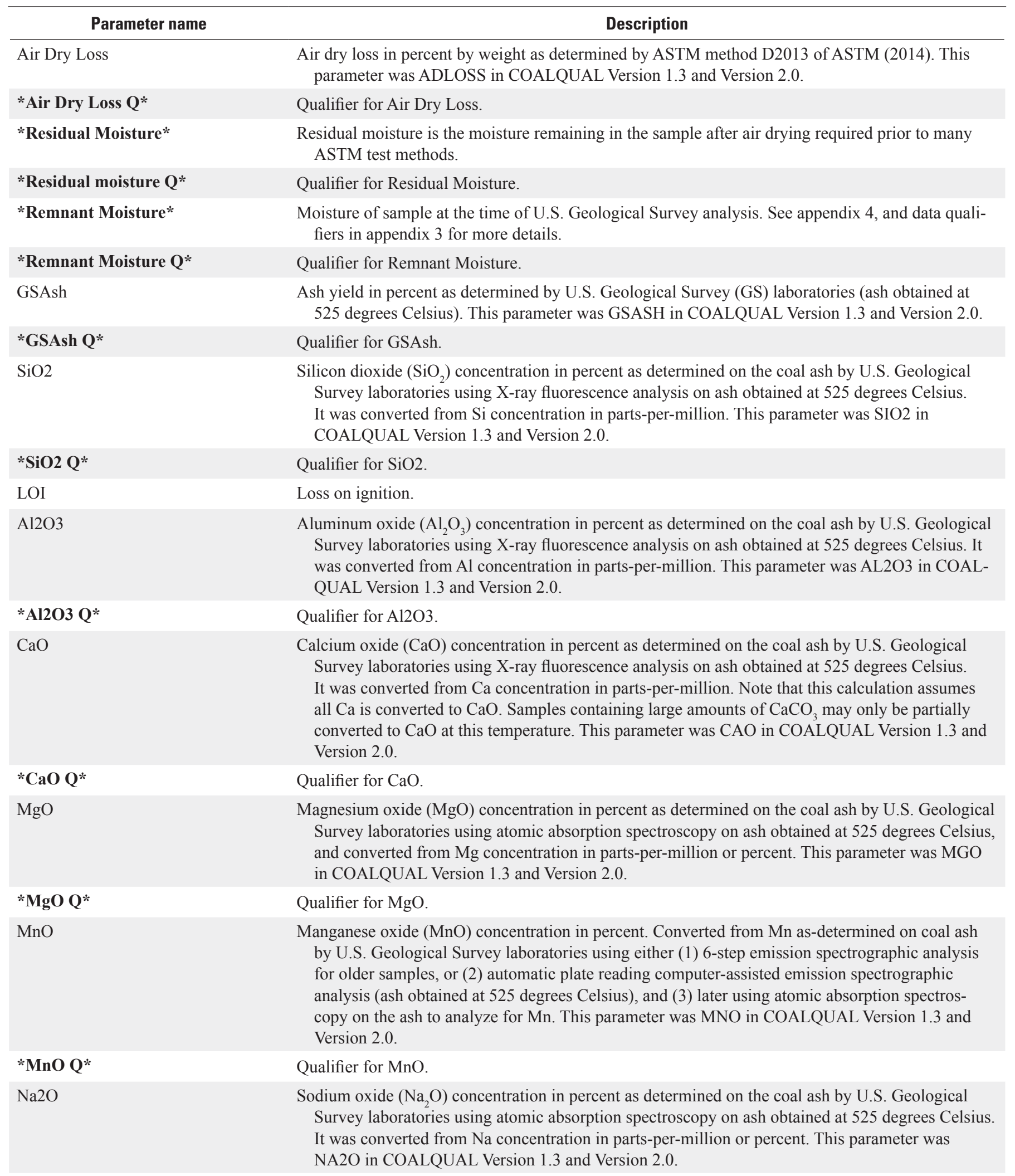




\section{Table A2-1. Descriptions of data parameters used in COALQUAL Version 3.0.-Continued}

[Abbreviations: ASTM, American Society for Testing and Materials; Btu, British thermal units; ID, identification number; INAA, Instrumental Neutron Activation Analysis. ASTM (2014) is the current reference for ASTM Annual Book of Standards. Database samples were analyzed over a period of years beginning in 1973, and the ASTM methods used were from the current ASTM Book of Standards at the time of sample analysis.]

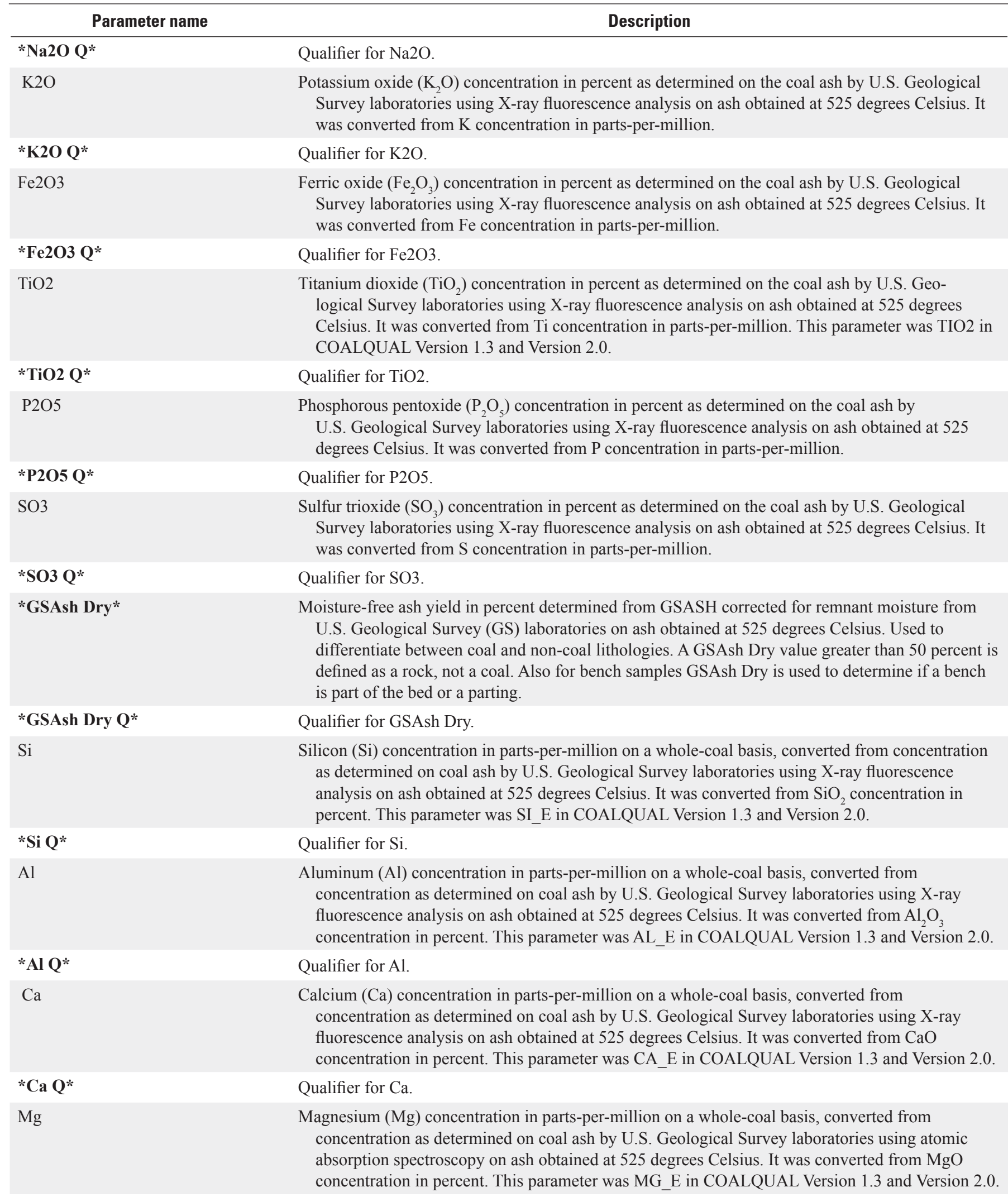




\section{Table A2-1. Descriptions of data parameters used in COALQUAL Version 3.0.-Continued}

[Abbreviations: ASTM, American Society for Testing and Materials; Btu, British thermal units; ID, identification number; INAA, Instrumental Neutron Activation Analysis. ASTM (2014) is the current reference for ASTM Annual Book of Standards. Database samples were analyzed over a period of years beginning in 1973, and the ASTM methods used were from the current ASTM Book of Standards at the time of sample analysis.]

\begin{tabular}{|c|c|}
\hline Parameter name & Description \\
\hline$* \operatorname{Mg} \mathbf{Q} *$ & Qualifier for Mg. \\
\hline $\mathrm{Na}$ & $\begin{array}{l}\text { Sodium }(\mathrm{Na}) \text { concentration in parts-per-million on a whole-coal basis, converted from concentration } \\
\text { as determined on coal ash by U.S. Geological Survey laboratories using atomic absorption } \\
\text { spectroscopy on ash obtained at } 525 \text { degrees Celsius. It was converted from } \mathrm{Na}_{2} \mathrm{O} \text { concentration } \\
\text { in percent. This parameter was NA_E in COALQUAL Version } 1.3 \text { and Version } 2.0 \text {. }\end{array}$ \\
\hline${ }^{*} \mathbf{N a} Q *$ & Qualifier for Na. \\
\hline K & $\begin{array}{l}\text { Potassium }(\mathrm{K}) \text { concentration in parts-per-million on a whole-coal basis, converted from } \\
\text { concentration as determined on coal ash by U.S. Geological Survey laboratories using X- } \\
\text { ray fluorescence analysis on ash obtained at } 525 \text { degrees Celsius. It was converted from } \mathrm{K}_{2} \mathrm{O} \\
\text { concentration in percent. This parameter was K_E in COALQUAL Version } 1.3 \text { and Version 2.0. }\end{array}$ \\
\hline$* \mathbf{K} \mathbf{Q}^{*}$ & Qualifier for K. \\
\hline $\mathrm{Fe}$ & $\begin{array}{l}\text { Iron (Fe) concentration in parts-per-million on a whole-coal basis, converted from concentration as } \\
\text { determined on coal ash by U.S. Geological Survey laboratories using X-ray fluorescence analysis } \\
\text { on ash obtained at } 525 \text { degrees Celsius. It was converted from } \mathrm{Fe}_{2} \mathrm{O}_{3} \text { concentration in percent. } \\
\text { This parameter was FE_E in COALQUAL Version } 1.3 \text { and Version } 2.0 \text {. }\end{array}$ \\
\hline *Fe Q* & Qualifier for Fe. \\
\hline $\mathrm{Ti}$ & $\begin{array}{l}\text { Titanium (Ti) concentration in parts-per-million on a whole-coal basis, converted from } \\
\text { concentration as determined on coal ash by U.S. Geological Survey laboratories using X-ray } \\
\text { fluorescence analysis on ash obtained at } 525 \text { degrees Celsius. It was converted from } \mathrm{TiO}_{2} \\
\text { concentration in percent. This parameter was TI_E in COALQUAL Version } 1.3 \text { and Version 2.0. }\end{array}$ \\
\hline$* \mathbf{T i} \mathbf{Q}^{*}$ & Qualifier for Ti. \\
\hline TS & $\begin{array}{l}\text { Total sulfur (TS) concentration in parts-per-million on a whole-coal basis, converted from } \\
\text { concentration as determined on coal ash by U.S. Geological Survey laboratories using X-ray } \\
\text { fluorescence analysis on ash obtained at } 525 \text { degrees Celsius. It was converted from } \mathrm{SO}_{3} \\
\text { concentration in percent. Note that this is not the standard ASTM sulfur method. }\end{array}$ \\
\hline$*$ TS Q* & Qualifier for TS. \\
\hline $\mathrm{Ag}$ & $\begin{array}{l}\text { Silver (Ag) concentration in parts-per-million on a whole-coal basis, converted from concentration } \\
\text { determined on coal ash by U.S. Geological Survey laboratories using either (1) 6-step emission } \\
\text { spectrographic analysis for older samples, or (2) automatic plate reading computer-assisted } \\
\text { emission spectrographic analysis (ash obtained at } 525 \text { degrees Celsius). This parameter was } \\
\text { AG_E in COALQUAL Version } 1.3 \text { and Version 2.0. }\end{array}$ \\
\hline$* \operatorname{Ag} Q^{*}$ & Qualifier for Ag. \\
\hline As & $\begin{array}{l}\text { Arsenic (As) concentration in parts-per-million as determined on a whole-coal basis by } \\
\text { U.S. Geological Survey laboratories using either (1) wet chemistry analysis for samples analyzed } \\
\text { in Denver, Colorado (where the first letter of Sample ID is D) before 1976, or (2) Instrumental } \\
\text { Neutron Activation Analysis (INAA) for samples analyzed in Reston, Virginia (where the first } \\
\text { letter of Sample ID is W) and for samples analyzed after } 1976 \text { in Denver. This parameter was } \\
\text { AS_E in COALQUAL Version } 1.3 \text { and Version 2.0. }\end{array}$ \\
\hline *As Q* & Qualifier for As. \\
\hline $\mathrm{Au}$ & $\begin{array}{l}\text { Gold }(\mathrm{Au}) \text { concentration in parts-per-million on a whole-coal basis, converted from concentration } \\
\text { determined on coal ash by U.S. Geological Survey laboratories using either (1) 6-step emission } \\
\text { spectrographic analysis for older samples, or (2) automatic plate reading computer-assisted } \\
\text { emission spectrographic analysis (ash obtained at } 525 \text { degrees Celsius). This parameter was } \\
\text { AU_E in COALQUAL Version } 1.3 \text { and Version 2.0. }\end{array}$ \\
\hline *Au Q* & Qualifier for Au. \\
\hline
\end{tabular}




\section{Table A2-1. Descriptions of data parameters used in COALQUAL Version 3.0.-Continued}

[Abbreviations: ASTM, American Society for Testing and Materials; Btu, British thermal units; ID, identification number; INAA, Instrumental Neutron Activation Analysis. ASTM (2014) is the current reference for ASTM Annual Book of Standards. Database samples were analyzed over a period of years beginning in 1973, and the ASTM methods used were from the current ASTM Book of Standards at the time of sample analysis.]

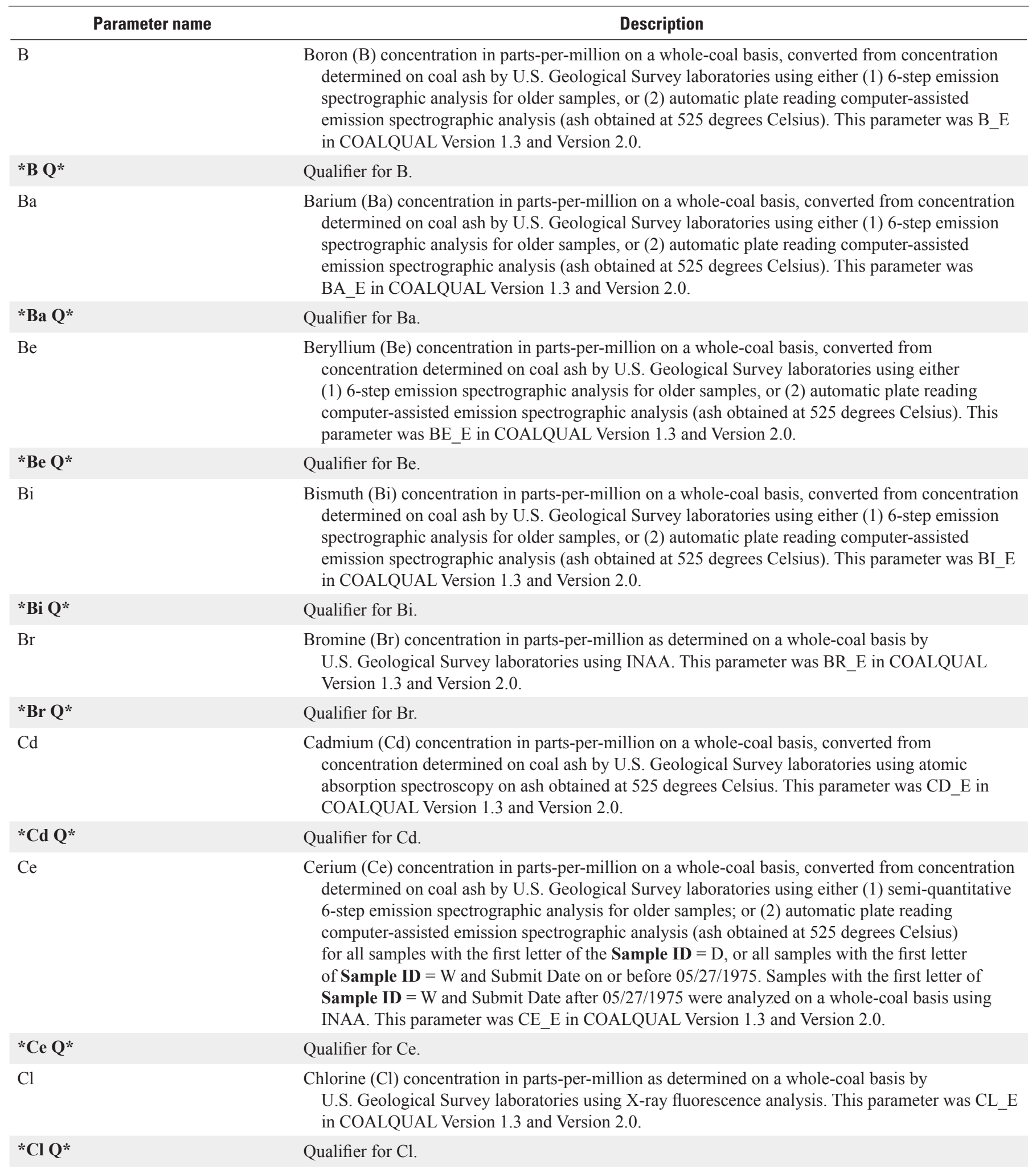




\section{Table A2-1. Descriptions of data parameters used in COALQUAL Version 3.0.-Continued}

[Abbreviations: ASTM, American Society for Testing and Materials; Btu, British thermal units; ID, identification number; INAA, Instrumental Neutron Activation Analysis. ASTM (2014) is the current reference for ASTM Annual Book of Standards. Database samples were analyzed over a period of years beginning in 1973, and the ASTM methods used were from the current ASTM Book of Standards at the time of sample analysis.]

\begin{tabular}{|c|c|}
\hline Parameter name & Description \\
\hline Co & $\begin{array}{l}\text { Cobalt (Co) concentration in parts-per-million on a whole-coal basis, converted from concentration } \\
\text { determined on coal ash by U.S. Geological Survey laboratories using either (1) semi-quantitative } \\
\text { 6-step emission spectrographic analysis for older samples; or (2) automatic plate reading } \\
\text { computer-assisted emission spectrographic analysis (ash obtained at } 525 \text { degrees Celsius) for } \\
\text { all samples with the first letter of Sample ID = D with Submit Date on or before 08/19/1976, } \\
\text { or all samples with the first letter of Sample ID = W and Submit Date on or before 05/27/1975. } \\
\text { Samples with the first letter of Sample ID = W and Submit Date after 05/27/1975, or the first } \\
\text { letter of Sample ID = D and Submit Date after 08/19/1976 were analyzed on a whole-coal basis } \\
\text { using INAA. This parameter was CO_E in COALQUAL Version } 1.3 \text { and Version } 2.0\end{array}$ \\
\hline *Co Q* & Qualifier for Co. \\
\hline $\mathrm{Cr}$ & $\begin{array}{l}\text { Chromium }(\mathrm{Cr}) \text { concentration in parts-per-million on a whole-coal basis, converted from concentration } \\
\text { determined on coal ash by U.S. Geological Survey laboratories using either (1) semi-quantitative } \\
\text { 6-step emission spectrographic analysis for older samples; or (2) automatic plate reading computer- } \\
\text { assisted emission spectrographic analysis (ash obtained at } 525 \text { degrees Celsius) for all samples with } \\
\text { the first letter of Sample ID = D with Submit Date } 08 / 19 / 1976 \text {, or all samples with the first letter of } \\
\text { Sample ID = W and Submit Date on or before } 05 / 27 / 1975 \text {. Samples with the first letter of Sample } \\
\text { ID =W and Submit Date after 05/27/1975, or the first letter of Sample ID = D and Submit Date } \\
\text { after 08/19/1976 were analyzed on a whole-coal basis using INAA. This parameter was CR_E in } \\
\text { COALQUAL Version } 1.3 \text { and Version } 2.0 \text {. }\end{array}$ \\
\hline${ }^{*} \mathrm{Cr} \mathbf{Q}^{*}$ & Qualifier for Cr. \\
\hline Cs & $\begin{array}{l}\text { Cesium (Cs) concentration in parts-per-million on a whole-coal basis, converted from concentration } \\
\text { determined on coal ash by U.S. Geological Survey laboratories using either (1) semi-quantitative } \\
\text { 6-step emission spectrographic analysis for older samples; or (2) automatic plate reading comput- } \\
\text { er-assisted emission spectrographic analysis (ash obtained at } 525 \text { degrees Celsius) for all samples } \\
\text { with the first letter of Sample ID = D, or all samples with the first letter of Sample ID = W and } \\
\text { Submit Date on or before } 05 / 27 / 1975 \text {. Samples with the first letter of Sample ID = W and Submit } \\
\text { Date after 05/27/1975 were analyzed on a whole-coal basis using INAA. This parameter was } \\
\text { CS_E in COALQUAL Version } 1.3 \text { and Version } 2.0 \text {. }\end{array}$ \\
\hline Dy & $\begin{array}{l}\text { Dysprosium (Dy) concentration in parts-per-million on a whole-coal basis, converted from } \\
\text { concentration determined on coal ash by U.S. Geological Survey laboratories using either } \\
\text { (1) 6-step emission spectrographic analysis for older samples, or (2) automatic plate reading } \\
\text { computer-assisted emission spectrographic analysis (ash obtained at } 525 \text { degrees Celsius). This } \\
\text { parameter was DY_E in COALQUAL Version } 1.3 \text { and Version 2.0. }\end{array}$ \\
\hline *Dy $\mathbf{Q} *$ & Qualifier for Dy. \\
\hline Er & $\begin{array}{l}\text { Erbium (Er) concentration in parts-per-million on a whole-coal basis, converted from concentration } \\
\text { determined on coal ash by U.S. Geological Survey laboratories using either (1) 6-step emission } \\
\text { spectrographic analysis for older samples, or (2) automatic plate reading computer-assisted } \\
\text { emission spectrographic analysis (ash obtained at } 525 \text { degrees Celsius). This parameter was ER_E } \\
\text { in COALQUAL Version } 1.3 \text { and Version 2.0. }\end{array}$ \\
\hline *Er Q* & Qualifier for Er. \\
\hline
\end{tabular}




\section{Table A2-1. Descriptions of data parameters used in COALQUAL Version 3.0.-Continued}

[Abbreviations: ASTM, American Society for Testing and Materials; Btu, British thermal units; ID, identification number; INAA, Instrumental Neutron Activation Analysis. ASTM (2014) is the current reference for ASTM Annual Book of Standards. Database samples were analyzed over a period of years beginning in 1973, and the ASTM methods used were from the current ASTM Book of Standards at the time of sample analysis.]

\begin{tabular}{|c|c|}
\hline Parameter name & Description \\
\hline $\mathrm{Eu}$ & $\begin{array}{l}\text { Europium (Eu) concentration in parts-per-million on a whole-coal basis, converted from concentration } \\
\text { determined on coal ash by U.S. Geological Survey laboratories using either (1) 6-step emission } \\
\text { spectrographic analysis for older samples; or (2) automatic plate reading computer-assisted emission } \\
\text { spectrographic analysis (ash obtained at } 525 \text { degrees Celsius) for all samples with the first letter of } \\
\text { Sample ID = D, or all samples with the first letter of Sample ID = W and Submit Date on or before } \\
\text { 05/27/1975. Samples with the first letter of Sample ID = W and Submit Date after 05/27/1975 were } \\
\text { analyzed on a whole-coal basis using INAA. This parameter was EU_E in COALQUAL Version } 1.3 \\
\text { and Version 2.0. }\end{array}$ \\
\hline
\end{tabular}

$\begin{array}{ll}\text { *Eu Q* } & \text { Qualifier for Eu. } \\ \text { F } & \text { Fluorine (F) concentration in parts-per-million as determined on a whole-coal by U.S. Geological } \\ & \text { Survey laboratories using an ion-selective electrode method. This parameter was F_E in COAL- } \\ & \text { QUAL Version } 1.3 \text { and Version 2.0. }\end{array}$

*F Q* Qualifier for F.

$\mathrm{Ga} \quad$ Gallium $(\mathrm{Ga})$ concentration in parts-per-million on a whole-coal basis, converted from concentration determined on coal ash by U.S. Geological Survey laboratories using either (1) 6-step emission spectrographic analysis for older samples, or (2) automatic plate reading computer-assisted emission spectrographic analysis (ash obtained at 525 degrees Celsius). This parameter was GA_E in COALQUAL Version 1.3 and Version 2.0.

$\begin{array}{ll}* G a Q^{*} & \text { Qualifier for Ga. } \\ \text { Gd } & \text { Gadolinium (Gd) concentration in parts-per-million on a whole-coal basis, converted from } \\ & \text { concentration determined on coal ash by U.S. Geological Survey laboratories using either } \\ & \text { (1) 6-step emission spectrographic analysis for older samples, or (2) automatic plate reading } \\ & \text { computer-assisted emission spectrographic analysis (ash obtained at } 525 \text { degrees Celsius). This } \\ \text { parameter was GD_E in COALQUAL Version } 1.3 \text { and Version 2.0. }\end{array}$

*Gd Q * Qualifier for Gd.

$\mathrm{Ge}$

Germanium $(\mathrm{Ge})$ concentration in parts-per-million on a whole-coal basis, converted from concentration determined on coal ash by U.S. Geological Survey laboratories using either (1) 6-step emission spectrographic analysis for older samples, or (2) automatic plate reading computer-assisted emission spectrographic analysis (ash obtained at 525 degrees Celsius). This parameter was GE_E in COALQUAL Version 1.3 and Version 2.0.

*Ge Q* Qualifier for Ge.

Hf

Hafnium (Hf) concentration in parts-per-million on a whole-coal basis, converted from concentration determined on coal ash by U.S. Geological Survey laboratories using either (1) 6-step emission spectrographic analysis for older samples; or (2) automatic plate reading computer-assisted emission spectrographic analysis (ash obtained at 525 degrees Celsius) for all samples with the first letter of Sample ID $=$ D, or all samples with the first letter of Sample ID $=\mathrm{W}$ and Submit Date on or before 05/27/1975. Samples with the first letter of Sample ID = W and Submit Date after 05/27/1975 were analyzed on a whole-coal basis using INAA. This parameter was HF_E in COALQUAL Version 1.3 and Version 2.0.
*Hf Q*
Qualifier for Hf.
$\mathrm{Hg}$
Mercury $(\mathrm{Hg})$ concentration in parts-per-million as determined on a whole-coal basis by U.S. Geological Survey laboratories using cold vapor atomic absorption spectroscopy. This parameter was HG_E in COALQUAL Version 1.3 and Version 2.0.

$\begin{array}{ll}* H g ~ Q * & \text { Qualifier for Hg. } \\ \text { Ho } & \text { Holmium (Ho) concentration in parts-per-million on a whole-coal basis, converted from } \\ & \text { concentration determined on coal ash by U.S. Geological Survey laboratories using either } \\ & \text { (1) 6-step emission spectrographic analysis for older samples, or (2) automatic plate reading } \\ & \text { computer-assisted emission spectrographic analysis (ash obtained at } 525 \text { degrees Celsius). This } \\ & \text { parameter was HO_E in COALQUAL Version } 1.3 \text { and Version 2.0. }\end{array}$




\section{Table A2-1. Descriptions of data parameters used in COALQUAL Version 3.0.-Continued}

[Abbreviations: ASTM, American Society for Testing and Materials; Btu, British thermal units; ID, identification number; INAA, Instrumental Neutron Activation Analysis. ASTM (2014) is the current reference for ASTM Annual Book of Standards. Database samples were analyzed over a period of years beginning in 1973, and the ASTM methods used were from the current ASTM Book of Standards at the time of sample analysis.]

\begin{tabular}{lc}
\hline \multicolumn{1}{c}{ Parameter name } & \multicolumn{1}{c}{ Description } \\
\hline *Ho Q* & Qualifier for Ho. \\
In & Indium (In) concentration in parts-per-million on a whole-coal basis, converted from concentration \\
& determined on coal ash by U.S. Geological Survey laboratories using either (1) 6-step emission \\
& spectrographic analysis for older samples, or (2) automatic plate reading computer-assisted \\
& emission spectrographic analysis (ash obtained at 525 degrees Celsius). This parameter was IN_E \\
& in COALQUAL Version 1.3 and Version 2.0.
\end{tabular}

*In $Q^{*}$

Ir

\section{*Ir Q*}

$\mathrm{La}$

\section{$* \mathbf{L a} \mathbf{Q} *$}

$\mathrm{Li}$

\section{${ }^{*} \mathbf{L i} \mathbf{Q}^{*}$}

$\mathrm{Lu}$

\section{*Lu Q* \\ $\mathrm{Mn}$}

\section{$* \operatorname{Mn} \mathbf{Q}^{*}$}

Mo
Qualifier for In.

Iridium (Ir) concentration in parts-per-million on a whole-coal basis, converted from concentration determined on coal ash by U.S. Geological Survey laboratories using either (1) 6-step emission spectrographic analysis for older samples, or (2) automatic plate reading computer-assisted emission spectrographic analysis (ash obtained at 525 degrees Celsius). This parameter was IR_E in COALQUAL Version 1.3 and Version 2.0.

Qualifier for Ir.

Lanthanum (La) concentration in parts-per-million on a whole-coal basis, converted from concentration determined on coal ash by U.S. Geological Survey laboratories using either (1) 6-step emission spectrographic analysis for older samples; or (2) automatic plate reading computer-assisted emission spectrographic analysis (ash obtained at 525 degrees Celsius) for all samples with the first letter of Sample ID $=$ D, or all samples with the first letter of Sample ID $=$ W and Submit Date on or before 05/27/1975. Samples with the first letter of Sample ID $=\mathrm{W}$ and Submit Date after 05/27/1975 were analyzed on a whole-coal basis using INAA. This parameter was LA_E in COALQUAL Version 1.3 and Version 2.0.

Qualifier for La.

Lithium (Li) concentration in parts-per-million on a whole-coal basis, converted from concentration determined on coal ash by U.S. Geological Survey laboratories using atomic absorption spectroscopy on ash obtained at 525 degrees Celsius. This parameter was LI_E in COALQUAL Version 1.3 and Version 2.0.

Qualifier for Li.

Lutetium ( $\mathrm{Lu}$ ) concentration in parts-per-million on a whole-coal basis, converted from concentration determined on coal ash by U.S. Geological Survey laboratories using either (1) 6-step emission spectrographic analysis for older samples; or (2) automatic plate reading computer-assisted emission spectrographic analysis (ash obtained at 525 degrees Celsius) for all samples with the first letter of Sample ID $=$ D, or all samples with the first letter of Sample ID $=\mathrm{W}$ and Submit Date on or before 05/27/1975. Samples with the first letter of Sample ID $=$ W and Submit Date after 05/27/1975 were analyzed on a whole-coal basis using INAA. This parameter was LU_E in COALQUAL Version 1.3 and Version 2.0.

Qualifier for $\mathrm{Lu}$.

Manganese (Mn) concentration in parts-per-million on a whole-coal basis, converted from concentration determined on coal ash by U.S. Geological Survey laboratories using either (1) 6-step emission spectrographic analysis for older samples, or (2) automatic plate reading computer-assisted emission spectrographic analysis (ash obtained at 525 degrees Celsius), and (3) later using atomic absorption spectroscopy on the ash. This parameter was MN_E in COALQUAL Version 1.3 and Version 2.0.

Qualifier for Mn.

Molybdenum (Mo) concentration in parts-per-million on a whole-coal basis, converted from concentration determined on coal ash by U.S. Geological Survey laboratories using either (1) 6-step emission spectrographic analysis for older samples, or (2) automatic plate reading computer-assisted emission spectrographic analysis (ash obtained at 525 degrees Celsius). This parameter was MO_E in COALQUAL Version 1.3 and Version 2.0. 


\section{Table A2-1. Descriptions of data parameters used in COALQUAL Version 3.0.-Continued}

[Abbreviations: ASTM, American Society for Testing and Materials; Btu, British thermal units; ID, identification number; INAA, Instrumental Neutron Activation Analysis. ASTM (2014) is the current reference for ASTM Annual Book of Standards. Database samples were analyzed over a period of years beginning in 1973, and the ASTM methods used were from the current ASTM Book of Standards at the time of sample analysis.]

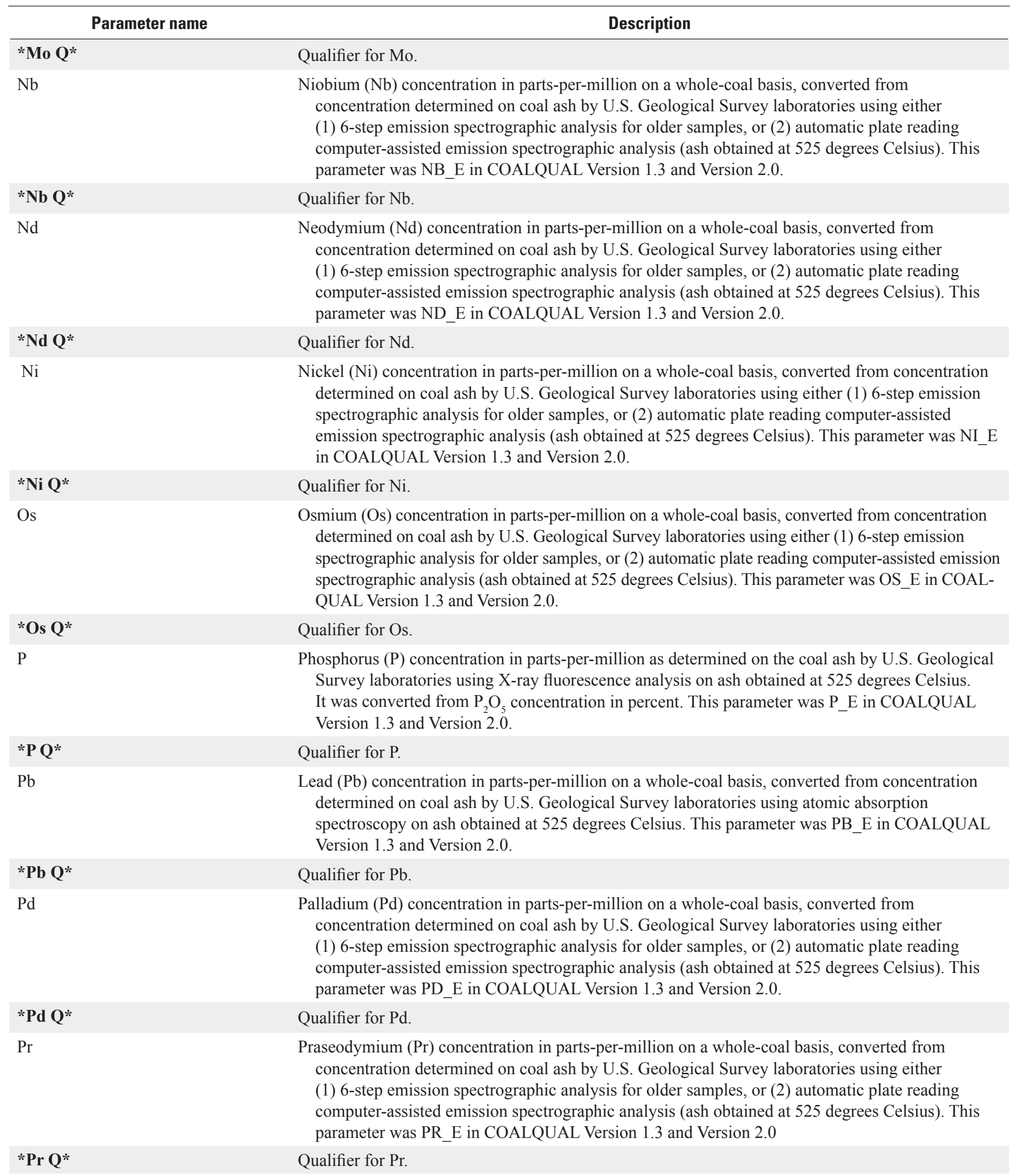




\section{Table A2-1. Descriptions of data parameters used in COALQUAL Version 3.0.-Continued}

[Abbreviations: ASTM, American Society for Testing and Materials; Btu, British thermal units; ID, identification number; INAA, Instrumental Neutron Activation Analysis. ASTM (2014) is the current reference for ASTM Annual Book of Standards. Database samples were analyzed over a period of years beginning in 1973, and the ASTM methods used were from the current ASTM Book of Standards at the time of sample analysis.]

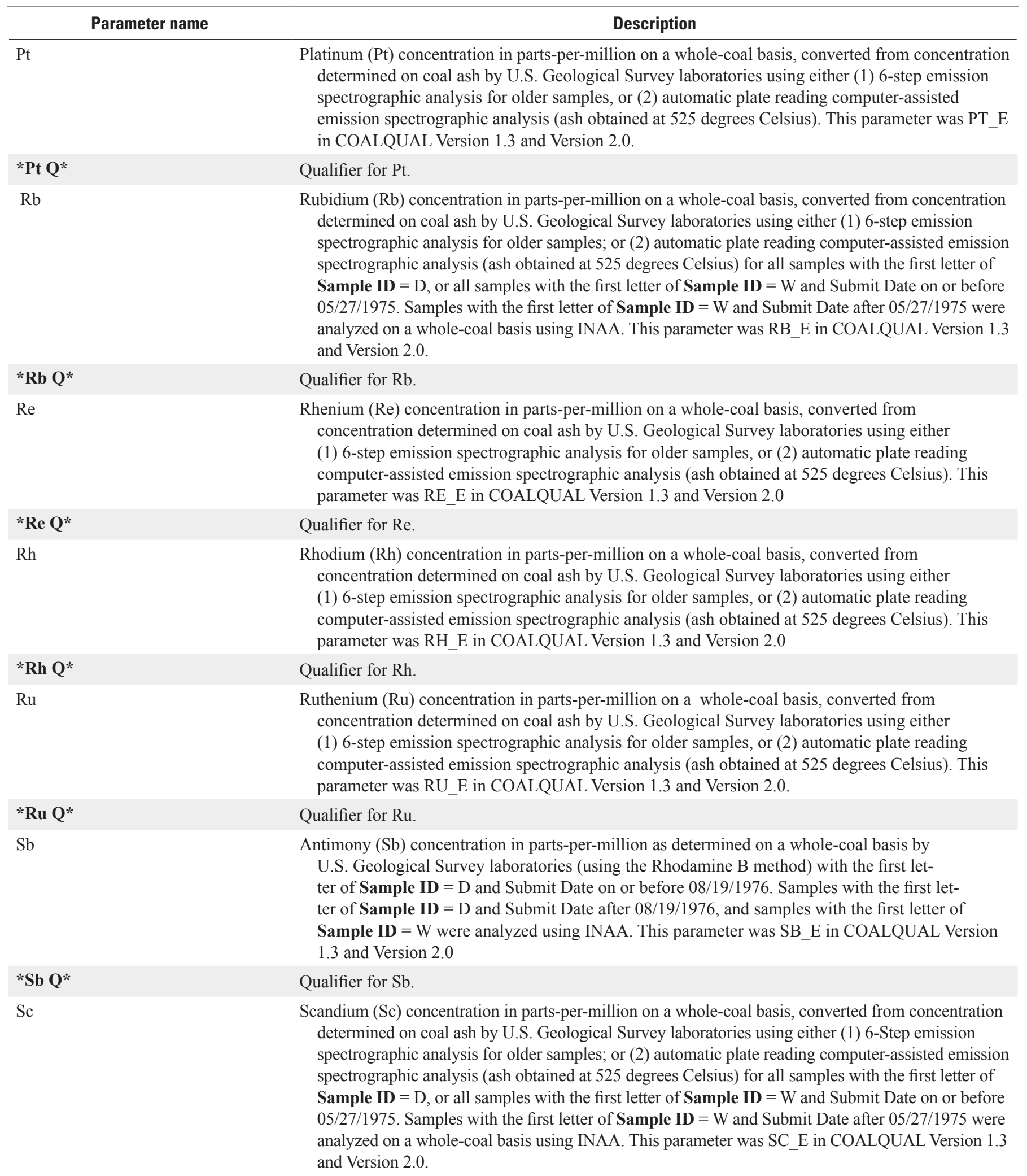




\section{Table A2-1. Descriptions of data parameters used in COALQUAL Version 3.0.-Continued}

[Abbreviations: ASTM, American Society for Testing and Materials; Btu, British thermal units; ID, identification number; INAA, Instrumental Neutron Activation Analysis. ASTM (2014) is the current reference for ASTM Annual Book of Standards. Database samples were analyzed over a period of years beginning in 1973, and the ASTM methods used were from the current ASTM Book of Standards at the time of sample analysis.]

\begin{tabular}{ll}
\hline \multicolumn{1}{c}{ Parameter name } & \multicolumn{1}{c}{ Description } \\
\hline *Sc Q* & Qualifier for Sc. \\
Se & Selenium (Se) concentration in parts-per-million as determined on a whole-coal basis by \\
& U.S. Geological Survey laboratories using X-ray fluorescence on all older samples with the first \\
& letter of Sample ID $=\mathrm{D}$ and Submit Date on or before $01 / 05 / 1978$, and all samples with the \\
& first letter of Sample ID $=\mathrm{W}$ and Submit Date on or before $06 / 01 / 1975$. Samples with the first \\
& letter of Sample ID $=\mathrm{D}$ and Submit Date after $01 / 05 / 1976$, and all samples with the first letter \\
& of Sample ID $=\mathrm{W}$ and Submit Date after $06 / 01 / 1976$ were analyzed on a whole-coal basis using \\
& INAA. This parameter was SE_E in COALQUAL Version 1.3 and Version 2.0.
\end{tabular}

*Se Q* Qualifier for Se.

$\mathrm{Sm}$

\section{$* \operatorname{Sm} Q *$}

$\mathrm{Sn}$

*Sn $\mathbf{Q} *$

$\mathrm{Sr}$

\section{*Sr Q*}

$\mathrm{Ta}$

\section{*Ta $Q *$}

$\mathrm{Tb}$
Samarium ( $\mathrm{Sm}$ ) concentration in parts-per-million on a whole-coal basis, converted from concentration determined on coal ash by U.S. Geological Survey laboratories using either (1) 6-step emission spectrographic analysis for older samples; or (2) automatic plate reading computer-assisted emission spectrographic analysis (ash obtained at 525 degrees Celsius) for all samples with the first letter of Sample ID $=$ D, or all samples with the first letter of Sample ID $=$ W and Submit Date on or before 07/01/1975. Samples with the first letter of Sample ID $=$ W and Submit Date after 07/01/1975 were analyzed on a whole-coal basis using INAA. This parameter was SM_E in COALQUAL Version 1.3 and Version 2.0.

Qualifier for Sm.

Tin (Sn) concentration in parts-per-million on a whole-coal basis, converted from concentration determined on coal ash by U.S. Geological Survey laboratories using either (1) 6-step emission spectrographic analysis for older samples, or (2) automatic plate reading computer-assisted emission spectrographic analysis (ash obtained at 525 degrees Celsius). This parameter was SN_E in COALQUAL Version 1.3 and Version 2.0.

Qualifier for Sn.

Strontium ( $\mathrm{Sr}$ ) concentration in parts-per-million on a whole-coal basis, converted from concentration determined on coal ash by U.S. Geological Survey laboratories using either (1) 6-step emission spectrographic analysis for older samples, or (2) automatic plate reading computer-assisted emission spectrographic analysis (ash obtained at 525 degrees Celsius). This parameter was SR_E in COALQUAL Version 1.3 and Version 2.0.

Qualifier for Sr.

Tantalum (Ta) concentration in parts-per-million on a whole-coal basis, converted from concentration determined on coal ash by U.S. Geological Survey laboratories using either (1) 6-step emission spectrographic analysis for older samples, or (2) automatic plate reading computer-assisted emission spectrographic analysis (ash obtained at 525 degrees Celsius) for all samples with the first letter of Sample ID $=$ D. Samples with the first letter of Sample ID $=$ W were analyzed on a whole-coal basis using INAA. This parameter was TA_E in COALQUAL Version 1.3 and Version 2.0.

Qualifier for Ta.

Terbium $(\mathrm{Tb})$ concentration in parts-per-million on a whole-coal basis, converted from concentration determined on coal ash by U.S. Geological Survey laboratories using either (1) 6-step emission spectrographic analysis for older samples; or (2) automatic plate reading computer-assisted emission spectrographic analysis (ash obtained at 525 degrees Celsius) for all samples with the first letter of Sample ID = D, or all samples with the first letter of Sample ID $=\mathrm{W}$ and Submit Date on or before 05/27/1975. Samples with the first letter of Sample ID $=$ W and Submit Date after 05/27/1975 were analyzed on a whole-coal basis using INAA. This parameter was TB_E in COALQUAL Version 1.3 and Version 2.0. 


\section{Table A2-1. Descriptions of data parameters used in COALQUAL Version 3.0.-Continued}

[Abbreviations: ASTM, American Society for Testing and Materials; Btu, British thermal units; ID, identification number; INAA, Instrumental Neutron Activation Analysis. ASTM (2014) is the current reference for ASTM Annual Book of Standards. Database samples were analyzed over a period of years beginning in 1973, and the ASTM methods used were from the current ASTM Book of Standards at the time of sample analysis.]

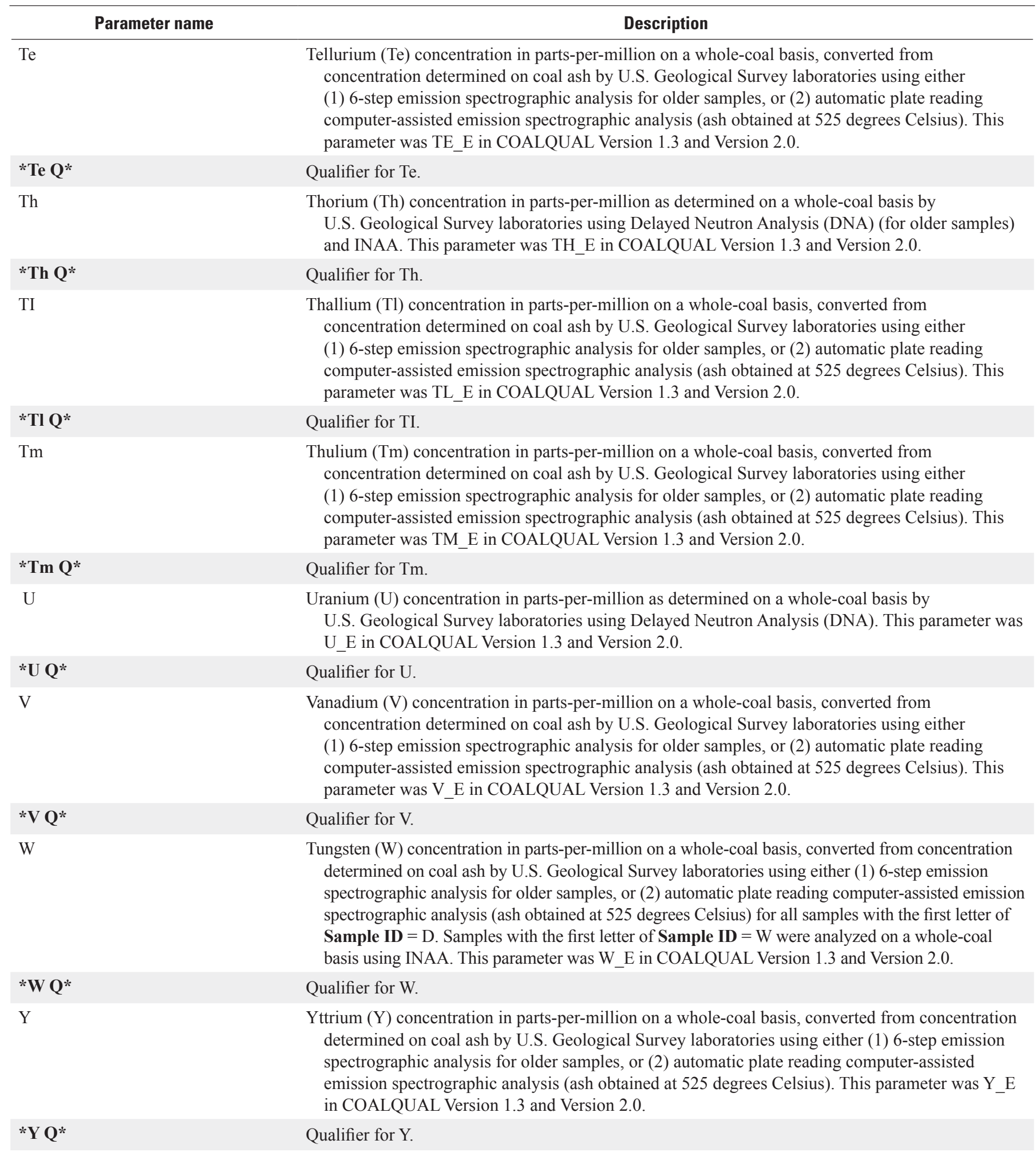


Table A2-1. Descriptions of data parameters used in COALQUAL Version 3.0.-Continued

[Abbreviations: ASTM, American Society for Testing and Materials; Btu, British thermal units; ID, identification number; INAA, Instrumental Neutron Activation Analysis. ASTM (2014) is the current reference for ASTM Annual Book of Standards. Database samples were analyzed over a period of years beginning in 1973, and the ASTM methods used were from the current ASTM Book of Standards at the time of sample analysis.]

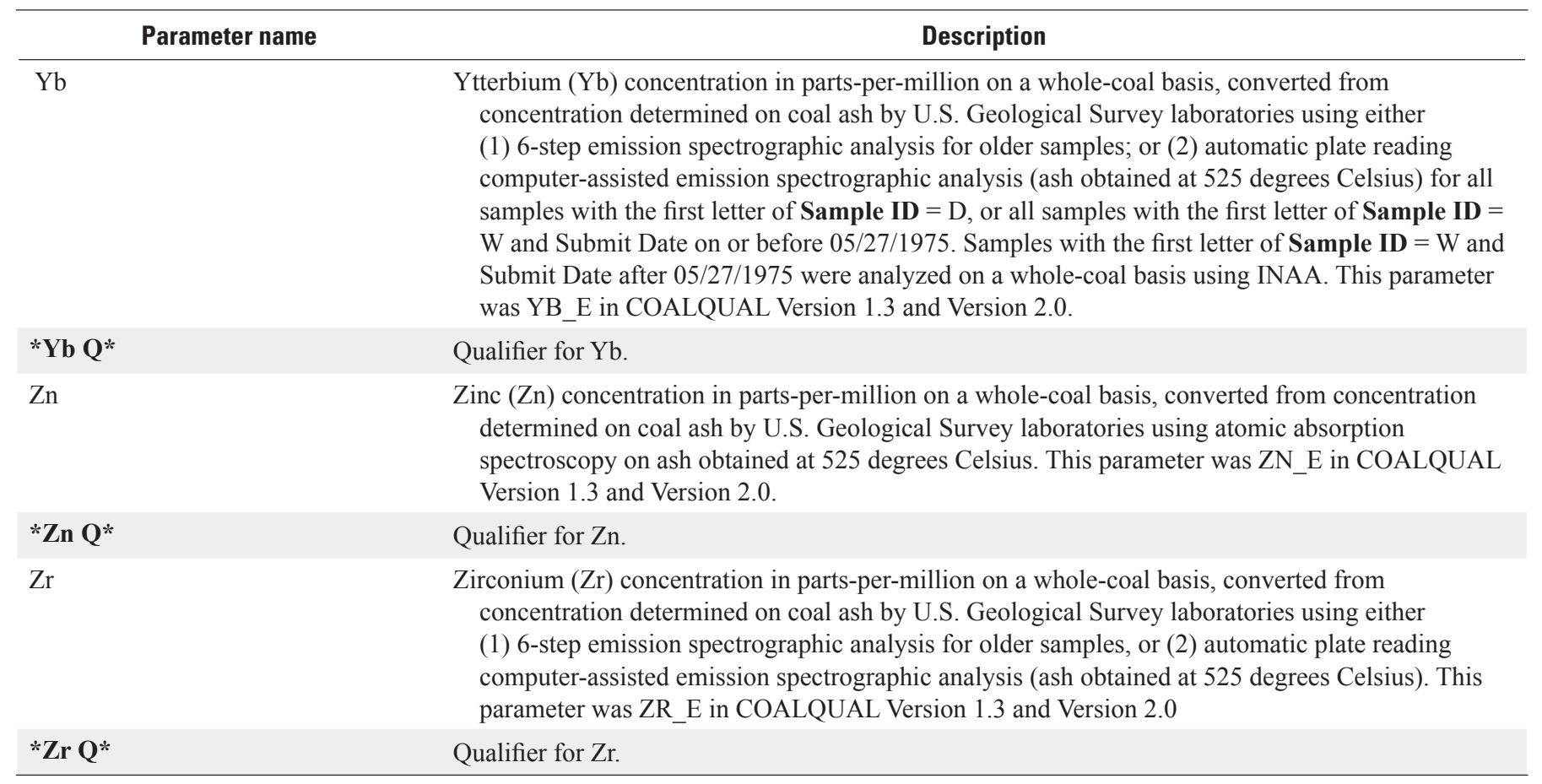




\section{Appendix 3. Data Qualifiers}

Appendix 3 provides a description of all data qualifiers used in COALQUAL Version 3.0 (table A3-1).

Table A3-1. Descriptions of data qualifiers used in COALQUAL Version. 3.0.

[Codes in uppercase indicate a value cannot be used as reported. Codes in lowercase mean a value has an error small enough not to affect the overall result, and therefore can be used as reported. Abbreviations: ASTM, American Society of Testing and Materials.]

\begin{tabular}{|c|c|}
\hline $\begin{array}{l}\text { Qualifier } \\
\text { Code }\end{array}$ & Description \\
\hline $\mathrm{L}$ & Less than. \\
\hline G & Greater than. \\
\hline $\mathrm{B}$ & Blank (no data) \\
\hline $\mathrm{N}$ & Not detected. \\
\hline $\mathrm{X}$ & Cannot be composited or averaged. \\
\hline a & See note in comment field in database. \\
\hline $\mathrm{m}$ & Miscellaneous \\
\hline a & Average \\
\hline e & Estimated moisture (derived directly from estimated rank). \\
\hline d & Computed chemical concentration using oxide data. \\
\hline $\mathrm{i}$ & Small or insignificant error for composited data. \\
\hline $\mathrm{u}$ & Analyzed by the U.S. Geological Survey. \\
\hline $\mathrm{s}$ & Originally reported as sulfur in the ash. \\
\hline $\mathrm{m}$ & More data available. \\
\hline $\mathrm{t}$ & $\begin{array}{l}\text { Total sulfur determined by the U.S. Geological Survey using methods similar to ASTM standard for sulfur D3177 (ASTM, } \\
\text { 2014). }\end{array}$ \\
\hline 1 & $\mathrm{SO}_{3}$ was estimated using loss of ignition (LOI). \\
\hline f & $\begin{array}{l}\mathrm{SO}_{3} \text { was estimated using the equation }(\mathrm{TS} / 0.4) \times\left(100 /(100-\mathrm{M}) \text {, where } \mathrm{TS} \text { is total sulfur, and } \mathrm{M} \text { is percent moisture; or } \mathrm{SO}_{3} \text { was }\right. \\
\text { estimated using the equation (dry sulfur } / 0.4) \text {. }\end{array}$ \\
\hline
\end{tabular}




\section{Appendix 4. Remnant Moisture Estimate Method}

\section{All Samples}

The discussion here in appendix 4 is based on a statistical evaluation of an independent data set with a large number of samples (with variable ranks) when both remnant and residual moisture values were both available. For high-rank coal, the statistical evaluation may tend to slightly overestimate the residual moisture. It may, in many cases, be an overestimation for low-rank coal, but reduces the likelihood of being incorrect on extremely high residual moisture values, and provides the lowest overall error for the database. Note that the samples used in the statistical evaluation are not part of the database.

If remnant moisture (as-determined moisture of sample at the time of USGS analysis) is known (or can be calculated), use it subject to the following conditions (also shown in table A4-1):

- If only residual moisture is known and is less than or equal to 4 percent, then residual moisture equals remnant moisture.

- If residual moisture is greater than 4 percent and less than or equal to 6 percent, subtract 4 percent from the residual moisture and divide by 2 , then add 4 percent to the result to determine the estimated remnant moisture.

- If residual moisture is greater than 6 percent and less than or equal to 7 percent, subtract 1 percent from the residual moisture to determine estimated remnant moisture.

- If residual moisture is greater than 7 percent and less than or equal to 9 percent, subtract 7 percent from the residual moisture and divide by 2 , then add 6 percent to the result to determine the estimated remnant moisture.

- If residual moisture is greater than 9 percent and less than or equal to 11 percent, subtract 2 percent from the residual moisture to determine the estimated remnant moisture.

- If residual moisture is greater than 11 percent and less than or equal to 13 percent, subtract 11 percent from the residual moisture and divide by 2 , then add 9 percent to the result to determine estimated remnant moisture.

- If residual moisture is greater than 13 percent and less than or equal to 19 percent, subtract 3 percent from the residual moisture to determine the estimated remnant moisture.

- If residual moisture is greater than 19 percent and less than or equal to 21 percent, subtract 19 percent from the residual moisture and divide by 2 , then add 16 percent to the result to determine estimated remnant moisture.

- If residual moisture is greater than 21 percent and less than or equal to 29 percent, subtract 4 percent from the residual moisture to determine the estimated remnant moisture.

- If residual moisture is greater than 29 percent and less than or equal to 31 percent, subtract 29 percent from the residual moisture and divide by 2 , then add 25 percent to the result to determine estimated remnant moisture.

- If residual moisture is greater than 31 percent, subtract 5 percent from the residual moisture value to determine the estimated remnant moisture.

Table A4-1. Input and criteria for estimating remnant moisture.

\begin{tabular}{lll}
\hline \multicolumn{1}{c}{$\begin{array}{c}\text { Residual moisture } \\
\text { (RM percent) }\end{array}$} & \multicolumn{1}{c}{ Formula used } & \multicolumn{1}{c}{$\begin{array}{c}\text { Estimated remnant moisture } \\
\text { (percent) }\end{array}$} \\
\hline$\leq 4$ & $\mathrm{RM}$ & $\leq 4$ \\
$>4$ and $\leq 6$ & $((\mathrm{RM}-4) / 2)+4$ & $>4$ and $\leq 5$ \\
$>6$ and $\leq 7$ & $\mathrm{RM}-1$ & $>5$ and $\leq 6$ \\
$>7$ and $\leq 9$ & $((\mathrm{RM}-7) / 2)+6$ & $>6$ and $\leq 7$ \\
$>9$ and $\leq 11$ & $\mathrm{RM}-2$ & $>7$ and $\leq 9$ \\
$>11$ and $\leq 13$ & $((\mathrm{RM}-11) / 2)+9$ & $>9$ and $\leq 10$ \\
$>13$ and $\leq 19$ & $\mathrm{RM}-3$ & $>10$ and $\leq 16$ \\
$>19$ and $\leq 21$ & $((\mathrm{RM}-19) / 2)+16$ & $>16$ and $\leq 17$ \\
$>21$ and $\leq 29$ & $\mathrm{RM}-4$ & $>17$ and $\leq 25$
\end{tabular}


Table A4-1. Input and criteria for estimating remnant moisture.-Continued

\begin{tabular}{lll}
\hline \multicolumn{1}{c}{$\begin{array}{c}\text { Residual moisture } \\
\text { (RM percent) }\end{array}$} & \multicolumn{1}{c}{ Formula used } & \multicolumn{1}{c}{$\begin{array}{c}\text { Estimated remnant moisture } \\
\text { (percent) }\end{array}$} \\
\hline$>29$ and $\leq 31$ & $((\mathrm{RM}-29 / 2)+25$ & $>25$ and $\leq 26$ \\
$>31$ & $\mathrm{RM}-5$ & $>26$ \\
\hline
\end{tabular}

Remnant moisture should be less than 30 percent (if higher than 30 percent it is rejected from the database). The residual moisture value should not be used if it exceeds the as-received moisture value. If the ash content is greater than 50 percent on a dry basis, it is defined as a non-coal sample.

\section{Semi-Anthracite and Anthracite Rank Samples}

If remnant moisture is known, use it as long as it is less than 10 percent. If residual moisture is not known, use 3 percent for residual moisture. A 1-sigma error (standard deviation) is less than 2 percent, although individual errors could be as high as 4 percent.

\section{Bituminous Rank Samples}

If remnant moisture is known, use it as long as it is less than 10 percent. If the residual moisture is high, it is likely that the rank is misidentified. If residual moisture is not known, use 4 percent for residual moisture. A 1-sigma error is less than 2 percent, although individual errors could be as high as 4 percent.

The tiered reduction of the residual moisture is based primarily on data for low-rank coals originally air dried in Somerset, Pennsylvania and analyzed in Denver, Colorado. The altitude and the drier climate in Denver can promote further drying of samples, even if sealed in polyethylene containers. Some samples that are not tightly sealed may experience even further drying, while samples with low as-received moisture content may already have experienced some drying, and may not experience additional moisture loss. Samples analyzed in Reston, Virginia, will often experience less drying than in Somerset or Denver due to higher humidity and lower elevation in Reston.

\section{Subbituminous Rank Samples}

If remnant moisture is known, use it as long as it is less than 20 percent. If residual moisture is not known, use 6 percent for residual moisture. A 1-sigma error is less than 5 percent error, although individual errors could be as high as 15 percent. Less than 0.6 percent of the samples with known residual moistures would have an error of 10 percent or more, if 6 percent residual moisture was used.

\section{Lignite Rank Samples}

If remnant moisture is known, use it as long as it is less than 30 percent. If residual moisture is not known, use 11 percent for residual moisture. A 1-sigma error is less than 5 percent error for the full dataset, although individual errors could be as high as 20 percent. Less than 0.5 percent of the samples with known residual moistures would have an error of 10 percent or more, if 11 percent residual moisture was used.

\section{Non-Coal and Unidentified Samples}

For samples with more than 50 percent ash or identified as non-coal samples, the moisture values are generally low. If there is no moisture information, assume 0 percent moisture. For samples that have no estimated rank and no ash content, or ash content less than 50 percent, use 10 percent estimated moisture to minimize overall errors. 


\section{Appendix 5. Averaging Components Used to Calculate Upper Level Samples}

When Upper Level samples represent multiple benches, duplicates, or triplicates (etc.), a weighted average ( $\left.\mathrm{AVE}_{\mathrm{wp}}\right)$ based on sample thickness and concentration (of any analyzed coal parameter) is used to determine the value of the weighted average using equation A5F1.

$$
\mathrm{AVE}_{\mathrm{wp}}=\sum \mathrm{V}_{\mathrm{ps}} \mathrm{T}_{\mathrm{s}} / \sum \mathrm{T}_{\mathrm{s}}
$$

Where $\mathrm{AVE}_{\mathrm{wp}}$ is the weighted (w) average (AVE) of a specific parameter/element (p). $\mathrm{V}_{\mathrm{ps}}$ is the value $\mathrm{V}$ for a specific parameter (p) in a given sample or subsample (s), and $\mathrm{T}_{\mathrm{s}}$ is the thickness (T) of the sample or subsample (s). In cases where only relative sample thickness was known, the ratios were used instead of the actual sample thickness. See appendix 2 for the format of sample identification (Sample ID) for these types of samples.

When subsamples are composited and some analytical results are qualified with "less than" (L) or "greater than" (G), the following adjustments are made to the average of analysis values.

1. When the average is calculated with values qualified with "e" (greater than), a qualifier code "G" is assigned to the average.

2. When the average is calculated with values qualified with "L" (less than), the following calculations are performed.

2.1. Compute the upper limit of the average values $\left(\mathrm{A}_{\mathrm{u}}\right)$ of all values by ignoring the "L" qualifier using equation $A 5 F 1$.

2.2. Compute the average lower limit of the average values $\left(A_{1}\right)$ by substituting " 0 " for the value with the " $L$ " qualifier.

2.3. Compute the average $\left(\mathrm{A}_{\mathrm{a}}\right)$ of the two averages $\left(\mathrm{A}_{\mathrm{u}}\right.$ and $\left.\mathrm{A}_{1}\right)$, obtained from the previous steps, 2.1 and 2.2, respectively.

2.4. The maximum absolute error $\left(\mathrm{E}_{\mathrm{am}}\right)$ is computed by calculating the difference between the two averages using equation A5F2.

$$
\mathrm{E}_{\mathrm{am}}=\mathrm{A}_{\mathrm{u}}-\mathrm{A}_{1}
$$

2.5. The maximum percent error $\left(\mathrm{E}_{\mathrm{pm}}\right)$ is computed as shown in equation $\mathrm{A} 5 \mathrm{~F} 3$.

$$
\mathrm{E}_{\mathrm{pm}}=\mathrm{E}_{\mathrm{am}} / \mathrm{A}_{\mathrm{a}} \times 100
$$

2.6. Finally, if the maximum percent error $\left(\mathrm{E}_{\mathrm{pm}}\right)$ is less than or equal to 5 percent, the error is considered to be insignificant with respect to the errors of analysis, and $\mathrm{A}_{\mathrm{a}}$ (average of $\mathrm{A}_{\mathrm{u}}$ and $\mathrm{A}_{\mathrm{L}}$ ) is taken as the final value. Otherwise, the largest value of $A_{u}$ is chosen to be the final value and is qualified with " $\mathrm{L}$ " (less than).

Note that an average cannot be computed if the subsample's analytical results contain both "less than" and "greater than" qualifiers.

3. There are three ways of determining the average value of a parameter when samples are physically composited as identified by the Strat data (given as *Strat* in table A2-1).

3.1. If there is only one value for a given parameter (or more generally a group of parameters such as all ASTM data or all USGS data) for all benches representing the entire bed sample, and all coal benches have been physically composited, those values become the average for that sample for that parameter.

3.2. If individual benches have data, and the data for all benches are physically composited, then the individual benches are combined using the weighted average (equation $\mathrm{A} 5 \mathrm{~F} 1$ ) and that result is further averaged with the composited data.

If only part of the full-bed is physically composited for some values as in 3.1, or all values as in 3.2, then the partial is treated as in 3.1 or 3.2 and further averaged with the remaining benches using equation A5F1. 


\section{Appendix 6. Strat Parameter}

The Strat parameter (*Strat* in table A2-1) is used to identify the stratigraphic (vertical) relationships of samples in the stratigraphic column for a single latitude and longitude location. This appendix explains the Strat parameter complexity, which is caused in part by the diversity of coal types and coal sequences throughout the United States, necessitating differing sample collection methods and sample types such as drill cores, bench sampling, roof, floor, parting, whole bed, and face-channel sampling.

Additionally, the diversity of objectives, differing laboratories, and frequent funding constraints added complexity to the sampling criteria. Samples were collected by many scientists from the USGS and State Geological Surveys for a variety of purposes. Furthermore, samples were prepared for analysis for major and trace elements in two different laboratories, and other coal parameters were analyzed by multiple contract laboratories. Uneven budget yearly funding occasionally forced reductions in the frequency of duplicate analyses, as well as the total number of samples analyzed. This required either the physical compositing of bench samples, or eliminating certain analytical procedures, or both. The following paragraphs describe the Strat parameter and provide examples for the different situations, beginning with less complex situations.

Single, Upper Level, full-bed samples at a given location are designated by their Sample ID followed by "-00." For example, the Strat parameter for the single, full-bed sample W196338 would be designated W196338-00. For individual samples that do not qualify as Upper Level samples at a given location, such as miscellaneous samples, there is no designation and the field is left blank, for example D163527.

When a full-bed Upper Level sample consists of multiple coal benches, sample values are mathematically composited according to the rules in appendix 5. The Sample ID for these contains an "A" for average and the number of benches averaged (see *Sample ID* in table A2-1), for example DA2161315. The Strat parameter notation for the composited average for the benches is D161315-00A:01,02. The sample number for the uppermost bench is assigned "-00A" for an Upper Level mathematically composited sample with the notation 01,02 denoting the individual benches (numbered from top to bottom) used for the composited analyses (table A6-1). The thickness of the Upper Level sample is the total thickness of the composited coal benches. Samples mathematically composited to produce the Upper Level and other non-coal samples such as partings, roof, and floor rocks etc., are organized in order from top to bottom. In most cases, the actual mean sea level elevations of the sample intervals are not known, but the thickness within a bed and relative stratigraphic positions are known. The samples of a sequence is identified by the Sample ID of the uppermost coal sample analyzed (full-bed or bench) followed by the sequence number. Table A6-1 presents a simple example of a stratigraphic sequence with two coal benches, and an Upper Level (full-bed) sample mathematically composited from the two bench samples.

Table A6-1. A simple example of a stratigraphic sequence with two coal benches, and an Upper Level sample mathematically composited from the two coal bench samples.

\begin{tabular}{llll}
\hline \multicolumn{1}{c}{ Sample ID } & \multicolumn{1}{c}{ Strat } & $\begin{array}{c}\text { Thickness } \\
\text { (feet) }\end{array}$ & \multicolumn{1}{c}{ Explanation } \\
\hline DA2161315 & D161315-00A:01,02 & 9.5 & $\begin{array}{c}\text { Upper Level (-00) sample mathematically obtained } \\
\text { by combining two coal benches (01 and 02) per } \\
\text { appendix 5. }\end{array}$ \\
D161315 & D161315-01 & 4.5 & $\begin{array}{l}\text { 1st coal bench (01). } \\
\text { D161316 }\end{array}$ \\
\hline
\end{tabular}

For more complex cases, additional letter designations are added for non-coal material associated with coal bed and coal materials (shown in table A6-2). These identifiers are defined by 5 different categories as follows:

1. Non-coal identifiers.

2. Stratigraphic identifiers.

3. Coal identifiers that identify samples used to create Upper Level samples. In many cases, these samples have no qualifier and are simply numbered as shown in table A6-1.

4. Coal identifiers that identify samples not used in Upper Level samples.

5. Identifiers containing both coal and non-coal material, which are not used in Upper Level samples. 
Examples of each of these cases are provided below. There is no single location where all of these designations exist, however there are many locations where multiple designations are used. Note that the comments in the last column of table A6-2 (Strat Type) are for descriptive purposes only and are not included the database.

Table A6-2. A list of additional letter designations used for non-coal material associated with coal bed and coal materials.

\begin{tabular}{|c|c|c|c|}
\hline Category & Identifier & Letter & Strat Type \\
\hline \multirow[t]{4}{*}{1} & \multirow[t]{4}{*}{ Non-coal identifiers } & $\mathrm{R}$ & Roof \\
\hline & & $\mathrm{F}$ & Floor \\
\hline & & $\mathrm{P}$ & Parting \\
\hline & & M & Miscellaneous (non-coal material) \\
\hline \multirow[t]{2}{*}{2} & \multirow[t]{2}{*}{ Stratigraphic identifiers } & SX & $\begin{array}{l}\text { Stratigraphic coal or non-coal sequence separated by at least a foot of rock material. } \\
\text { Where } \mathrm{X} \text { is the stratigraphic bed number, top to bottom. }\end{array}$ \\
\hline & & LX & $\begin{array}{l}\text { This indicator is used for stratigraphic sequences that are so similar in location there } \\
\text { is no apparent difference in the longitude and latitude, or used for stratigraphic } \\
\text { sequences that are at the same location but their relative stratigraphic positions are } \\
\text { unknown. } \mathrm{X} \text { is the number of samples at the location. }\end{array}$ \\
\hline \multirow[t]{4}{*}{3} & \multirow{4}{*}{$\begin{array}{l}\text { Coal identifiers that identify } \\
\text { samples used to create } \\
\text { Upper Level samples }\end{array}$} & $\mathrm{D}$ & Duplicate analysis \\
\hline & & $\mathrm{T}$ & ASTM data determined in physically composited samples. \\
\hline & & $\mathrm{U}$ & $\begin{array}{l}\text { U.S. Geological Survey data (major and trace elements) determined in physically } \\
\text { composited samples. }\end{array}$ \\
\hline & & $\mathrm{B}$ & $\begin{array}{l}\text { Both ASTM and U.S. Geological Survey data determined in physically composited } \\
\text { samples. }\end{array}$ \\
\hline 4 & $\begin{array}{l}\text { Coal identifiers that identify } \\
\text { samples not used in Upper } \\
\text { Level samples }\end{array}$ & $\mathrm{K}$ & Samples treated with alkaline solution of ammonium acetate. \\
\hline
\end{tabular}

In the first example (table A6-3), the Upper Level sample DA4169011 indicates the three benches starting with 169011 were analyzed in Denver (D) and were averaged (A) using rules in appendix 5. The Strat parameter D169011-00A:02,04,06 indicates that the Upper Level sample (-00) mathematically averages (A) coal benches 02,04 , and 06. Benches 01R (roof), 03P and 05P (partings), and 07F (floor) are not coal samples, and are therefore not part of the Upper Level sample. None of the partings have thickness of 1 foot or more, so all coal benches are part of the same Upper Level sample. 
Table A6-3. An example of a location with a coal sequence with roof, parting, and floor samples.

\begin{tabular}{llll}
\hline \multicolumn{1}{c}{ Sample ID } & \multicolumn{1}{c}{ Strat } & Thickness (feet) & \multicolumn{1}{c}{ Explanation } \\
\hline DA4169011 & D169011-00A:02,04,06 & 8.25 & $\begin{array}{l}\text { Upper Level sample (-00) mathematically obtained by } \\
\text { combining three coal benches (02, 04, and 06) per } \\
\text { appendix 5. }\end{array}$ \\
D169026 & D169011-01R & 0.5 & Roof (R) \\
D169011 & D169011-02 & 0.583 & 1st coal bench (02). \\
D169025 & D169011-03P & 0.25 & Parting (P) \\
D169010 & D169011-04 & 6.5 & 2nd coal bench (04). \\
D169024 & D169011-05P & 0.417 & Parting (P) \\
D169009 & D169011-06 & 1.17 & 3rd coal bench (06). \\
D169023 & D169011-07F & 0.25 & Floor (F) \\
\hline
\end{tabular}

The next example (table A6-4) has two different stratigraphic sequences at the same location. In this case, the upper most sequence (stratigraphic bed S1) has only one (01) miscellaneous (M) rock sample D165057, therefore the Strat parameter is D165057-01M-S1. This sequence has no Upper Level sample. The second stratigraphic sequence (S2) is below the first stratigraphic sequence, and contains a mathematically composited Upper Level sample DA2165058 from the combination of two coal bench samples D165058 and D165059 that are identified by the Strat parameters D165058-01-S2 and D165058-02-S2 in table A6-4.

Table A6-4. An example of a location with a miscellaneous rock sample and a coal sequence in two different stratigraphic sequences (S1 and S2).

\begin{tabular}{llcl}
\hline \multicolumn{1}{c}{ Sample ID } & \multicolumn{1}{c}{ Strat } & Thickness (feet) & Explanation \\
\hline D165057 & D165057-01M-S1 & 3.6 & $\begin{array}{c}\text { Miscellaneous (M) rock sample at least one foot above } \\
\text { D165058. }\end{array}$ \\
DA2165058 & D165058-00A:01,02-S2 & 9 & $\begin{array}{c}\text { Upper Level sample (-00) for stratigraphic bed 2 (S2) math- } \\
\text { ematically obtained by compositing two coal benches (01 } \\
\text { and 02) per appendix 5. }\end{array}$ \\
D165058 & D165058-01-S2 & & 1st coal bench (01). \\
D165059 & D165058-02-S2 & 4.5 & 2nd coal bench (02). \\
\hline
\end{tabular}

Table A6-5 illustrates stratigraphic sequences, which are at the same location but their relative stratigraphic positions are unknown, or samples that are known to be at a slightly different location, but the difference cannot be detected within the precision of the latitude and longitude in the database (usually within a few feet). The third example, shown in table A6-5, illustrates three different stratigraphic sequences with all samples so similar in location there is no apparent difference in latitude and longitude (locations L1, L2, and L3). The relative stratigraphic locations of the mathematically averaged (A) Upper Level samples for two of these locations are not known, location L1 (WA5232482) consists of four benches, and location L3 (WA2232573) consists of two benches. The Upper level single sample at location L2 (W232486) is below the bottom of location L1 by 6.4 feet. It should be noted that the " $\mathrm{L}$ " qualifier here is always followed by a number, and is different from the "L" qualifier in the elemental data, which always follows the number. 
Table A6-5. An example of three stratigraphic sequences that are at the same location, or so similar in location there is no apparent difference in latitude and longitude.

\begin{tabular}{llcl}
\hline \multicolumn{1}{c}{ Sample ID } & \multicolumn{1}{c}{ Strat } & $\begin{array}{c}\text { Thickness } \\
\text { (feet) }\end{array}$ & \multicolumn{1}{c}{ Explanation } \\
\hline WA5232482 & W232482-00A:01,02,03,04-L1 & 4.9 & Upper Level sample (-00) for location 1 (L1). \\
W232482 & W232482-01-L1 & 0.9 & 1st coal bench (01) location 1. \\
W232487 & W232482-02D-L1 & 0.9 & 2nd coal bench (02) location 1. \\
W232483 & W232482-02D-L1 & 0.9 & Duplicate (D) of 2nd coal bench \\
W232484 & W232482-03-L1 & 1.5 & 3rd coal bench (03) location 1. \\
W232485 & W232482-04-L1 & 1.6 & 4th coal bench (04) location 1. \\
W232486 & W232486-00-L2 & 1 & Upper Level (-00) single sample at location 2 (L2); \\
& W232573-00A:01,02-L3 & & this bed is below the bed at location 1. \\
WA2232573 & W232573-01-L3 & 1.2 & Upper Level (-00) sample for location 3 (L3). \\
W232573 & W232573-02-L3 & 0.6 & 1st coal bench (01) location 3. \\
W232572 & & 0.6 & 2nd coal bench (02) location 3. \\
\hline
\end{tabular}

Table A6-6 shows an example of a stratigraphic sequence that contains a duplicate (D) of the stratigraphic bed S1 and a floor (F) non-coal sample. Since the duplicates represent the same sample interval they both have the identifier "01D," after their Sample ID (of the Strat parameter designation). Note that if the sample was analyzed in Denver it has a letter "D" at the beginning of the sample ID, in contrast the "D" for duplicate sample is after the Strat parameter, for example D202213-1D. In this case, the sample number of the Upper Level sample is arbitrary since both samples represent the upper-most coal sample. These two samples are averaged (A) to obtain the mathematically composited Upper Level sample. If additional coal benches were in this sequence, the duplicates would have been averaged first, before being composited with the other coal bench samples according to the guidelines in appendix 5 .

Table A6-6. An example of a location with a stratigraphic sequence (S1) that contains both a duplicate and a floor sample.

\begin{tabular}{|c|c|c|c|}
\hline Sample ID & Strat & $\begin{array}{l}\text { Thickness } \\
\text { (feet) }\end{array}$ & Explanation \\
\hline WA2211942 & W211942-00A:01-S1 & 1.4 & $\begin{array}{l}\text { Upper Level sample (-00) obtained mathematically by combining } \\
\text { full-bed duplicates. }\end{array}$ \\
\hline W211942 & W211942-01D-S1 & 1.4 & Full-bed duplicate (D) of W211859. \\
\hline W211859 & W211942-01D-S1 & 1.4 & Full-bed duplicate (D) of W211942. \\
\hline W211939 & W211942-02F-S1 & 0.17 & Floor (F) sample for the full-bed samples above. \\
\hline
\end{tabular}

The next three examples show how composited data are handled in the database. There are three types of composited data. The first example relates to the two different types of analytical parameters determined in the database. Coal parameters analyzed in accordance with ASTM procedures are indicated by a "T" qualifier. In the second example, major and trace element data analyzed in USGS laboratories are indicated with a "U" qualifier. In the first two examples, depending on the original purpose for acquiring the data (and in some cases financial restrictions at the time of analysis), coal benches were physically composited to reduce the number of samples analyzed. In the third example, the composited sample was analyzed by both ASTM and USGS laboratories and indicated by the qualifier "B." In this last example, the data are treated as a single sample without a qualifier (since there are no data for individual benches). Otherwise, the physically composited benches are treated as duplicates of the whole sample, or of those benches composited. It should be noted that this qualifier is not the same as the qualifier "B" (blank) used to indicate blank data. Blank data is canceled by USGS and ASTM data fields, whereas the "B" (both) parameters is only found in Strat data.

The following paragraphs discuss each of these examples in more detail. The first, illustrates coal parameters (ASTM composite data) for three benches (table A6-7) at a location (L1). The "C" in the Sample ID number (DC3172313) represents composited data, the first "3" (after "DC") represents the number of coal benches composited, and the " $T$ " in the Strat parameter (D172313-00T:01,02,03-L1) represents ASTM composite data as discussed above. In this example, data for Sample ID DC3172313 contains only ASTM data. The coal bench samples contain only USGS data (major and trace element associated ash values, and so forth). The Upper Level sample (DA3172313) composites the coal bench samples according to appendix 5 for 
USGS data and ASTM data. Additional locations (L2 and L3) are not included in table A6-7 because they do not contain new information.

Table A6-7. An example of a location with ASTM composite data and three bench samples without ASTM data. The table includes only one of three locations found in the database at the associated latitude and longitude.

\begin{tabular}{llcc}
\hline \multicolumn{1}{c}{ Sample ID } & \multicolumn{1}{c}{ Strat } & Thickness (feet) & Explanation \\
\hline DA3172313 & D172313-00A:01,02,03-L1 & 64.8 & $\begin{array}{c}\text { Upper Level sample (-00) obtained by using the physically } \\
\text { composited ASTM data (DC3172313) and compositing } \\
\text { the USGS data from the three benches from location 1 } \\
\text { (L1). }\end{array}$ \\
DC3172313 & D172313-00T:01,02,03-L1 & 64.8 & $\begin{array}{c}\text { Physically composited sample of three bench samples (01, } \\
\text { 02, and 03) analyzed for ASTM parameters only (T). }\end{array}$ \\
D172313 & D172313-01-L1 & 21.6 & 1st coal bench (01) analyzed for USGS parameters only. \\
D172314 & D172313-02-L1 & 21.6 & 2nd coal bench (02) analyzed for USGS parameters only. \\
D172315 & D172313-03-L1 & 21.6 & 3rd coal bench (03) analyzed for USGS parameters only. \\
\hline
\end{tabular}

The next example (table A6-8) shows USGS data were analyzed for a sample physically composited from two coal benches. In this case, the full-bed sample W217379 was the only sample analyzed for major and trace elements. This sample was composited from two bench samples for which ASTM data were obtained. Because these samples had no USGS sample numbers, and all data in this database use USGS sample numbers, a decimal identification was attached to each bench resulting in Sample IDs W217379.1 and W217379.2. The Strat parameter for sample W217379 is W217379.1-00U:01,02-S4, because it is a USGS composite (U) of coal benches W217379.1 and W217379.2, "01" and "02," respectively. The Upper Level sample WA3217379.1 contains the USGS data from W217379 and the mathematically averaged ASTM data from W217379.1 and W217379.2. The stratigraphic sequence (S4) is one of five stratigraphic sequences sampled at this location; the other four sequences contained no new information, and therefore are not described in table A6-8.

Table A6-8. An example of a stratigraphic sequence (S4) at a location with U.S. Geological Survey (USGS) composite data and two bench samples without USGS data. Only one of five stratigraphic sequences from the database is included in the table.

\begin{tabular}{|c|c|c|c|}
\hline W217379 & W217379.1-00U:01,02-S4 & 1.75 & $\begin{array}{l}\text { Physically composited sample of the two coal benches } \\
(01 \text { and } 02) \text { analyzed for USGS parameters only (U). }\end{array}$ \\
\hline W217379.1 & W217379.1-01-S4 & 1.35 & $\begin{array}{l}\text { 1st coal bench sample (01) analyzed for ASTM param- } \\
\text { eters only. }\end{array}$ \\
\hline WA3217379.1 & W217379.1-00A:01,02-S4 & 1.75 & $\begin{array}{l}\text { Upper Level sample }(-00) \text { mathematically composited } \\
\text { using USGS data from the two coal bench samples } \\
\text { (01 and } 02) \text { and the physically composited sample. }\end{array}$ \\
\hline
\end{tabular}

The next example in table A6-9 shows a physically composited sample representing a full coal bed. Unlike the previous two examples, where all parameters were analyzed in all samples, the ASTM data were analyzed in a separate physically composited sample, which was considered to be essentially a duplicate of the mathematically averaged sample. The Upper Level sample WA4202051 is a combination of the mathematically composited data for benches 02,03 , and 04 containing USGS data, and the physically composited sample W202050 containing the ASTM data. 
Table A6-9. An example of a location with an Upper Level physically composited sample using both ASTM and U.S. Geological Survey (USGS) data, a roof, three coal benches, and a floor.

\begin{tabular}{llcl}
\hline Sample ID & \multicolumn{1}{c}{ Strat } & $\begin{array}{c}\text { Thickness } \\
\text { (feet) }\end{array}$ & \multicolumn{1}{c}{ Explanation } \\
\hline WA4202051 & $\begin{array}{c}\text { W202051- } \\
\text { 00A:02,03,04 }\end{array}$ & 3.83 & $\begin{array}{c}\text { Upper Level sample (-00) mathematically obtained by combining three coal } \\
\text { benches (02, 03, and 04) and the Upper Level composite per appendix 5. }\end{array}$ \\
W202050 & $\begin{array}{c}\text { W202051- } \\
\text { 00B:02,03,04 }\end{array}$ & 3.83 & $\begin{array}{c}\text { Physical composite of the three coal benches (02, 03, and 04) analyzed for } \\
\text { ASTM data only. }\end{array}$ \\
W202054 & W202051-01R & 0.33 & $\begin{array}{l}\text { Roof (R) } \\
\text { W202051 }\end{array}$ W202051-02 \\
W202052 & W202051-03 & 1.75 & 1st coal bench (02) analyzed for USGS data only. \\
W202053 & W202051-04 & 0.16 & 2nd coal bench (03) analyzed for USGS data only. \\
W202055 & W202051-05F & 0.17 & 3rd coal bench (04) analyzed for USGS data only. \\
\hline
\end{tabular}

Table A6-10 shows an example of two samples at two stratigraphic sequences treated with alkaline solution of ammonium acetate (identified with "K" after the sample number). The ammonium acetate solution removes elements attached to low-rank coal at ion exchangeable sites by groundwater. Since only a few samples of this kind are included in the database, they are not included in the Upper Level samples. In this example, the untreated sample splits are the Upper Level samples.

Table A6-10. An example of a location with two single Upper Level samples with splits treated with alkaline solution (K). The two Upper Level samples are separated by rock material by more than a foot forming two stratigraphic levels and an additional coal sequence at a third stratigraphic level.

\begin{tabular}{|c|c|c|c|}
\hline Sample ID & Strat & Thickness (feet) & Explanation \\
\hline D165598 & D165598-00-S1 & 6 & $\begin{array}{l}\text { Single Upper Level sample (-00) above S2 and S3 samples } \\
\text { (stratigraphic levels } 2 \text { and 3). }\end{array}$ \\
\hline D166369 & D165598-01K-S1 & 6 & Alkaline solution $(\mathrm{K})$ treated split of D165598. \\
\hline D165599 & D165599-00-S2 & 4.6 & $\begin{array}{l}\text { Single Upper Level sample (-00) above S3 and below S1 samples } \\
\text { (stratigraphic level 1). }\end{array}$ \\
\hline D166370 & D165599-01K-S2 & 4.6 & Alkaline solution $(\mathrm{K})$ treated split of D165599. \\
\hline DA2 165600 & D165600-00A:01,02-S3 & 15.9 & $\begin{array}{l}\text { Stratigraphic level } 3 \text { (S3) mathematically obtained by combining two } \\
\text { coal benches ( } 01 \text { and } 02) \text { as per appendix } 5 \text {. }\end{array}$ \\
\hline D165600 & D165600-01-S3 & 10 & 1 st coal bench $(01)$ for $\mathrm{S} 3$. \\
\hline D165601 & D165600-02-S3 & 5.9 & 2nd coal bench (02) for S3. \\
\hline
\end{tabular}

Table A6-11 shows an example of a location with a single sample. The Strat parameter includes an "I" for informational sample. The sample contains non-coal material (parting), and therefore it is not an Upper Level sample.

Table A6-11. An example of a location with an informational sample (I) that contains a parting (P).

\begin{tabular}{llll}
\hline Sample ID & Strat & $\begin{array}{c}\text { Thickness } \\
\text { (feet) }\end{array}$ & Explanation \\
\hline W202073 & W202073-00I:01,02P,03 & 36 & Not an Upper Level sample because it includes a parting (P). \\
\hline
\end{tabular}

Table A6-12 shows an example where a parting (P) is removed from a sample and the coal material from above and below the parting is recomposited to form a new sample D169253 (coal material). The parting was analyzed separately as D169264. 
Table A6-12. An example of a location with a stratigraphic sequence (S3) with a parting (P) removed and analyzed separately.

\begin{tabular}{|c|c|c|c|}
\hline Sample ID & Strat & Thickness (feet) & Explanation \\
\hline DA2169254 & D169254-00A:01,02,04-S3 & 8.3 & $\begin{array}{l}\text { Upper Level sample }(-00) \text { mathematical composite obtained } \\
\text { by combining the upper and lower sections of a single } \\
\text { sample containing a parting. }\end{array}$ \\
\hline D169254 & D169254-01-S3 & 5.5 & 1 st coal bench $(01)$. \\
\hline D169253 & D169254-02,04-S3 & 2.8 & $\begin{array}{l}\text { 2nd coal bench (combination of stratigraphic levels } 02 \text { and } \\
04 \text { ) without the parting (03P). }\end{array}$ \\
\hline
\end{tabular}




\section{Appendix 7. Identification of Nearby Samples}

Georeferencing, used to identify samples that are located within one mile of another sample's location, is computed by the database using the following equation based on the law of spherical cosines (Veness, 2010):

$$
\mathrm{D}=\operatorname{acos}\left[\sin \left(\mathrm{lat}_{1}\right) \times \sin \left(\mathrm{lat}_{2}\right)+\cos \left(\mathrm{lat}_{1}\right) \times \cos \left(\mathrm{lat}_{2}\right) \times \cos \left(\operatorname{long}_{2}-\operatorname{long}_{1}\right)\right] \times \mathrm{R}
$$

where:

$\mathrm{D} \quad$ is distance in miles between two geographical points

$\mathrm{R} \quad$ is mean radius of earth, equal to 3,959 miles

lat $_{1} \quad$ is latitude of sample 1

$\operatorname{long}_{1}$ is longitude of sample 1

lat $_{2}$ is latitude of sample 2

$\operatorname{long}_{2}$ is longitude of sample 2 


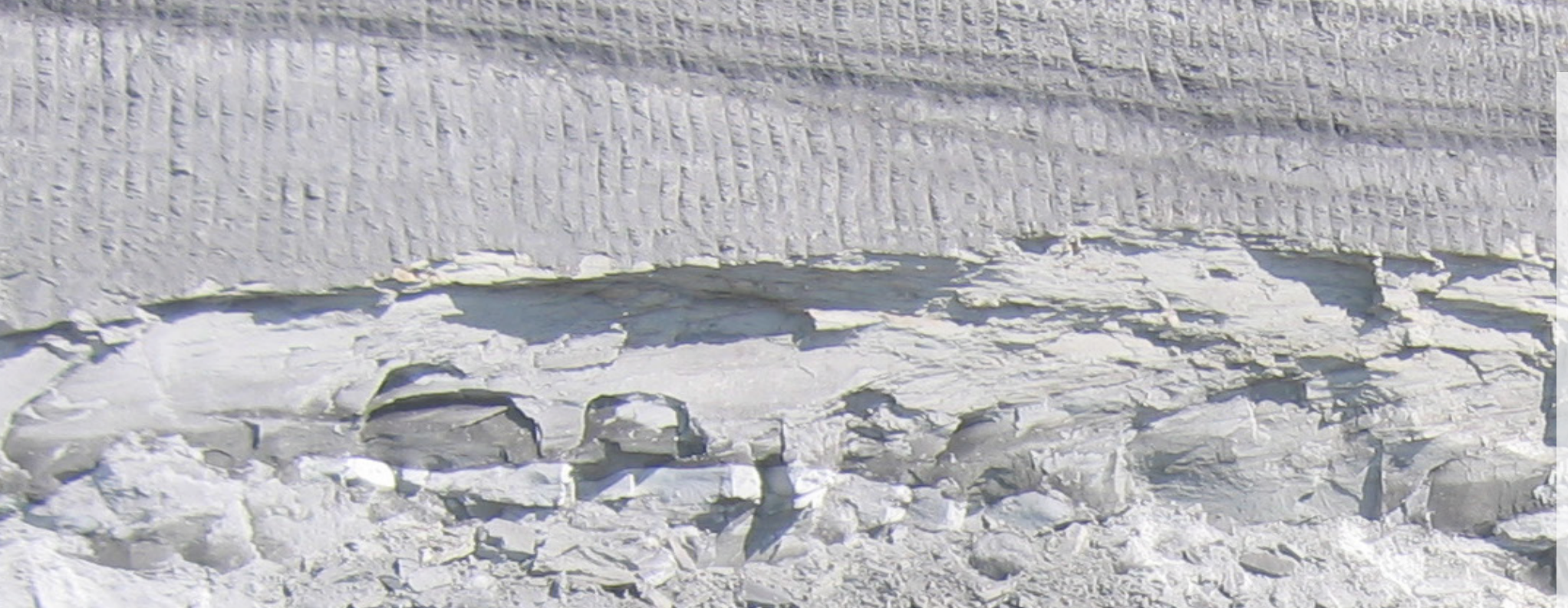

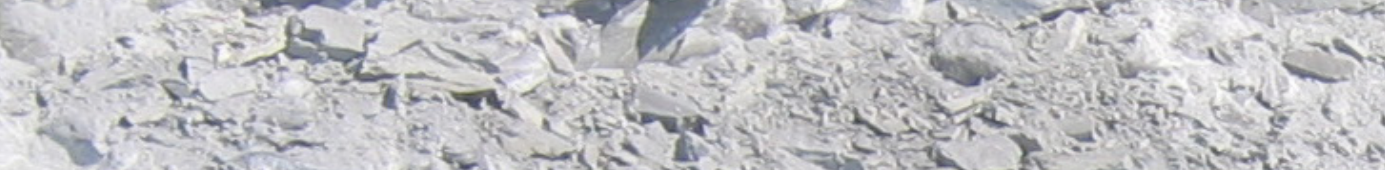

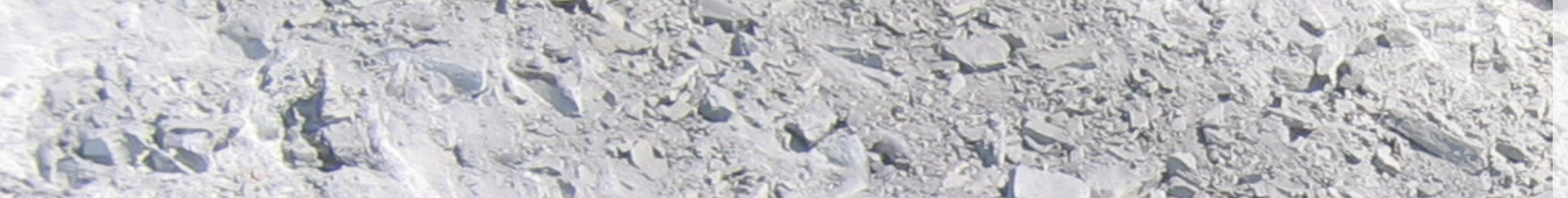

